\title{
Near Death Experiences: Falling Down a Very Deep Well
}

\author{
Claudio Messori \\ Str. Villaggio Prinzera 1, Fraz. Boschi di Bardone, Terenzo, Italy \\ Email: messori.claudio@gmail.com
}

How to cite this paper: Messori, C. (2018) Near Death Experiences: Falling Down a Very Deep Well. Open Access Library Journal, 5: e4985.

https://doi.org/10.4236/oalib.1104985

Received: October 15, 2018

Accepted: October 30, 2018

Published: November 2, 2018

Copyright $\odot 2018$ by author and Open Access Library Inc.

This work is licensed under the Creative Commons Attribution International License (CC BY 4.0).

http://creativecommons.org/licenses/by/4.0/

(c) (i) Open Access

\begin{abstract}
The huge progress made in recent decades in the medical-health field, together with the large-scale introduction of cardiopulmonary resuscitation (CPR) and other emergency techniques and procedures, has made it possible to save a growing number of human lives. Also because of this, the testimonies classified as Near Death Experiences (NDEs) have increased exponentially, mostly in Western countries. The present work aims at first to identify six basic inclusion-exclusion clinical criteria to be adopted for the definition of a Near Death State (NDS), distinguishing between an objective NDS and a subjective Near Death Like State (NDLS). This classification leads to having NDEs split into two distinct groups: Near Death Experiences associated to NDS and Near Death Like Experiences (NDLEs) associated to NDLS. A general frame of reference, namely Tenso-Relational Model, within which consciousness and the mind-body system (MBS) can be located, is discussed. In conclusion it is introduced a bio-physical frame of reference to be adopted for a possible explanation 1) of the so called Out of Body Experience (OBE), which can occur while experiencing an NDS or an NDLS, 2) of the so-called Electromagnetic After-Effects (EAEs), namely electro-sensitivity, which can be caused both by an NDS or by an NDLS, and 3) of other NDS-NDLS aftereffects such as the so-called Energy Healing Abilities (EHA) and the psi abilities, i.e. psychokinesis, telekinesis and psychic-teleportation, and Extra-Sensory Perceptions (ESPs) such as telepathy, clairvoyance, precognition and psychometry.
\end{abstract}

\section{Subject Areas}

Anthropology

\section{Keywords}

Near Death State vs Near Death Like State, Near Death Experience vs Near 
Death Like Experience, Tenso-Relational Model, Mind-Body System, Out of Body Experience, Electromagnetic After-Effect, Psi phenomena, ESP

\section{Introduction}

The finger pointing at the moon, is not the moon ${ }^{1}$.

The expression Near Death Experience (NDE) ${ }^{2}$ [1] was coined in 1975 by American psychiatrist and philosopher Raymond Moody Jr., a lover of regressive hypnosis, to indicate a particular organic, cognitive, perceptive, psychological, spiritual state of which an individual near or during the process of dying can experience, a state to which in 1892 the Swiss geologist Albert von St. Gallen Heim had already dedicated a collection of testimonies, issued by precipitated climbers and survivors during high mountain climbing (Alps), by soldiers seriously injured in war, by workers fallen from scaffolding, and by people who risked dying from drowning or following an accident.

Every instant of life is, strictly speaking, a fleeting instant which is a prelude to death, and every experience lived in that fleeting instant, it can be seen as a pre-mortem or near-death experience. However, when Raymond Moody Jr. coined the expression Near Death Experience, he referred to some particular pre-mortem or near-death experiences, and not to any experience at any instant of life. Since then (1975), the use of the term NDE has become part of the scientific vocabulary, as well as of the common one, without there being a shared opinion on what should be understood by pre-mortem or Near-Death State (NDS).

If we do not have a shared definition of what should be meant by NDS, the investigation and discussion related to NDE can easily run out in considerations like " $A$ person is where he believes himself to be; or, more technically, the body is an object in the field ofthe self (...) In other words, the human animal can be symbolically located wherever he feels a part of him really exists or belongs" [2], or fall down into a very deep well of experiences which are doing a great job in Alice's Wonderland, but that turns out to be quite useless as evidence for, e.g., the existence of a dimension populated by ethereal beings (including the de" "Bhikkhus, the teaching is merely a vehicle to describe the truth. Don' $t$ mistake it for the truth itself. A finger pointing at the moon is not the moon. The finger is needed to know where to look for the moon, but if you mistake the finger for the moon itself, you will never knowthe real moon. The teaching is like a raft that carries you to the other shore. The raft is needed, but the raft is not the other shore. An intelligent person would not carry the raft around on his head after making it across to the other shore. Bhikkhus, my teaching is the raft which can help you cross to the other shore beyond birth and death. Use the raft to cross to the other shore, but don't hang onto it as your property. Do not become caught in the teaching. You must be able to let it go." (Thich Nhat Hanh, Old Path White Clouds: Walking in the Footsteps of the Buddha).

${ }^{2}$ About the subject see e.g.:

- UVA School of Medicine, Department of Psychiatry and Neurobehavioral Sciences, Division of Perceptual Studies, Academic Publications

https://med.virginia.edu/perceptual-studies/publications/

- International Association for Near-Death Studies, Inc.:

http://iands.org/resources/recommended-reading.html 
ceased) parallel to the ordinary one we experience daily.

Starting from the last decades of the twentieth century, the diffusion and progressive improvement of cardiopulmonary resuscitation (CPR) techniques, together with an overall improvement in medical-scientific knowledge and know-how, has allowed to save a growing number of human lives $^{3}$ [3], followed by a significant increase in the testimonies related to particular states [4], experienced near or during the process of dying (pre-mortem). The over-all incidence-reports of NDEs in literature have varied widely from a modest Figure of $10 \%$ to around $35 \%$, even up to an incredible Figure of $72 \%$ in persons who have faced close brush with death [5].

A phenomenon that, due to lack of adequate inclusion/exclusion criteria, has frequently given rise to self-referential and scarcely reliable reports.

The purpose of this work is not to report or analyze the many testimonies cataloged as Near Death Experiences, namely the subjective experience occurring during an objective Near Death State (NDS) or during a subjective Near Death Like State (NDLS), not even to provide novel neurological data to be adopted in explaining the non-ordinary sensory and cognitive conditions that could be experienced during an NDS or an NDLS [7], nor to validate a NDE by completing an alternative, or integrative, scale to the existing ones, such as the Greyson's Near Death ExperienceScale (NDES) [8] [9].

This work is based on the assumption that consciousness and mind-body dynamics deserve to be investigated also in relation to non-ordinary or altered or border-line states such as those that can characterize an NDS or an NDLS, regardless of narratives that can be given in this regard. For this purpose, the first step to accomplish is to try to clarify why and how an NDE, namely the subjective retrospective report of a person's experience during a NDS or a NDLS (which may include 1) visual, auditory, kinesthetic, olfactory perception, whose relevance to the context and dynamics of the event it may or may not be confirmed by eyewitnesses, and 2) some not ordinary sensory and cognitive processes, suggesting the possibility of mind to function independent of the physical body), should be selected on the basis of the neuro-psycho-physiological state in which the Near Death Experiencer (NDEr) is or was on the occasion of his/her reversibly death (the irreversibly dead have not shown, so far, any willingness to release interviews, at least not to curious, open minded but ordinary people like me).

The availability or non-availability of the clinical information necessary to es-

${ }^{3}$ Until the end of the last century, the percentage of people who thanks to CPR and advanced cardiac support (ACLS) survived an out-of-hospital cardiac arrest, was very low, and among those who did survive, the percentage of those who did not report major neurological damage it was also very low. As Stefan Timmermans reports [3]: “(...) The number of people resuscitated from sudden death by emergency personnel is not known. Nor is it known how many people can be resuscitated with a reasonable chance of surviving and remaining neurologically intact... Thought no national averages are available on the proportion of people who survive out-of-hospital cardiac arrests, current estimates suggest that no more than $1 \%-3 \%$ of victims live to be discharged from the hospital. The true percentage is probably even less." These low percentages mean that in the overwhelming majority of resuscitative efforts, people die. 
tablish the neuro-psycho-physiological state of the subject, constitutes the first inclusion/exclusion criterion to be adopted in the compilation of a reasoned classification of the NDE testimonies.

Accordingly, thepresent work is aimed at:

1) Defining (Section 1) which basic clinical criterion, and which general criteria of inclusion/exclusion, can be adopted to distinguish between a Near-Death State (NDS), i.e. a neuro-psycho-physiological state experienced near or during the temporary and reversible objective ending stage of the process of dying, during which the subject is judged to be clinically dead, and a similar but not equivalent state, namely a Near-Death Like State (NDLS), that is a neuro-psycho-physiological state experienced near or during a temporary and reversible subjective premortem-like state (e.g. apparent death), far from a clinically established reversible ending stage of the process of dying (no clinical death).

2) Providing a general frame of reference within which consciousness and the mind-body system (MBS) can be located (Section 2), introducing the discussion over their explanation in terms different from those adopted in the debate on consciousness and MBS started off and developed mostly in the United States, where the philosophy of the dominant science-philosophy of mind-is a philosophy of an analytic type, which is essentially concerned with giving good definitions for categories and objects. In doing so I'm following Francisco Varela's point of view on the subject, clearly expressed while interviewed by Sergio Benvenuto in 2002 on the topic of Consciousness in the Neurosciences [10]:

There is a reductionist tendency in accordance with which the notion of NCC (Neuronal Correlates of Consciousness) truly occupies the majority of debates, but some of us-evidently I am not alone, even though we are still a minority-think that the question posed in these terms has no solution, for the simple reason that lived experience as such is in principle logically and empirically non-reducible to a neuronal function. This is what is called the hard problem of consciousness. That which belongs to the realm of lived experience has a stature or a nature that cannot be explained in terms of the neuronal system. A correlate can be found, but this correlate absolutely does not change the fact that the phenomenal aspect remains a phenomenal apparition, a phenomenal accession to my consciousness. It is thus necessary to present the discussion in different terms.

3) Suggesting a bio-physical frame of reference (Section 3) to be adopted for a possible explanation of the so called Out of Body Experience (OBE) [11], that can occur while experiencing an NDS or a NDLS, and of the so-called Electromagnetic After-Effects (EAEs), namely electro-sensitivity, that can be caused by an NDS or by an NDLS.

4) Suggesting abio-physical frame of reference (Section 4) to be adopted for a possible explanation of others NDS-NDLS aftereffects such as the so-called Energy Healing Abilities (EHA) and the so-called psi phenomena, i.e. psychokinesis, telekinesis and psychic-teleportation, and Extra-Sensory Perceptions (ESPs) 
such as telepathy, clairvoyance, precognition and psychometry, which interweaving with the narration of and on the NDEs and NDLEs do not fail to make the paranormal halo that surrounds them even more appealing.

What are then the neuro-psycho-physiological peculiarities that distinguish an NDS, to which an NDE can legitimately refer, from an NDLS, to which not an NDE but a Near Death Like Experience (NDLE) can legitimately refer, especially when a typical or altered or other-than-ordinary states of awareness and consciousness are at stake?

\section{Near Death State vs Near Death Like State: Basic Distinctive Criteria}

The subjective nature and absence of a frame of reference for this experience lead to individual, cultural, and religious factors determining the vocabulary used to describe and interpret the experience. Pim Van Lommel [12].

Based on the detection of the four plus one primary vital signs (life-sustaining: body temperature, $\mathrm{BT}$, blood pressure, $\mathrm{BP}$, heart rate, $\mathrm{HR}$, respiratory rate, $\mathrm{RR}$ and oxygen saturation, OS), the neuro-psycho-physiological state of the subject experiencing (and at a variable later time reporting) a pre-mortem state can be traced to two distinct categories:

1) A neuro-psycho-physiological state experienced near or during the temporary and reversible objective ending stage of the process of dying, called Near Death State (NDS), during which the life-sustaining signs are shown to be absent and the subject is judged to be clinically dead (clinical death is still a reversible condition, which is to be distinguished from the irreversible and definitive cellular death or cell apoptosis of all single cells constituting the organism, which occurs as a progressive breakdown of metabolic processes, resulting in irreversible deterioration of the affected systems and organs of the body) [13] [14], that is: severe hypothermia (body temperature below 70 degrees Fahrenheit, 21 degrees Celsius ${ }^{4}$ ), no pulse, no breathing, no oxygen pressure, no blood pressure. At the onset of clinical death, consciousness is lost within several seconds or minutes [15] and measurable brain activity stops within 20 to 40 seconds. Since both consciousness and awareness do not follow the law of all or nothing, i.e. there may be different degrees of consciousness and awareness, the dissociation (disconnection) state elapsing between the bodily (energy) and mental (tension) dimension eventually occurring during a NDS, may induce the subject to experience an extra-sensory, floating, disembodied and disembedded state of detached awareness (a psychic-tension regime disengaged from the Tension-Phase Cor-

${ }^{4}$ The medical record reports at least three cases of people of different ages, judged clinically dead due to a profoundly hypothermia, body temperature below $70^{\circ} \mathrm{F}$, who thanks to medical care and to CPR have survived with an intact neurological outcome. They are: American Michelle Funk, at the time (1986) 30-months-old, resuscitated after her body temperature had lasted for more than an hour at $66^{\circ} \mathrm{F}\left(19^{\circ} \mathrm{C}\right)$; Norwegian Anna Bagenholm, at the time (1999) 29-years-old, resuscitated after her body temperature had lasted for more than an hour at $56.7^{\circ} \mathrm{F}\left(13.7^{\circ} \mathrm{C}\right)$; Swedish Stella Berndtsson, at the time (2011) 7-years-old, resuscitated after her body temperature had lasted for more than an hour at $55.7^{\circ} \mathrm{F}\left(13.2^{\circ} \mathrm{C}\right)$. 
relative Dynamics, see Section 2 for explanation, often mistakenly confused with astate of detached consciousness, that may occur during a self-induced NDLS, see Section 3 for explanation), i.e. a submerged mental state in which all ordinary cognitive functions are zeroed while the subject's egoic psychic complex, or self or center of awareness (to be distinguished from consciousness, see Section 3), namely “(...) an enduring entity (i.e. the feeling that we are the same person across time) to which certain mental events and actions are ascribed (i.e. the feeling that we are the authors of our thoughts and actions) and which is distinct from the environment" [16], is later on recalled as if it has been (subjectively) experienced somehow detached from the body and/or from the environment.

This state of (temporary and reversible) detachment of the subject's egoic psychic complex (detached awareness) may allow the subject to entertain a devoid of space-time coordinates, passive relationship with her/his inner-insight (embodied) and/or with the outer-outsight (embedded) [17] (Figure 1).

Such experience it occurs while the mind-body system activity it is suspended within the Tension-Tension Correlative Dynamics (involving domains of tension coupled to the subject's psychic $\rightarrow$ mentaP inner-insight and domains of tension coupled to the subject's outer-outsight, see Section 2 and 3 for explanation) elapsing between the EM sensory module and the subject's egoic psychic complex (see Section 3 for explanation).

2) A neuro-psycho-physiological state called Near Death Like State (NDLS) ${ }^{6}$, experienced near or during a temporary and reversible subjective premortem-like state, during which the life-sustaining signs are shown to be eventually ${ }^{5}$ As I discussed in [18], the psychodynamic distinction that can be made between psychic function, mental function, cognitive function, is the following:

- The psychic function is the relational capacity, distinctive, involved in the phylogenetically prescribed arrangement of the human psychic environment (insight), i.e. its species-specific tensoriai basin of attraction (the particular tensorial warp on which it can be weaved the energy weft characterizing the genus Homo) in whom are dissolved all the phase transitions (bifurcations) who went to meet the psychism (C.G. Jung) of the biological phenomenon in the course of phylogeny, passing through the neurological transition up to the bifurcation that led to the emergence of the genus Homo.

- The mental function is the explicated, modal and procedural, form of the psychic function. It refers to the process of in-formation who went to meet the human psychic environment in the relationship with the surrounding.

- De facto, the psychic function and the mental function have always been compresent (what varies is the prevalence of one over the other), pointingat the Jungian concept of archetype (psychic raw material) and to that of collective unconscious.

- From the psycho-dynamic point of view, these two functions qualify the constitutive arrangement, and dynamics, of the psychic $\rightarrow$ mental environment which precedes the psychological birth of the genus Homo (Middle Paleolithic).

- The cognitive function, is the relational capacity, gained from the particular differentiation, and subsequent arrangement, of the psychic $\rightarrow$ mental environment following the psychological birth and, in particular, the development of oral language together with the maturation of cognitive thinking ( $\rightarrow$ bipolar psychic dimension thinking-feeling $\rightarrow$ logical-abstract thinking), which may be understood as the ability to program significant actions with respect to the ability to formulate meaningful purposes, by resorting to the exercise of ideation and ratiocinative discrimination.

${ }^{6}$ See e.g.: International Association for Near-Death Studies, Inc., Near-Death-Like Experience, http://iands.org/nde-stories/nde-like-accounts.html 


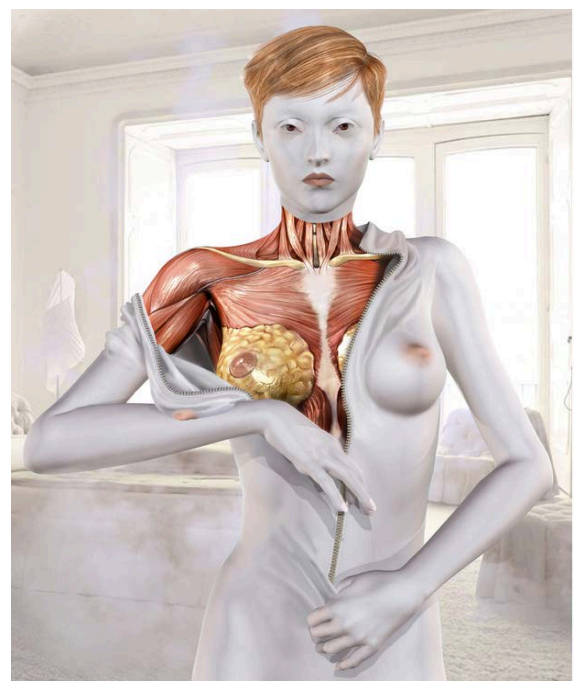

Figure 1. Gianluca Traina: Human Default-Skin Dress of human body. As individuals we experience ourselves as biologically discrete, as contained within our skins. Thus, we experience ourselves as embodied and largely define our selves and our boundaries by our bodied experience. But also importantly, despite the ecological reality of our inextricable embeddedness, we are boudaried, defined, and located by others-and, so, we are also embodied by others [6]. Image source:

https://gianlucatraina.com/portrait-360/human-default/.

deeply altered but still present (no clinical death), characterized by a submerged cognitive state in which all ordinary cognitive functions are NOT zeroed but deeply depressed or altered, which may present some aspects in common with a NDS, such as a state similar but not equivalent to the state of detached awareness, and some of the experiences reported by subjects who have experienced the latter (such as Out of Body Experience or OBE; malaise or well-being feelings, sometimes accompanied by the vision of a light at the end of a tunnel and of deceased loved ones or other intangible entities; visual recapitulation of one's own life $^{7}$ ) [19], regardless of age, gender, cultural, religious or social background.

The distinction between NDS group (where the subject is judged to be clinically dead on clinical evidence via life-sustaining criteria) and NDLS group (no clinical evidence for clinical death), it should be then given by the presence or absence of the following clinical signs, whose presence has an inclusion value for NDS:

- arrest of cardiac rate and pulmonary ventilation rate (no pulse, no breathing, increasing hypothermia);

- isoelectric electroencephalogram, i.e. flat EEG;

- cerebral anoxia (no oxygen pressure, no blood pressure);

${ }^{7}$ According to SHI TRO [20], i.e. the Bon Peaceful \& Wrathful (Shi-Tro) Deities (tibetan: Shitro Lhatsog [zhi khro lha tshogs]), this experience is experienced during the fourth phase (the wind dissolves in space) of the five phases that make up the process of dying (translation from Italian is mine): (...) You can experience a quick vision that shows the habits and emotional patterns that have characterized the life you are leaving. (...) Very vivid memories appear momentarily and then disappear before reappearing in new combinations. It's a bit like reviewing the news on a newscast. The nature of one's habit patterns is clearly shown at this stage. 
- absence of the pupillary reflex and whole-body areflexia;

- zeroed (temporary and reversible) state of consciousness, i.e. temporary and reversible zeroing of the physiological psycho-perceptive and psycho-relational activity, where the emptiness left by the quasi-complete lack in awareness of oneself and one's actions is filled byan adrift-ungrounded self (see Section 3 for explanation).

The absence of detection, or the inability to dispose of one or more of the aforementioned clinic signs, does not allow the verification of the presence or absence of clinical death, a sufficient reason not to include the subject in the NDS group but in the NDLS group.

Accordingly, when approaching NDEs testimonies, the first inclusion/exclusion criterion to be adopted to establish their value, is to distinguish between neuro-psycho-physiological states experienced near or during the temporary and reversible objective final stage of the process of dying (NDS, clinical death), which usually requires intensive care intervention, i.e. resuscitative efforts, and, on the other hand, neuro-psycho-physiological states experienced near or during a temporary and reversible subjective premortem-like state (NDLS, no clinical death, although resuscitation efforts may be required), far from a clinically established final but temporary and reversible stage of the process of dying.

If we adopt this basic inclusion/exclusion criterion, it arises the reasonable possibility that among the countless testimonies classified as NDE, only a little part may legitimately fit into the NDS group, while the majority of them may belong to the NDLS group, therefore classifiable as NDLEs.

\subsection{Near Death State's Group}

Ji Lu asked about serving the spirits. The Master said, "While you are yet not able to serve men, how could you be able to serve the spirits?" "May I ask about death?" "When you do not yet understand life, how could you understand death?" Confucius ${ }^{8}$

Possible causes that can induce an NDS (clinical death) include: cardiac arrest, shock after loss of blood, coma following traumatic brain injury or intra-cerebral hemorrhage, near-drowning or asphyxia, septic or anaphylactic shock, electrocution, attempted suicide, but also serious diseases not immediately life-threatening [12] [21].

Statistically, the maximum time within which an individual judged clinically dead may return to life (generally following the favorable outcome of medical care and/or CPR resuscitation; after cardiac arrest, e.g., for every minute without CPR, the chance of death increases by 10 percent [22]), with or without assisted ventilation $(\mathrm{AV})$, without reporting irreversible or partially reversible brain damage, due to cerebral anoxia, does not exceed 2 to 4 minutes, after which, in the absence of a heartbeat recovery and/or of AV, the risk of irreversible or partially reversible brain damage increases exponentially with the passing of mi${ }^{8}$ The Analects of Confucius http://www.indiana.edu/ p374/Analects_of_Confucius_(Eno-2015).pdf 
nutes.

As a consequence, the subjects to date interviewed for NDE's testimony, to be reliable, must have not reported irreversible or partially reversible brain damage, no permanent organic impairment of the neuropsychological and/or psychic functions. If they did, their NDE's testimony would be unreliable and lapses. Moreover, resorting or not resorting to AV implies a different average time of latency of the state of (temporary, reversible) unconsciousness (an average duration ranging from 2 to 4 minutes without $\mathrm{AV}$, longer with $\mathrm{AV}$ ). Therefore this aspect constitutes the first inclusion/exclusion criterion to be adopted in the distinction among the NDS group: NDS-WAV (without AV) vs NDS-UAV (under AV).

Furthermore, we must distinguish between NDS pharmacologically untreated or treated, e.g. with anesthetic or with epinephrine injection in shocked patient or with drug-induced coma in head injury. Resorting to pharmacological treatment may have had a psychotropic action, in which case the NDE's testimony may not be considered reliable.

This is the second inclusion/exclusion criterion to be adopted in the distinction among the NDS group: NDS-UMIPE (Under Medically Induced Psychotropic Effect) vs NDS-WMIPE (Without Medically Induced Psychotropic Effect). This same inclusion/exclusion criterion it has to be applied also among the NDLS group, distinguishing between subjects who have experienced a pre-mortem status under medically or self-induced psychotropic effect (NDLS-UPE, that is Under Psychotropic Effect) and subjects who have experienced a pre-mortem status without medically or self-induced psychotropic effect (NDLS-WPE, that is Without Psychotropic Effect).

\subsection{Near Death Like State's Group}

Among the NDLS group we find cases of apparent death associated to catalepsy, to states of deep sleep, mystical ecstasy, deep meditation, hypnotic states-this is the case, e.g., of lethargy and catalepsy as classified by neurologist Jean-Martin Charcot (1825-1893) [23] - , cerebral hypoxia, as the one observed in pilots of supersonic aircraft, altered states of consciousness induced by the intake of psychotropic substances, as in psychedelic experience, or by pulmonary hyperventilation, as in the circular breathing (holotropic respiration) adopted e.g. in transpersonal rebirthing, and, last but not least, tanatosis or simulated death.

Among all these states, in the course of which the subject may manifest an apparent state of death, are the state of catalepsy and the state of tanatosis (both may last for hours, days or even weeks) to deserve here a brief discussion.

Apparent death associated to catalepsy (psycho-neurotic, psycho-organic, induced or self-hypnotic, voluntary, mediumistic, shamanic, narcoleptic), has some elements in common with the tanatosis or simulated death (see below), and is characterized by a deep state of depression, or lethargic dulling, affecting one or more, and in varying degrees, of the following functions: 
- motor functions (striated muscles akinesia or hypokinesia, hypo- or hypertone, areflexia or hyporeflexia),

- vegetative functions (bradycardia, pulmonary hypoventilation, smooth muscle hypokinesia),

- sensory functions (visual, auditory, kinesthetic, gustatory, tactile, olfactory anesthesia or hypoesthesia),

- attentional functions (subliminal level of the state of vigilance activation or arousal or tonic alert),

- cognitive functions (altered state of the relatively independent psycho-physical relational modules), whose co-action and inter-action qualifies a state of consciousness, which it allow the subject to interact with the environment, to modify it and to modify (feed-forward viapsycho-phisycal processes) its own adaptive behavior, according to a tendency to overcome behavioral stereotypy.

Apparent death associated to tanatosis, or simulated death (called death feigning by Charles Darwin, which is commonly observed in ethology, were is adopted for defensive purposes, both by invertebrates such as beetles and arthropods, and by vertebrates such as birds, mammals, reptiles, amphibians and fish), may determine in the subject an experience of tanatomimesis, that is Thanato Mimesis Experience (TME) [24], during which the subject may show one or more of the following clinical signs:

- intensively and extensively variable (transitory and reversible) states of spatio-temporal alteration or dilatation of the mind-body correlative dynamics (e.g. during hypnotic transe, mystical ecstasy, mediumistic transe, shamanic transe, states of possession, OBEs, abductions, deep meditation), together with alteration or inhibition of physiological psycho-perceptive and psycho-relational activity (e.g. hallucinatory states ${ }^{9}$, such as hallucinations from sensory deprivation, hypnagogic and hypnopompic, psychedelic, psychiatric), interpretable as intensively and extensively variable (transitory and reversible) states of spatio-temporal alteration or dilatation elapsing between Phase Conjugate Dynamics and Tension Conjugate Dynamics (see Section 2 and 3);

- voluntary reduction or increase of blood flow,

- low voltage electroencephalogram with a spectrum of mixed frequencies with prevalence of Delta waves, EEG type of sleep at the REM stage,

- high voltage electroencephalogram with coherent spectrum of frequencies with prevalence of Gamma, Theta and Alpha waves, EEG type of deep meditative state [25] [26].

\subsection{NDS and NDLS Final Remarks}

(...) and burning with curiosity, she ran across the field after it, and fortunately

${ }^{9}$ According to the American Psychiatric Association a hallucination is defined as a "sensory perception that has the compelling sense of reality of a true perception but that occurs without external stimulation of the relevant sensory organ" (1994, p. 767). 
was just in time to see it pop down a large rabbit-hole under the hedge. The rabbit-hole went straight on like a tunnel for some way, and then dipped suddenly down, so suddenly that Alice had not a moment to think about stopping herself before she found herself falling down a very deep well. Lewis Carrol ${ }^{10}$ (1865)

Summarizing what stated so far, before making any assessment, formulating hypotheses, drawing conclusions, expressing opinions on what reported by those that are indistinctly referred to as NDErs, it is necessary to carry out a classification about the NDEs based on the clinical conditions of the NDEr during his/her objective (NDS) or subjective (NDLS) pre-mortem experience.

The basic inclusion/exclusion criteria that can be introduced to perform this preliminary classification are just six:

1) Availability or non-availability of the clinical information necessary to establish the neuro-psycho-physiological state of the subject, i.e. clinical death vs no-clinical death. Non-availability of clinical data is sufficient reason to classify the reported experience among the NDLEs group associated to NDLS.

2) Status of pre-mortem, i.e. clinical death vs no-clinical death, established on the basis of the presence or absence (of one or more) of the life sustaining clinical signs. The absence of one or more of the life sustaining clinical signs is a sufficient reason not to include the subject in the NDS group but in the NDLS group.

3) Subjects must have not reported irreversible or partially reversible brain damage, no permanent organic impairment of the neuropsychological and/or psychic functions. If they did, their NDE's testimony would be unreliable and lapses.

4) Among the NDS group it should be distinguished between subjects who have experienced a pre-mortem status under AV or without AV. This is a first inclusion/exclusion criterion which divides the NDS group into two subgroups: NDS-UAV vs NDS-WAV.

5) Among the NDS group it should be distinguished between subjects who have experienced a pre-mortem status under medically induced psychotropic effect or without medically induced psychotropic effect. This is asecond inclusion/exclusion criterion which divides the NDS group into two subgroups: NDS-UMIPE vs NDS-WMIPE.

6) The latterinclusion/exclusion criterion it has to be applied also among the NDLS group, distinguishing between subjects who have experienced a pre-mortem status under medically or self-induced psychotropic effect (NDLS-UPE) and subjects who have experienced a pre-mortem status without medically or self-induced psychotropic effect (NDLS-WPE).

Despite planetary spread of CPR, exponential increase in NDE, NDLE and TME's testimonies is an eminently late $20^{\text {th }}$ early $21^{\text {th }}$ century Western phenomenon, with few exceptions [27] [28].

${ }^{10}$ Charles Lutwidge Dodgson, Alice's Adventures in Wonderland, Chapter I, Down the Rabbit-Hole https://www.cs.cmu.edu/ rgs/alice-I.html 
In the background, it stays a hyper-techno-centric society committed to removing death and dying from its perspective, condemned to the pursuit of a chimeric immortality, in the Promised Land of Science's Rainbow Body.

The current post-materialist but still deeply positivist perspective of knowledge, with its transposition of the cult of the Universal Spirit (intentional energy and absolute will) into the cult of Universal Consciousness ${ }^{11}$ [29], seems to be moving in the direction indicated by a carbon-fiber finger pointing at a $3 \mathrm{D}$ printed mandala-moon: the promises of future scientific developments that will allow to tame and control, in whole or in part, our vulnerability, caducity and impermanence.The Promised Land of Immortality.

A phenomenon that, also with complicity of globalized communication and cultural exchanges, re-proposes the millennial theme of death, dying and post-mortem, calling into question, among others, the Christological eschatology (from the Greek etymon "[éskhatos] last and [loghìa] speech", i.e. "science of the last things"), the Western philosophical tradition, the Eastern metaphysical tradition, as well as a number of magical and mysterious beliefs and practices traceable in different times and places (up to the Western $20^{\text {th }}$ century occult revival [30]).

As Bruce Greyson observes [31]:

Adherents to various religions have claimed NDEs as empirical support of their particular doctrines. It has been argued that NDEs provide striking parallels to the teachings of the Hindu Upanishads and to early Babylonian, Egyptian, and Zoroastrian texts (Holck 1978-79), to shamanism (Green 2001), to Taoism (Hermann 1990), to Sino-Japanese Pure Land Buddhism (Becker 1981), to Tibetan Buddhism (Becker 1985; Holck 1978-79), to Gnostic Christianity of the second through fourth centuries (Bain 1999), to medieval Christian religious treatises (Zaleski 1987), to Mormon doctrine (Lundahl 1983), to Christian Universalism (Vincent 2003), to Bishop John Shelby Spong's New Christianity (Gibbs 2005), and to New Age spirituality (Lee 2003). Regarding these "religious wars" over NDEs, Ring expresses regret that "the body of the NDE, like some sort of Bruce Greyson 407 sacred relic or corrupted corpse, is fought over by warring parties either for rights of possession or unceremonious burial' (2000, 240). Ring found beliefs common among NDErs that seemed consistent with a Judaeo-Christian worldview (1980b). He reports that more than half of his sample of NDErs reached a point where a decision was made either by or for them as to whether they would return to physical life, and 40 percent of them described an encounter with a presence (not always seen) that included an auditory or telepathic conversation. Sometimes the presence would describe specific consequences of the decision whether or not to return, including information about

${ }^{11}$ Positivism is not new to operations like this. In the last years of his life, Auguste Comte (1798-1857), the ideologist of positivism, wrote the Positivist Catechism and founded the Positivist Church, where the doctrinal, ethical and liturgical elements of the Catholic tradition are transposed. The novelty lies in the fact that today, the Neo-Positivist Catechism with its Neo-positivist Church and its cult of Universal Consciousness, transposes the doctrinal, ethical and liturgical elements of other religious and cultic traditions, as well as the Catholic one. 
what would occur if the experiencer chose to return to life. This often was accompanied by a life review, and sometime a preview of events to come, in which the experiencer evaluated his or her life. Most experiencers interpret this sequence of events as a direct encounter with God and with God s unconditional love. Ring himself interprets this sequence of events as an encounter not with an external deity but rather with the experiencer's "higher self", of which the individual personality is only a fragment. This higher self seems to have divine aspects and is clearly omniscient with respect to the personality, Ring speculates that experiences of this encounter may give rise to the Christian concept of the guardian angel. Ring later documents further psychological effects in NDErs evidencing changes in attitude and belief that are consistent with the dominant Western Judaeo-Christian heritage (Ring and Valarino 1998).

The newly born secular religion is thus added to other religions and cultic traditions, each of them with some astonishing anecdotes to tell and their own interpretations on the subject. The Dzogchen ${ }^{12}$ Tradition's testimonies, concerning the non-natural disappearance (dematerialization-like, which most often presents as complete self-induced body dehydration or mummification) of the body of advanced Dzogchen practitioners, belongs to one of these [32] (translation from Italian is mine):

At death, the greatest Dzogchen practitioners (...) leave the body (...) and manifest the rainbow body. (....) Others leave the body in a flash of lightor in a pile of flames (on the subject see e.g. [33] [34] [35] [36]).

Sometimes the light is white, sometimes blue or green. Occasionally a white, yellow, red, blue and green light appears. These lights remain for a while and then fade away. The flames are visible from those present, but when the combustion ends, there remains no trace of the body, neither the bones, nor a bit of ashes. simply everything has dissolved. (...) The third category of realized practitioners (...) when they leave the body also join the brightness of true nature. Externally one can see light, water or other elements dissolving (...). The fourth ${ }^{12}$ The Tibetan word Dzogchen indicates a teaching belonging to the system of teachings of Tibetan Vajrayana Buddhism and to the pre-Buddhist Bon religion, a central teaching of the Tibetan Buddhist School Nyingmapa. The Sanskrit word for Dzogchen is Ati Yoga or Primordial Yoga. According to the Tibetan tradition, the Dzogchen teaching was introduced by the Radice Master Garab Dorje (1st century AD?) in Oddyana, an ancient region that many identify with the present Swat District in the North-West of Pakistan. According to the Bon tradition, however, the Dzogchen was introduced by Master Shenrab Miwoche (2nd century BC?) in Zhang Zhung, an ancient Himalayan region that was annexed to Tibet during the seventh century A.D. Historically and geographically, the debut of the Dzogchen teaching would thus be placed in the period of transition between the Maurya Empire (320-185 BC) and the Kushan Empire ( $1^{\text {st }}-3$ rd century AD). The Dzogchen, or Great Perfection, indicates the natural and primordial state, that is the spontaneous condition of the mind, and, at the same time, the corpus of teachings aimed at realizing this condition. Our ultimate nature is defined as pure and all-encompassing, as a primordial awareness and always naturally present as the background of every psychic event. This intrinsic awareness has no form of its own and yet makes perception possible as well as allowing every sentient being to experience, reflect or express themselves in all forms. The analogy provided by the masters of the Dzogchen tradition is that the nature of a living being is like a mirror that reflects objects in a total and comprehensive way, but that in no case is it ever influenced by the same reflections; or as a perfectly transparent crystal sphere, able to take in itself the color of light to which it is exposed, without changing its original nature, neutral and devoid of its own color. 
type of death is known as the way of the dakini transformations. As soon as awareness detaches itself from the body, this disappears. Only the hair and the nails remain, everything else dissolves. In the case of some great masters, the body begins to shrink after death and disappears within a week, apart from the nails and hair. All these phenomena are associated with the realization of the rainbow body. (....) This is how they die the great adepts of the Dzogchen tradition, where the rainbow body is a well-known phenomenon.

Among the listing on the Stock Exchange of a hyper-technological Promised Land of Immortality and the reality of our being in the flesh, it seems that not all that glitters is gold, and the result is, as Timmermans suggests [3], questionable: (...) people in Western societies at the turn of the twenty-first century have turned away from personal, community-centered dying and embarked instead on an elusive search for the postponement of death. Instead of passing away among relatives and friends with whom we have shared long histories, we pay white-coated professionals to deal with the sudden-dying process. Their work is secluded behind closed doors, and their task is to keep death at bay (...) A society that builds and supports an extensive resuscitative system opts for aggressive intervention, death defiance, and medicalization of the dying process.

\section{Aphysical Frame of Reference for Consciousness and the Mind-Body System: The Building-Blocks Model}

When a rational soul is present in this machine it will have its principal seat in the brain, and reside there like the fountain-keeper. Descartes $(1633)^{13}$

The investigation and interpretation of reality may have several paradigmatic models of reference. The most studied and applied model in the fields of science, including neuroscience, namely Building-Blocks Model (BBM), is based on Descartes' dualism and classical Physics, which are also the pillars of Neuronal Correlates of Consciousness (NCC).

The BBMis a deterministic theory conceived in the womb of Positivism, which is based on the postulates of classical Physics (that have been proven wrong by General Theory of Relativity and Quantum Physics), which is based on the Cartesian dualism res extensa vs res cogitans (which leaves to God what belongs to Him, the res cogitans, and gives to classical Science what is of classical Science, the res extensa, forcing science to exclude a priori the mental phenomenon from its field of investigation), which is the post-Galilean expression of the work of rehabilitation of Platonic thought carried by medieval Thomism.

As a deterministic theory, the BBM is matching the doctrine expounded by the mathematician and astronomer Pierre-Simon de Laplace in Système du monde (1814). This doctrine, which would dominate the scene until the conceptual revolution caused by quantum physics, can be summarized as follows:

1) the universe is governed by causality,

2) in the history of the universe every state of matter is determined by what ${ }^{13}$ Descartes, Treatise on Man, 1633, $\$ 131$

https://www.colorado.edu/neh2015/sites/default/files/attached-files/descartes-treatise_on_man.pdf 
precedes and determines the following, in a manner analysable by means of Newtonian mechanics;

3) causality allows predictability;

4) the phenomena are described according to the mechanistic and deterministic paradigm which draws strength from the precision and rigor of Newtonian mechanics;

5) the case does not exist, it is a concept relative to the limits of human capabilities: it is inconceivable that there are events which fall outside a law,

6) the causal network of the Universe is however too complex for it to be reconstructed by the human mind; because of this all the physical knowledge can only be based on probabilities (approximations for lack of data).

The fundamental purpose of classical Newtonian physics was to causally explain the action of the forces on the particles that compose the cosmos, and to describe their reciprocal motion with the aid of mathematical equations. From the three laws of dynamics and from the law of universal gravitation we obtain the equations of motion, which allow us to predict, with reference to the three dimensions of space and the one-dimensional flow of time, the trajectories of the bodies. On this basis, deterministic links are established between the bodies, according to the principle of a necessary connection between cause and effect, which can be expressed through the equations of motion. Nothing happens by chance in the Newtonian Universe, but everything is necessary as subject to the deterministic laws of Nature.

Newton supposed the existence of an absolute, infinite, empty, three-dimensional, invisible space, not modifiable by the masses and firm with respect to their motion. It was thus possible to measure the uniform motion of a body with respect to this absolute space. Each body occupies a determined and unique position in this absolute space, and two bodies can occupy the same space, but only at different times. Time thus measures the succession and simultaneity of events in space. And like space, Newton's absolute time is conceived in mathematical terms: it is composed of a continuous series of instants, arranged in a single dimension. The time for Newton is a physical reality, universal and absolute, not alterable from the bodies and their motion.

The world seen through the Newtonian and later on positivist paradigm is a world made up of deterministic elements of reality that interact with other deterministic elements of reality. The structuring of the objects and the phenomena that we observe inside and outside of us, would originate from the different combination of fundamental and irreducible elements of reality (like the atoms of Democritus). Each combination is arranged by a code (algorithm), that is, by a sequence of operations that when applied to certain initial data lead to a certain final result. The structuring of objects and phenomena would take place according to the scheme, reductionist and mechanistic, used in the conception, design and construction of a mechanical system: a certain combination of building blocks generates a certain structure, which generates certain processes that, 
thanks to the input of energy (the capacity of a physical system to do work, i.e. the capacity to cause movement, change), make the ... machine work.

According to this model, there are fundamental, irreducible pieces of reality conceived as tiles of a mosaic (building blocks). From their combination, derive the objects and phenomena that we observe, inside and outside of us. Each different combination is headed by a code. If you know the code, and you have the material to combine (building blocks) and the tools to operate, you can artificially reproduce that particular combination of elements. Following this mapping out of reality, scientists of yesterday and today are hunting for building blocks and codes to assemble them, at all levels of matter, subatomic, atomic and supratomic, inorganic, organic, biological, cellular, of organ and system, of mind and consciousness (AI is based on this assumption).

They are seeking pieces and codes of all kinds (the so-called mirror neurons are on the list), including pieces of concepts, pieces of consciousness, pieces of thought, pieces of life and the codes for the architecture of brain and cells and the like. They assign a primary ontological value to the building blocks, and consider the relationships and interactions that we observe as a fact derived and subordinate to their existence. In this perspective, for modern molecular biology phylogeny is a strong succession, logic and computational, of biochemical events orbiting around the building blocks of the biological phenomenon, the nucleotide bases, the alphabet of the language of life (A, G, C, adenine, guanine, cytosine and T, thymine in DNA; A, G, C and U, uracil in RNA), from which combination and recombination would derive the phyletic descent (to this line of thinking belongs the belief, denied by evidence, that the complexity and the evolutionary degree of a species is directly proportional to the number of genes present in its genome).

An atomistic-like ontology that does not want to give way to evidence, as Hans Primas points out [37]:

(...) the naïve reductionism which tries to explain all phenomena in term of entities at a supposedly lowest level of theoretical description is still popular. This approach fails simply because the presumed lower level entities do not exist in a theory-independent sense. Modern quantum mechanics put an end to atomism. The so-called "fundamental' entities (such as electrons, quarks, or gluons) represent "patterns of reality", yet they are not building blocks of reality.

Anyway, one thing is to recognize that ill-defined, temporally variable, hard-to-quantify traits such as MBS and consciousness rely on the availability of specific phylogenetic requirements carried on and transmitted by the genic activity (which, despite the enormous progress made by techniques and technologies intended to manipulate $i^{14}$, remains far from being understood), another thing is to identify these traits with it, which is not just a misleading idea, but

${ }^{14}$ Today scientists are able to modify the germinal lines through a revolutionary genetic editing technique known as CRISPR-Cas9, a technique that, according to some scholars, e.g. Robert Pollack [38] can open on the return of the eugenic program: the positive selection of the "right" (fit) versions of the human genome and the elimination of the "wrong" (unfit), not only for the health of an individual, but for the future of the species. 
patently a wrong one.

Nigel Goldenfeld and Leo Kadanoff are suggesting to Use the right level of description to catch the phenomena of interest. Don't model bulldozers with quarks [39]. While it is certainly true that all the properties of a bulldozer result from the particles that make it up, like quarks and atoms, it is useless to think about the properties of a bulldozer (its shape, its color, its function) in terms of those building-blocks. Certainly no one would think of associating a ferrous meteorite fallen on our planet to the possible presence (past or present) of extragalactic bulldozer, stating that: Without iron atoms no known form of bulldozer could be realized! Yet there are plenty of experts who associate the recent discovery of the presence of salt water in the liquid state on Mars [40] with the possible past (or present) presence of (biological) life on the red planet, stating that: Without water, no known form of life is possible! To isolate the variable "water" from the complex of variables, still unknown, which have allowed the terrestrial biological phenomenon to develop, treating water in the liquid state as the elective environment-medium for life in the galaxy is not only very reductive, but quite ridiculous. A line of thought that does not cease to surprise, given that also recently some experts in the field [41] believe that the organic matter, i.e. pre-biotic organic building-blocks of life, found in meteorites (Carbonaceous chondrites) that formed during the birth of the Solar System 4.5 billion years ago may provide key clues to understanding the birth of life here on Earth (a belief in line with the theory of panspermia, according to which life on Earth originated from microorganisms or chemical precursors of life present in outer space and able to initiate life on reaching a suitable environment).

Well, this is a very interesting paradigm, but it is not what Physics suggests today. It is what classical Physics prescribed more than a century ago, before it was showed to be wrong.

\subsection{A Physical Frame of Reference for Consciousness and the Mind-Body System: The Quantum Model}

... quantum theory itself is intrinsically psychophysical as designed by its founders, and as used in actual scientific practice, it is ultimately a theory about the structure of our experience that is erected upon a radical mathematical generalization of the laws of classical physics. Henry Stapp, Mindful Universe: Quantum Mechanics and the Participating Observer (2011).

The scientific revolution triggered by the formulation of Einstein's Theory of General Relativity (which has radically changed what we know about space and time) and Quantum Mechanics (which has profoundly changed what we know about matter), reveals a microscopic and macroscopic physical reality completely different from that theorized by Newtonian physics, which loses the title of universal physical theory and must be content to maintain a practical validity only mechanically, i.e. on the mesoscopic plane of linear relations (predictable, reversible, reproducible) elapsing within a fixed and passive space containing 
localized material entities whose movement in time is deterministically governed by mathematical laws.

Albert Einstein showed that classical mechanics was based on two wrong assumptions: 1) that the measurement of time between events was independent of the observer's motion; 2) that the measurement of the space between two points of a reference system was independent of the observer's motion. It follows that the laws of Newton's dynamics must be profoundly reviewed: these in the strong sense can no longer be considered true descriptions of natural phenomena. The new theory that replaces the dynamics of Newton will be called theory of special relativity (SR). If SR is true, with its principle of the constancy of the speed of light, then this is incompatible with Newton's force of gravity, which acts instantly at infinite distances. Einstein worked on the problem for several years, in 1915 he was holding the solution: the theory of general relativity (GR). The principles of SR were extended to accelerated motions, whereas originally they referred only to uniform motions (which always represent idealizations). Since all masses are subject to gravity, and therefore are accelerated (they are never really in uniform motion, or inertial), relativistic laws are enforced for the entire universe. SR challenged the intuitive nature of the concept of time. Now the same fate had to do with the intuitive idea of three-dimensional Euclidean space. Newton's space was in fact replaced with a new geometry of space, a continuous four-dimensional space-time. The geometry of the cosmos is non-Euclidean. Despite being far from common sense and despite having questioned the fundamental concepts of Newton's mechanics, because of its determinism the Einsteinian theory of relativity is still a classical theory, and a realistic one, as it considers the laws of nature independent of the observer.

At the beginning of the twentieth century began a new research perspective, in addition to the relativistic one, which ended with the construction of the quantum theory of matter. This theory completely destroyed the building of classical physics, and replaced determinism with quasi-determinism, causal explanation with probabilistic explanation, and introduced the observer as a subject not merely passive in the laws of physics (a variable unrelated to classical physics, which seems to assign to the mental action exercised by the observer the ability to interfere with the object of his/her observation, i.e. the ability to affect the experimental result, a hasty and misleading conclusion contradicted by evidence: to interfere is the "measurement" action itselfregardless of who makes it, human observer or electronic detector as in the Weizmann experiment ${ }^{15}$ ).

Einstein's relativity, since its appearance, introduced very strong elements of discussion in the epistemological debate, concerning the role of the observer, the status of natural laws, the process of formation of scientific concepts and their value. The same can be said, a fortiori, for quantum mechanics (QM). For two cen-

${ }^{15}$ Nevertheless: The measurement problem arising from the quantum experiment does not necessarily imply that something "from the mind of the observer" affects the external physical world. The measurement problem does, however, hint that there is more to say about the physical world than quantum theory says [42]. 
turies classical mechanics had been a great success in explaining phenomena; it was considered a true theory, a marvelous building erected by human intelligence. Now this theory proved to be false, incapable of explaining ultra-macroscopic, galactic, and ultra-microscopic, electromagnetic, and sub-atomic phenomena. In its place there were theories that introduced the relativity of time and space, quantum indeterminacy, wave-particle duality, entanglement, bosonic superposition ${ }^{16}$ and other "strange" phenomena. A series of phenomena and conditions which, despite being unobservable by, and incompatible with, the deterministic vision of reality provided by classical physics, continue to be explained by deterministic laws that instead of being based on a logic of relations linearly determined, are based on a logic of relations probabilistically determined, leaving to the Heisenberg uncertainty principle the task of occupying the only (quasi) indeterministic box of the whole theoretical system.

In recent years, the twentieth century epistemological disorientation created by the paradigmatic change has been largely overshadowed by tremendous scientific and technological progress on the ability to derive detailed knowledge on the structure of a system, but the ability to integrate this knowledge has been hampered by the absence of a suitable conceptual framework, within which to describe the qualitative behavior. Even when you have a complete map of the nervous system of a simple organism, such as the nematode, it is not possible to derive its behavior. Likewise, if it is useless to reduce the properties of a technological artifact like a bulldozer to those of the atoms which compose it, it is even more senseless to reduce the complex behaviors and traits of an alive and vibrating organism to the properties of the pre-biotic organic building-blocks of life. Behaviors and traits of an organism are emergent properties of the biological system's endogenous and exogenous relations as a whole, which does not correspond to the sum of its parts, nor to the sum of the properties of each component taken apart from the whole.

The hope that physics can achieve completeness through an ever more detailed understanding of physical forces and fundamental constituents is groundless. The interaction of components at a given scale can result in a complex global behavior on a larger scale, which in general can not be derived from the knowledge of the single components or sequences of components. Basically, by replacing deterministic sequences with probabilistic sequences nothing really

\footnotetext{
${ }^{16}$ The particles described by symmetrical wave functions are known as bosons and obey the statistics of Bose-Einstein. The particles described by anti-symmetrical wave functions are known as fermions and obey the statistics of Fermi-Dirac. Quantum-relativistic mechanics demonstrates that the property of being described by symmetrical or anti-symmetrical wave functions depends on the nature of the particles. In particular it is unequivocally linked to their spin:

- particles with semi-wholespin are fermions (e.g. electrons, protons, neutrons); all the elementary particles that make up matter are fermions;

- particles with whole spin are bosons (e.g. photons).

All the elementary particles responsible for the forces that hold fermions together are bosons. In accordance with the Pauli exclusion principle, two fermions can not share the same quantum state, while bosons can. This translates into a strong resistance to fermion compression. This resistance creates the rigidity of ordinary atomic matter.
} 
changes, reality continues to be described as being composed of sequences, which is false. Sequences of units are part of the map, not of the territory.

The epistemological disorientation of the first half of the twentieth century has paid off during the second half, and not because the first, devastating, atomic bomb was dropped, but because some assonances emerged between the new and overwhelming vision of reality outlined by modern physics and some assumptions of the newborn psychology, such as the Freudian concept of libido and distinction between conscious and unconscious, followed by the Jungian one between individual and collective unconscious (leading to the Pauli-Jung concept of Unus Mundus), intertwined with the nascent Information Theory and with the growing interest in Artificial Intelligence, stimulating a lively debate on the possible overcoming of the res cogitansvsres extensa Cartesian dualism, by resorting to the supposed unifying (eventually computerizable) contributionof quantum physics ${ }^{17}$.

That is, several hypotheses have been formulated over the course of a few decades, to explain the function of the brain within the framework of Quantum Mechanics, judging legitimate to ask whether quantum theory, the most fundamental theory of matter currently available, can help us understand conscious$n e s s^{18}$. A great effort that most often rests on a great misunderstanding: the brain, the mind, the psyche and the consciousness are exchanged for each other and with each other as if they were synonyms, when they are not. A big confusion therefore, which at date can only benefit the CPS (Cyber Physical System) market.

\subsection{A Physical Frame of Reference for Consciousness and the Mind-Body System: The Tenso-Relational Model}

Basically, mind is an emergence which requires the existence of these three phenomena or cycles: with the body, with the world and with others. The phenomena of mind can exist only within the cycle, in the de-centralization that it involves. Francisco Varela

The degree of complexity of a phenomenon is not an inherent property of the phenomenon itself, but a measure of the degree of difficulty we encounter in investigating, understanding and explaining it. In the field of neuroscience, it is a widespread and unanimously shared opinion that the brain is one of the most

\footnotetext{
${ }^{17}$ The basic building blocks of quantum theory are, then, a set of intentional actions by agents, and for each such action an associated collection of possible "Yes" feedbacks, which are the possible responses that the agent can judge to be in conformity to the criteria associated with that intentional act (...) In quantum theory the main idealization is not that every object is made up of miniature planet-like objects. It is rather that there are agents that perform intentional acts each of which can result in a feedback that may or may not conform to a certain criterion associated with that act. One bit of information is introduced into the world in which that agent lives, according to whether the feedback conforms or does not conform to that criterion. Thus, knowing whether the counter clicked or not places the agent on one or the other of two alternative possible separate branches ot the course of world history [43].

${ }^{18}$ For an overview see e.g.: Atmanspacher, H. (2015) Quantum Approaches to Consciousness, Stanford Encyclopedia of Philosophy https://plato.stanford.edu/entries/qt-consciousness/
} 
complex systems we know. The system brain belongs to the energy/matter, namely res extensa, domain.

The definition of mind, as well as that of consciousness, it involves describing something that is subjectively experienced in the first person and explaining it in an objective form in the third person. Since neither one nor the other is detectable or ascribable to any of the categories of the so far known energy/matter domain, objectifying them becomes a very hard task (the epistemological debate that resolves around the application of the Quantum Model is attempting to overcome the obstacle by ascribing the mental fact to the quantum domain). Due to the anthropocentric vision that characterizes all the post-Neolithic internal representations of external reality, whose commitment is to have full control over Nature and its destiny, a mind without consciousness is like a boat without a helmsman, at the mercy of the waves of the sea, at the mercy of Chaos. This is the reason why, from this period on, when referring to mind, mind endowed with consciousness is implied.

In most of the scientific literature mind-consciousness (belonging to the res cogitans domain) is considered an epiphenomenon of the body (particularly the brain). Brain is considered the seat of mind, mind is the software and brain its hardware. The current NCC approach tries to explain mind as a byproduct of physico-chemical processes involving the billions of neurons in the human brain and its various components are the thought process, emotions, intelligence and awareness. This approach it suffers from certain flaws in that it only defines and quantifies mind-consciousness by way of certain neuropsychological parameters and discusses it in terms of its correlations with cerebral states, characterized by electrochemical flow in certain circuits or neural networks, which may extend into multiple brain areas. It fails to explain its physical identity and the experiential aspect of the subjective state of being oneself, or the "I/ $M e$ " experience which everyone experiences in day to day life. Basically, it tries to explain something belonging to the res cogitans domain, by replacing the blocks of the theoretical building erected not to deal with it, but leaving its foundations intact.

Alternatively, by projecting the neo-positivist secular religion movie on a cyberspace's mandala screen it appears that there is who have understood everything there is to understand in order to combine Quantum Physics, Junghian psychology, Vedic doctrine and so on and so forth, coming to the conclusion that mind-consciousnessand res extensa comes together and represents the ultimate and universal reality, namely Universal Consciousness. So get ready because intelligent machines equipped with consciousness (androids) are around the corner.

The model I am going to apply to advance an explanation of MBS and consciousness, namely Tenso-Relational Model [44], it stems from applying my previous Endo-Dynamo-Tensive Model [45] to the hypothesis independently developed by me [46] [47] [48] [49] and by Sá-Nogueira Saraiva [50] [51] on the phylogenetic scenario that led to the settling of a distinct and relatively auto- 
nomous neuro-psychological identity of the human being, i.e. to the settling of an $I / M e$ vs Other than I/Me, and from adopting an approach to the interpretation of mind phenomena which rests on a framework outlined by multidisciplinary contributions, such as Ilya Prigonine's Complexity Theory [52], Luigi Fantappiè's Unified Theory of the Physical and Biological World [53] [54], Humberto Maturana's Biology of Cognition [55] [56], Robert Rosen's Relational Complexity [57] [58] [59] (later being called Relational Science or Relational Biology), Chris King's model of Supercausality [60] [61], Francisco Varela's Neurophenomenology [62], Emilio del Giudice and Giuliano Preparata's Super-Coherent Oscillatory State of Biological Water (see [63]), and Carl Gustav Jung's Analytical Psychology [64].

The Tenso-Relational Model is based on a Relationship Theory in which the only relevant fact under the psycho-physical profile is the relationship between different events ${ }^{19}$ (Alfred North Whitehead) in space-time. According to this model, each element of reality, at any level of observation, is a micro system of relations, set in a macro system of relations. Nothing of what we observe exists in and by itself. Everything we can think of, observable and unobservable (i.e. virtual), instead of being made up of building blocks, or even quantized, in its ground state consists, as I'm going to clarify, of tenso-relationship systems.

As I discussed in [45], the establishment of a physical dimension based on a relational dynamicit arises as a consequence of a cosmogonic event, namely the supra-liminal self-perturbation (tensorial symmetry breaking) of a primary, irriducible and intrinsically dynamic state of tension (Implicated Endo-Dynamo-Tensive State $\left.^{20}\right)$, cosmologically assumed as the physical background from which emerge all the differences in potential, all the interactions or forces and all the physical and psychical relationships. A second supra-liminal perturbation (tensorial transition) leads to the constitution of the spacetime system of correlative interactions between gradients of tension that we know as relativistic dimension or dimension of an exited tension-gradients distribution or ET-GD (mass and energy free dimension). A thirdsupra-liminal perturbation leads to the constitution of $a$ non-exited energy-quanta gradients distribution (zero point quantum field), from whose perturbation (supra-liminal oscillatory motions and/or charge densities of energy-quanta and impulse) takes shape the quantum and supra-quantum dimension (the dimension of the exited energy quanta-gradients distribution or

\footnotetext{
${ }^{19}$ An event is intended as concrete primary element of the Universe, a knot of relations neither isolated nor isolatable from the whole in which it is comprised.

${ }^{20}$ The irreducible form of the relativistic dimension in the quiet or non-excitated regime (N-EIRD, Non-Excited Irreducible Relativistic Dimension) constitutes the irreducible physical plane devoid of matter, energy, space, time, and can be described as a state devoid of structure (continuous, isotropic, homogeneous, imperturbed) and super-symmetrical, of a tension qualifiable as Implicated Tension (IT) which bears within it a protodynamic, undifferentiated and totipotent principle (dynamis). This state is indicated as an implicated endodynamotensive state. Normally, by tension is meant the effect or the state produced by a difference in potential or by the application of a force but in the context of the N-EIRD the Tension/dynamis is understood not as the effect but as the presupposition (super-symmetry of implicated tension) of all the differences in potential, of all the interactions or forces and of all the physical (energetic and tensorial) relationships.
} 
EEQ-GD), where the relation (ship) is somehow explicated and may possibly be observed and directly experienced.

The phenomenological becoming is marked by the coexistence of manifested states (explicated), and unmanifested states (implicated) (David Bohm), and by the alternation of rarefied states and condensed states, whose dynamics respond to the prescriptive but not descriptive action of a poietic principle (which produces development and structure) called self-organization. The action exerted by self-organization is contextualized in the constitution of physical objects called attractors, syntropic nuclei (inducing coherence [65] [66] [67] [68] in the dynamic of a system $)^{21}$ that perform a catalyzing action on the dynamics of the confinement processes, giving them a preferential orientation, and is supported by the availability of an acting principle which, through recourse to polarized hysteresis ${ }^{22}$ domains, heading to standing mnesic-like processesof a chaotic type, introduces a variable and convergent quota of in-formation (negentropic action) ${ }^{23}$, namely mnemotropy. The combined action obtained via the 1) poietic

${ }^{21}$ By syntropy (a symmetrical law of entropy) is meant the degree of internal organization of a living system connected to a set of elements or factors that change in unison according to a certain orientation (not randomly but coherently, e.g. via Phase Conjugate Dynamics, Spin Conjugate Dynamics, Tension Conjugate Dynamics).

${ }^{22}$ Hysteresis is a non linear phenomenon that leads to the absorption and registration by a system of a part, at times infinitesimal, of the effects provoked by a given perturbative event applied to the system itself. The phenomenon of absorption and registration of the variations in state connected with the exposure to the perturbative event means that the response of the system to a new perturbative event depends also to a variable extent on the chronological history of all its variations in state and all its previous reactions to the current exposure. It can be deduced that the physical sizes relating to the perturbations applied are not perfectly reversible (they have a non-linear pattern) and describe curves known as hysteresis curves or cycles. In biological phase transitions (pointing at autopoiesis), transitions never occur linearly but always involve a degree of non-linear modification of the system itself so that the phenomenon of hysteresis becomes an integral part of the system's capacity for subsistence and not a limit. In the relativistic context the phenomenon of hysteresis assumes a central role in the genesis of both space-time and the energy phenomenon. In this context the progress of the hysteresis cycle met by the fractal/holographic objects that make up the territory of space-time are described by the becoming of the relationship between tension and torque, and the corresponding diagram is derived from the variations revealed by the Twisted-Pinched Hysteresis Loop [45].

${ }^{23}$ As I discussed in [18] thanks to the success obtained by C. Shannon's Information Theory in the field of Telecommunications and computer science, the improper use that the theory makes of the term "information" has been adopted, practically with a copy-paste operation, in all areas of science. Shannon's Information Theory did not answer to "What is information?", it only answered to "What amount of information, i.e. data, may be transmitted in time unit using a set of certain signals?". Shannon's Information Theory, together with Norbert Wiener's cybernetics ("Information is information, not matter or energy, it is something else", he wrote), namely thescience of control and communication in the animal and the machine, have legitimized the misguided use of "data transmission", or even worse of transmission of messages containing a (semantic) meaning, as a synonym of the physical concept of "information". It must be stressed out that the physical concept of "information" it has absolutely nothing to do with that of "data transmission", and even less with that of "transmission of messages containing a (semantic) meaning". The former consider "information" as a measure of coherence or structural "complexity" of surrounding system related to various entropic processes in physical world, that is the measure of in-formation amount, related to a certain object, may be a complexity of its internal structure (negentropy), while the latter consider "amount of information" as frequency characteristic of code letters-signals, that is improving of messages coding and decoding methods and solving of other questions related to optimization of technical communication systems operation. But the damage has done, so cells are communicating to each other, the genome contains the alphabet of life set up by letters, the brain sends messages to, and receives messages from, the whole body, etc. 
action (which produces development and structure) namely self-organization, 2) syntropic action (which induces coherence in the dynamic of a system) which translates into the formation of syntropic nuclei called attractors, and 3) mnemotropic action (which introduces a variable and convergent quota of space-time dependentcausal continuity coupled to a variable and convergent quota of unpredictable discontinuity), based on polarized hysteresis domains heading to standing mnesic-like processesof a chaotic type, is at the base of the overall state of every confinement process or system of confinement processes of phenomenological becoming. Each form of manifestation, at any level, is in a state, referred to as state of mnemopoiesis or mnemopoietic state, which is a function of the degree and level of integration and synergy elapsing between these three actions.

In the exited energy quanta-gradients distribution (EEQ-GD) regime every object or phenomenon is assimilable to a vibrational system (describable via a mathematical tool known as wave function $\psi$, an abstract mathematical function that contains all the statistical information that an observer can obtain from measurements of a given system ${ }^{24}$ ), that vibrates with a certain frequential configuration, a certain oscillatory or phase modality (rhythm of oscillation) and a certain intensity, maintaining an uninterrupted local and non-local relationship of interference ${ }^{25}$ with other vibrational systems.

The phenomena of interference between the oscillatory modalities of the energy flows and impulse involved in the perturbation/excitation of the quantum field give rise to coupling-phase (oscillatory resonance ${ }^{26}$ ) able to trigger the phase transitions ${ }^{27}$ (here "phase" is referred to the rotation angle and to the dy-

${ }^{24}$ Consequently, referring to the collapse of the wave function as if it were a process or phenomenon belonging to reality is nonsense: the wave function isan abstract mathematical function that contains all the statistical information that an observer can obtain from measurements of a given system.

${ }^{25}$ Wave interference is a phenomenon that occurs when two waves meet while traveling along the same medium. The interference of waves causes the medium to take on a shape that results from the net effect of the two individual waves upon the particles of the medium. Wave interference can be constructive or destructive in nature. Constructive interference occurs at any location along the medium where the two interfering waves have a displacement in the same direction. For example, if at a given instant in time and location along the medium, the crest of one wave meets the crest of a second wave, they will interfere in such a manner as to produce a "super-crest". Similarly, the interference of a trough and a trough interfere constructively to produce a "super-trough". Destructive interference occurs at any location along the medium where the two interfering waves have a displacement in the opposite direction. For example, the interference of a crest with a trough is an example of destructive interference. Destructive interference has the tendency to decrease the resulting amount of displacement of the medium.

${ }^{26}$ In physics resonance or coupling-phase is a condition under which an oscillating system responds to an alternative driving force with the maximum amplitude. Such condition may exist when the frequency of the driving force matches the natural (non-damped) oscillatory frequency of the system. Thus, in case of an imposed oscillating electromagnetic field, a biological system (e.g., a cell) will respond in a measurable manner only to those exogenous oscillations (i.e. alternative driving force) that match the natural (endogenous) EM oscillations of such system.

${ }^{27}$ There is a concept of phase states in physics. In general case phase relates to possible descriptions of physical system at changing of its parameters on which this system depends (e.g. temperature, type of space, time, string tie constant in string theory, etc.). In respect to a matter phase state means one of possible states of the matter: solid, liquid and gaseous. Changes from solid to liquid and from liquid to gaseous and viceversa are known as phase transitions. 
namical regime in which the system lives at a given stage of its evolution) that lead, according to QED (Quantum Electrodynamic Field Theory), to the structuring of matter (domains of oscillatory coherence vs domains of oscillatory incoherence). In particular, each localized (in space and/or in time) form of confinement (tensorial, energetic, massive, subatomic, atomic, supra-atomic, biological, cosmological), i.e. delimited by a boundary ${ }^{28}$, is a tenso-vibrational micro-environment and corresponds to an oscillator or a resonant cavity (cavity resonator), a stationary system organized around a particular tensorial/frequential configuration of perturbations (tensions/oscillations), existing thanks to the relationships of interference it has with the endogenous and exogenous tenso-vibrational environment ${ }^{29}$.

The ordinary dimension (Middle Dimension) to which we are part of, is then defined as the dimension of condensed matter (atomic and supra-atomic organization of fermionic quantum states) and can be treated as a massive fractal oscillating system affected by four orders of phenomena ${ }^{30}$ relatively autonomous and independent, associated with just as many physical varieties:

- Tensorial phenomena (tensorial varieties. differentiated vs undifferentiated tensions; qualia; images)

- Energy phenomena (electrodynamic varieties. wave fields and matter fields; wave-particle duality; anti-symmetrical/chiral composite quantum states and symmetrical/achiral composite quantum states)

- Condensed matter phenomena (thermodynamic varieties, gas/liquid/solid, and chemical varieties, inorganic/organic)

- Biological phenomena (autopoietic varieties, prokaryotic and eukaryotic organisms)

and by three types of correlative dynamics or couplings.

1) a coupling of a frequency (fermions) $\leftrightarrow$ phase (bosons) type, between the massive plane and the wave-particle plane or kinetic gradients distribution (Phase Conjugate Dynamics of the type Frequency-Phase Correlative Dynamics);

2) a coupling of the phase (bosons) $\leftrightarrow$ tension (dipolar tension gradients) type, between the wave-particle plane and the dipolar tension gradients distribution (Spin Conjugate Dynamics of the type Phase-Tension Correlative Dynamics);

3) a coupling of the tension (dipolar tension gradients) $\leftrightarrow$ tension (monopolar tension gradients) type, between the dipolar tension gradients distribution

\footnotetext{
${ }^{28}$ Every structuring process of a system of correlations endowed with a degree of subsistence (condition of resonance) such as to make it distinct and/or distinguishable (even when not observable) from the context of the relationships it forms part of; in general, a confinement process is equivalent to a phenomenon of localization.

${ }^{29}$ In this sense the terrestrial environment is to all effects a tenso-vibrational environment and every biological structure/system corresponds to an oscillator/resonant cavity tuned on the particular tenso-vibrational configuration of the environment to which it belongs.

${ }^{30} \mathrm{Of}$ which one, mass and energy free, hypothesized and three known.
} 
and the monopolar tension gradients distribution (Tension Conjugate Dynamics of the type Tension-Tension Correlative Dynamics).

The interaction between Phase Conjugate Dynamics and Tension Conjugate Dynamics takes place only (with some exceptions) via Spin Conjugate Dynamics, which means that the interaction between the massive plane "body" and the tensorial plane "mind", takes place thanks to the interface established by quantum dynamics (Quantum Brain), which are in no way assimilable to "mind" dynamics (the so-called Quantum Mind is one of the inebriating side-effects induced by the seductive charm of quantum revolution).

\subsection{At the Roots of Mind: A Phylogenetic Frame of Reference}

Phylogenetically, as I discussed in [18] [44], through Nervous Tissue (NT) and Nervous System (NS) the organism may develop the ability to interface biunivocally the territory of tension with the territory of energy, so as to give way to new relational and adaptive strategies (new compared to the non or pre-neurological relational and adaptive strategies). In this sense, NT-NS as a unit is a selective interface between the two territories, energy $\leftrightarrow$ tension, and the original function assigned by cell differentiation to nerve cells is receptorial and meta-physical (of interfacing between one plan and the other of reality): that is, NT and the NS, regardless of the number of anatomical/functional units (neurons, glial cells and the like) involved, are condensed (fermionic) systems specialized in interfacing the Exited Tension-Gradients Distribution (ET-GD) with the Exited Energy Quanta-Gradients Distribution (EEQ-GD), and viceversa, for adaptive purposes. Photons (massless objects) and phonons (quantized mode of vibration) exchanges generated by the neuro-electro-chemical activity, are at the basis of this interfacing dynamic.

With respect to the territory of energy, NT-NS makes a selection (receptor function) species-specific upon the state variations or stimuli (perturbative events, e.g. frequency variations, phase variations, tension variations) from the external and internal environment, making the organism appropriately sensitive to a certain range of state variations and not to others. Moreover, NT-NS is specialized in transforming, upon kinematic similarity, the chemical and physical state variations of the environment, in spatial and temporal neuro-electro-magnetic phase correlations. With respect to the territory of tension NT-NS it transmutes the stimuli from frequencial pattern (receptor activity $\leftrightarrow$ neuro-electro-chemical activity) in isomorphic pattern of spin, on which operates a specific Holographic Strange Attractor $^{31}$ [69] [70] [71], which transmutes the pattern of spin in tensorial pattern, and viceversa, and sets the functional correlations between them, valid under the biological and/or neurological and/or psychic standpoint.

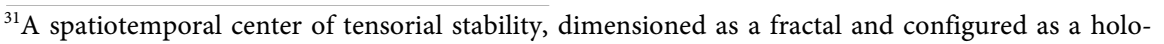
gram, around which it occurs the indeterministic variability of the coupling relationships (frequency-phasecoupling on quantum level; phase-tension coupling on quantum-relativistic level; tension-tension coupling on relativistic level).
} 
In general, a Nervous System defines and separates this network of interactions, which are of the chaotic-type (deterministic chaos) [72] [73] [74], from the environment, so that it can realize an autonomous but not isolated unit, namely a living neurologic organism. Humans, and to some extent also other vertebrates, are engaged in engramming a sensori-neural warp of tensions on which it can weave the weft of a variably integrated state ofmind.

In vertebrates, thesensori-neural responsecommon to all neurological organisms is processed and integrated, e.g. via super-complex anticipatory systems ${ }^{32}$, to become perception (perception being a function more integrated than sensitivity which is a function more integrated than reacting which is a function more integrated than excitability), which in humans is associated to sensing, the psychic-mental substrate on which thinking is grafted.

So when I say that the mental phenomenon belongs to the tensorial phenomena class, does not mean to say that the latter presents the characteristics we attribute to the human mental phenomenon. Instead, it means that physically the mental phenomenon is given by the localization (a process of confinement established by the action of a specific neurological basin of attraction ${ }^{33}$ of the chaotic type-e.g. a riddled basin of attraction ${ }^{34}$-, acting on tensorial level) of a particular ongoing distribution of dipolar tension gradients, coupled (via correlative dynamics) to the localization (established by the action of a specific neurological basin of attraction, acting on wave-particle level) of the energy gradients distribution (see also Section 3).

A distribution of energy gradients which is tuned on the neuro-electrochemical activity, that transmutes the environmental physical variations (internal and external stimuli), filtered by the six sensory organs (the CNS being the sixth and the most important sense organ, specialized in interacting with tension state variations), into a non-linear neuro-compatible stream of energy and tension gradients.

So when we refer to mind, we are not referring to something made up of something, or to the product of a mind-body mechanic, but to a phenomenon

\footnotetext{
${ }^{32}$ As Rosen writes: An anticipatory system is a system containing a predictive model of itself andlor its environment, which allows it to change state at an instant in accord with the model s predictions pertaining to a later instant [75].

${ }^{33}$ Roughly speaking, an attractor of a dynamical system is a subset of the state space to which orbits originating from typical initial conditions tend as time increases. It is very common for dynamical systems to have more than one attractor. For each such attractor, its basin of attraction is the set of initial conditions leading to long-time behavior that approaches that attractor. Thus the qualitative behavior of the long-time motion of a given system can be fundamentally different depending on which basin of attraction the initial condition lies in (e.g., attractors can correspond to periodic, quasiperiodic or chaotic behaviors of different types). Regarding a basin of attraction as a region in the state space, it has been found that the basic topological structure of such regions can vary greatly from system to system. [Credit: Ott, S. (2006), Scholarpedia http://www.scholarpedia.org/article/Basin_of_attraction]

${ }^{34} \mathrm{~A}$ type of basin topology that may occur in systems that, through a symmetry or some other constraint, have a smooth invariant manifold, i.e. there exists a smooth surface or hypersurface in the phase space, such that any initial condition in the surface generates an orbit that remains in the surface [76] [77].
} 
literally implicated in the human neuro-psycho-physiological relationship elapsing between its outer-outsight (embedded) and inner-insight (embodied). Accordingly, when we refer to consciousness we refer to the warp (namely the possibilities, skills and attitudes) on which is interwoven the weft of the ongoing faculty and ability to ideate, program and engage, not occasionally and not by domestication nor by artificial programming, an adaptive and supra-adaptive behavior not ruled by the phylogenetic prescription (behavioral stereotypy).

This presupposes that for there to be an adaptive and supra-adaptive behavior not ruled by the phylogenetic prescription, there must be a working "I/Me" which perceives itself as something else from an "Other than I/Me", and polarizes the Ego accordingly. This presupposition of consciousness is precisely the self, the psychic center of awareness. consciousness and awareness are not synonyms at all! Phylogenetically and ontogenetically awareness precedes consciousness, this also means that all animals are endowed by a species-specific degree of awareness tied to phylogenetic prescription, that allows them, without being endowed by consciousness, to survive across the ontogenetic and phyletic space and time. The only animal endowed with phylogenetic presuppositions that allow it to free itself from the phylogenetic diktat and to undertake a (very difficult) path of epigenetic modulation of the self is the human being. On this irreversible deviation (bifurcation) from the phylogenetic bond, Homo Sapiens has been able to develop what we call consciousness. An acquisition that is not devoid of deleterious effects, since Homo Sapiens et Faber has arrived to this days doing a great deal of damage, not to mention the damage he is about to do by hanging himself on the chips of artificial devices endowed with an algorithmic consciousness supposed to simulate his own.

\subsection{The Human's Mind-Body System}

I view the brain not as a box with compartments that contain sadness, joy, colour, texture, and all the other "objects" and categories that one might think of. Instead, I envisage it as a constantly shifting dynamic system; more like the flow of a river in which patterns emerge and disappear, than a static landscape. This is an entirely different image from the brain as a computer with stored contents or subroutines to be called up by a program. In nature's pattern-forming systems, contents aren't contained anywhere but are revealed only by the dynamics. Form and content are thus inextricably connected and can't ever be separated.

\section{J.A. Scott Kelso}

By mind-body correlative dynamics is understood a non-locally recombining weft of tension and energy gradients, filtered by the mind-body mnemopoietic state of the subject experiencing it, to become a virtual (intrinsically non-observable) stream of images and vortices, which we call sensations, perceptions, thoughts (see also Section 3). When we sense, perceive, think, we literally "become" those sensations, perceptions, thoughts. There is not something or someone separated 
from the mind-bodycorrelative dynamics, which observes it from the inside or from outside. This dynamics is not codified somewhere, not between the DNA helixes, nor between the folds of this or that body district, of this or that neuronal area. It is not codified at all. Instead, it is implicated in, and belongs to, the whole mnemopoietic system that supports it.

To better visualize the multidimensional scenario within which mnemopoiesis it acts, we can resort to Augusto Sabbadini's metaphor of the pond and the fish ${ }^{35}$.

Imagine a muddy pond where we are fishing. A fish swims in the pond, but we can not see it because the water is cloudy. At a certain point the fish bites. We raise the barrel and see it attached to the hook. In such a situation we naturally suppose that, just a moment before biting, he came to be precisely at the point where the hook was. Until a moment ago we were not able to say where it was: its position was for us, in a certain sense, indeterminate. But it was not an intrinsic, irreducible indeterminacy. It was linked only to incomplete information on our part of a reality that was in itself determined.

Now imagine that the fish is a quantum particle and the rod, line and hook are a device that measures its position. Also in this case, until we perform the measurement, the position of the fish is indeterminate. But this is a different and more radical indeterminacy. Rather than a normal fish, the particle resembles a soluble fish, which, before biting, is found widespread throughout the pond, more densely in some places, less densely packed in others. The uncertainty of his position is not just a lack of information on our part. Where it is thicker we are more likely to catch it, where it is less thickened we have less chance. But his position is intrinsically indeterminate. Nonetheless, miraculously, when the soluble fish is caught, its widespread nature instantly condenses and precipitates into a real fish, perfectly localized, hanging on the hook.

Normal fish and soluble fish belong to the territory of energy. Normal fish belong to our middle dimension, the three-dimensionality pond, where time flows from a before to an after and where the concept of distance and of alternation still make sense, the one we experience through our ordinary senses, with respect to which the laws of classical physics apply. Soluble fish belong to the quantum dimension, the one we can not experience through our ordinary senses, with respect to which the laws of quantum physics apply, the pond where the concept of distance and of alternation, of space and time, completely lose meaning, dissolving into something which contemporary physics calls zero point quantum field. Normal fish (massive plane) and soluble fish (wave-particle plane or kinetic gradients distribution) interact via frequency (fermions) $\leftrightarrow$ phase (bosons) coupling (Phase Conjugate Dynamics of the type Frequency-Phase Correlative Dynamics).

Spacetime continuum is the pond of tension gradient distribution from which

${ }^{35}$ From his introduction to the Italian version of David Bohm's Wholeness and the Implicate Order [78]. 
both soluble fish and normal fish appear and disappear. In the pond of tension, i.e. the exited regime (ERRD, Excited Reducible Relativistic Dimension) of the ground state of phenomenological reality, the individual systems (normal fish) of the middle dimension, and the particles or groups of particles (soluble fish) of the quantum dimension, do not exist at all. There are neither separate nor quantized entities that interact with each other, but rather images that are constantly formed and dissolved as in a kaleidoscope, vortices that are constantly formed and dissolved as in the current of a river. Vortices and images exist only temporarily and are only a reality to some extent fictitious (virtual) and arbitrary, cut out, remixed and stretched in the turbulent tension gradients floating.

The mind-body system (MBS), namely the neuro-psycho-physiological dynamics caracterizing an alive and vibrating human being, it can be seen then as an autopoietic system strung between the Exited Tension-Gradients Distribution (ET-GD) and the Exited Energy Quanta-Gradients Distribution (EEQ-GD), governed by the syntropic action of an Holographic Strange Attractor, able to develop different degrees of integration and to carry out different functions, that responds to the laws of psychodynamics of non-equilibrium, that constantly redefines, sustains and reproduces itself. The autopoietic dynamic of the MBS is organized around biophysical autocatalytic patterns (i.e. self-accelerated) regulated by continuous and non-linear fluctuations of selective tension transfer between the outer-outsight (embedded) and inner-insight (embodied) environment. The trans-neurological selectivity is the central element of the autopoietic neuro-psycho-physiological dynamics. There is a diffuse and integrate neurological intercellular relationship system, supported by a dense network of physical and chemical signals (via synapses, gap junctions, intra-extracellular fluid, plasma membranes, Cerebrospinal Fluid) capable of interacting with the environmental substrate so as to produce the net of soliton waves, i.e. neuro-electro-chemical impulses or nerve impulses or action potentials, that qualifies the sensory/receptor system activity. Selective tension transfer between intra and extra environment is associated with the production and transduction of tensorial signals (coherent scans of tension-state variations or tenso-stimuli) that come into play in the tensorial interconnection and in the biophysical processes of the Nervous System tuning (via Phase Conjugate Dynamics, Spin Conjugate Dynamics, Tension Conjugate Dynamics): that is, all neuro-psycho-physiological in-formation dynamics is carried, transformed and made available as holographic spin-phase interference patterns on the surfaces of higher order hyperspace fields (e.g. on a hypersphere, a structure embedded in a $4 \mathrm{D}$ space, equipped with a double donut-like shape, which is invisible in the usual 3D dimensions) [79], which are non-locally resonant with the intermediate EM field generated by the neuro-psycho-physiological activity. Since this dynamics lies under the syntropic action of a Holographic Strange Attractor, all the mnesic traces (retention of the absent) that in-form its becoming will give shape to a subset of tension gradients, a fractalized holographic hysteroid, the psychic basin of attraction or anthropo-psychic basin of attraction of 
the human spieces.

If we assume that the phenomena generated by the transition between pattern of spinandpattern of tensionrefer toa inertial system generated by the actual transition, the so generated inertial system may be treatedas the event-horizon of the neuro-psycho-physiological information dynamics, where by event-horizon ${ }^{36}$ it is meant a de-localized virtual entity ${ }^{37}[80]$, the space-time boundary between different planes of manifestation (heremind vs body), which traces the limits of observability and comparability of the phenomena that lie before and beyond the event-horizon (tension vs energy).

If this is the case, the surfaces of higher order hyperspace fields lay on and in-form the human's neuro-psycho-physiological event-horizon.

\section{Out of Body Experiences and Electromagnetic After-Effects: General Premises}

What the final method of observation must see in the production of "background physics" through the unconscious of modern man is a directing of objective toward a future description of nature that uniformly comprises physis and psyche, a form of description that at the moment we are experiencing only in a prescientific phase. To achieve such a uniform description of nature, it appears to be essential to have recourse to the archetypal background of the scientific terms and concepts. Wolfgang Pauli, Letter to C.G. Jung (16 June 1948)

Basic organic compounds, such as biological water [63], proteins and nucleic acids, are semiconductors and possess the ability to activate charges without participation of ions [81]. Semiconductors are good converters of chemical, thermal and electromagnetic energy into electric energy, and the other way round. A semiconductor can be a quantum generator of electrons and photons. In an alternating electric field, proteins, amino acids, DNA, RNA, as well as plant and animal tissues are subjected to electrostriction, i.e. the mechanical deformation of a dielectric (insulator) in the presence of an electric field, and become quantum generators of phonons or acoustic waves (a phonon being a quantized mode of vibration). This occurs because the structures in question are at the same time piezoelectric (the ability of certain materials to generate an electric charge in response to applied mechanical stress) and pyroelectric (the ability of certain materials to generate an electrical potential in response to applied thermal stress),

\footnotetext{
${ }^{36} \mathrm{By}$ event-horizon Iam not referringto the cosmological one but to aglobal property of a space-timeinertial reference system in-formed by de-localized (in space and time) correlative phenomenon. According to the standard cosmological vision, an event-horizon is instead a phenomenon of space-time singularity (a single space-time point, a place-event where energy-matter, density and temperature are infinite), envisaged following Einstein's Theory of General Relativity, when a non-banal and singular gravitational phenomenon or a gyroscopic motion at relativistic velocity deform the space-time continuum determining an event-horizon.

${ }^{37}$ The event-horizon is a (continuous) null surface in space-time. The event-horizon is defined non-locally in time: it is a global property of the entire spacetime and must be found in a separate post-processing phase after all (or at least the nonstationary part) of spacetime has been numerically computed.
} 
which means they possess the capacity for thermal and mechanical polarization. Piezoelectrics convert mechanical energy into electric energy, while pyroelectrics convert thermal energy into electric energy. Acoustic effects may take place not only in piezoelectrics, but also in semiconductors. A semiconductor can manage electrons in their induced state. In such cases, non-radiative recombination transmits the energy to the molecular network in the form of quantum quantized mode of vibration. The transition from the induced state to the basic state has got thus two possibilities: the generation of photon or the generation of phonon.

At micro- and mesoscopic level life is a result of all the chemical, electrical, magnetic, optical and acoustic events occurring in the living organism, in the system of organic-like semiconductors, piezo- and pyroelectrics. Therefore life takes place not in a chemical or electronic system, but to some extent among these two processes, where photon exchange plays a central role.

Life processes and light are inseparable and internally connected due to their electromagnetic nature. Light plays a significant energetic and regulatory role in living organisms and in the entire ecosystem, for instance in photosynthesis, in the process of seeing, in biological rhythms, etc. Changes in the intensity of photon emission are functionally connected with disturbances of homeostasis and their measurements specify the state of organism's vitality and the capacity for environmental adaptation. The electromagnetic interface and photon exchange is at the basis of all biological processes and it is thanks to it that a biological system, from the less to the most complex, interacts with the environment [82].

In this sense, the electromagnetic interface is, at the micro-, meso- and macroscopic level, the fundamental sensory module of any biological system [83] [84] [85] [86] [87], namely EM sensory module (a biological ability that several marine and few terrestrial animal species favored by developing electroreception, used in electrolocation (detecting objects) and for electric signaling).

Nerve cell of living organism is a generator of electromagnetic radiation in ultrahigh range of frequencies with the wave length comparable with linear dimensions of the cell itself. We can consider nerve cell as rhythmogenic center with exogenic modulated frequency. Weak magnetic fields of CNS neurons play substantial role in the Spin Conjugate Dynamics creating the second signaling system in addition to the synaptic one. H. Puthoff and R. Targ [88] had established influence of weak magnetic fields on the CNS leaving aside sense bodies. In their experiments they proved that upon transmission of stimuli under absolute absence of sensory contact the role of intermediary was played by electromagnetic oscillation of very low frequency. Interaction of radiations of different cells under absence of an external signal takes place at the expense of resonance events (interference) between waves of different length provided that both ultrahigh harmonic (sinusoidal) and low frequency nonlinear (stochastic) oscillations 
are available. Their synchronization is provided by nervous system, and their coherence by tissue specialization of cell structures. Upon weakening control of CNS, for example, as a result of a mental disease or impact of neurotropic toxins these processes can be put in order by external stimuli, namely: visual, acoustic and chemical ones or electromagnetic radiation. Interaction of radiations is executed at molecular level as a result of alternation of relative position of molecules creating the cell in space (conformational transfers). At that an effect of resonance intensification of cumulative signal is achieved.

Aneurological organism, then, interface with the environment not only viareceptor system, but also via the whole organism conversion to electromagnetic energy (transitory or permanent physical consequence of a NDS or a NDLS may be electro-sensitivity, which may be caused by a physical alteration of the whole system supporting the EM sensory module, first of all of biological water's composition and dynamics).

Matter can present itself in nature in several different states or phases, e.g. gas, liquid and solid at meso- and macroscopic level, which are responding to thermodynamic laws, and plasmas, lattices, Bose-Einstein Condensate (BEC) for a bosonic gas [89], Condensate of Bardeen Cooper Schrieffer (BCS) for a fermionic gas, Fröhlich condensates for proteins and nucleic acids [90] [91], solitonic waves (solitons) [92], quantum spin liquids (an entangled soup caused by quantum fluctuations, made of quasi-particles known as Majorana fermions, in a high correlated spin liquid state, namely a soup of spin-wave packets reached via spin-orbit and magnetic momentum coupling) [93] [94] at microscopic level, just to mention a few, which responds to electrodynamic laws.

Each state or phase of matter can be qualified according to its internal structure or order, that is to say according to its correlative structure (e.g. gas is less correlated than liquid which in turn is less correlated than solid state), and the connection between one phase and the other may take place through phase transitions (phase transitions from one state to another changes the correlative state of a system but may not change its nature, as is the case of water, which is always water in both the liquid, solid and gaseous state). Phase is the parameter that qualifies the dynamics of a phenomenon in relation to its temporal course and in relation to its oscillatory properties, where at each phase or state corresponds a different energy level, a different vibrational configuration and a different interferential register.

When the trajectory described by the becoming of a dynamical system is going to be deflected by a phase transition, for a certain period of time the system is to be in a state at the phase boundary, a state that the eastern tradition calls bardo (which on the occasion of a NDS and a NDLS may look like a pleasant or unpleasant oneiric-like state), a bifurcation point beyond which the system changes, partially or totally, in a reversible or irreversible way, its formal and/or vibrational and/or tensorial identity.

Under this scheme, any elementary constituent of a system it can act one way 
or another depending on the correlative system it comes to be part of. Accordingly, are the correlative properties of the system that determine its behavior, and not the properties of its elementary constituent taken individually and summed up [95].

The biological and neurological phenomenon can be considered as anon-linear dynamical systems of a chaotic type, where the dynamics of non-equilibrium guarantee the transformation and the existence of the system itself. Chaos (deterministic chaos) is inherent to health, not to disease: the non-linearity of physiological flows (air, blood, nerve impulses, etc.) corresponds to the fractal (fractional) nature of the geometries of its anatomical supports (lungs, blood vessels, neural networks, etc.) and guarantees its self-regulation. Conversely, the disease is a form of order, which finds its definitive expression in death. Chaos does not threaten biological systems from the outside, it is a property of the systems themselves: it is at the base of their being autopoietic systems. The perturbation lies in linear equilibrium: the pathogenic agent introduces into the chaotic in-formation of the system an "ordered in-formation", a conditioned behavior ${ }^{38}$.

Life is the trajectory described by the non-linear, rhythmic and continuous transformation of biophysical and biochemical processes supported by the mnemopoietic state of the biological system and governed by the action exerted bya multiplicity of integrated basins of attraction (riddled basins) of the chaotic type and toroidal topology, ruled by as many strange attractors, sized as fractals and configured as holograms ${ }^{39}$, consisting of adouble layered energy envelope, i.e. an outer layer composed of a fractal plot of Frequency-Phase Correlative Dynamics interference ridges and an inner one composed of a fractal plot ofPhase-Tension Correlative Dynamics interference ridges, non-locally coupled (entangled) with the projection, on the biological species-specific event-horizon, of a fractal distribution of Tension-Tension Correlative Dynamics (tensorial core) (Figure 2).

As for human mind, a basin of attraction could be the metaphor that indicates a single flow of mental images (perceptions, sensations, emotions, thoughts, memories) immersed in the flow of the relationship of continuity that remains implicated in the apparent discontinuity elapsing between the experimenter and ${ }^{38}$ Death it can be considered as the loss of the closed loop between the biosystem's physical energy dimension and tension dimension, between its three-dimensionality and hyper-dimensionality. While experiencing an NDS, the biosystem it may be stimulated or shocked back into coherence loop with its tension dimension, and be restored to living functioning, as is the case, e.g., of resuscitated bacteria [96].

${ }^{39}$ The peculiarity of the dynamics of a strange attractor comes from its being governed by its fractai dimension, its temporal and/or space structure is not characterized by a succession of discrete units (from 1 to 2 , from 2 to 3 , etc.), and is not composed of contiguous units $(1,2,3$, etc.), but consists of measurable (deterministic) fractions (fractal = broken) of space and time, spaced without interruptions (in continuum) by non-measurable (chaotic) fractions of inter-space and inter-time. Its holographic configuration stems from the fact that each of its action plans can be considered a portion of an indivisible whole where, as in a game of mirrors, the part projects itself by its self-similarity in the whole and the whole in the part, with different degrees of resolution and angles of perspective. 


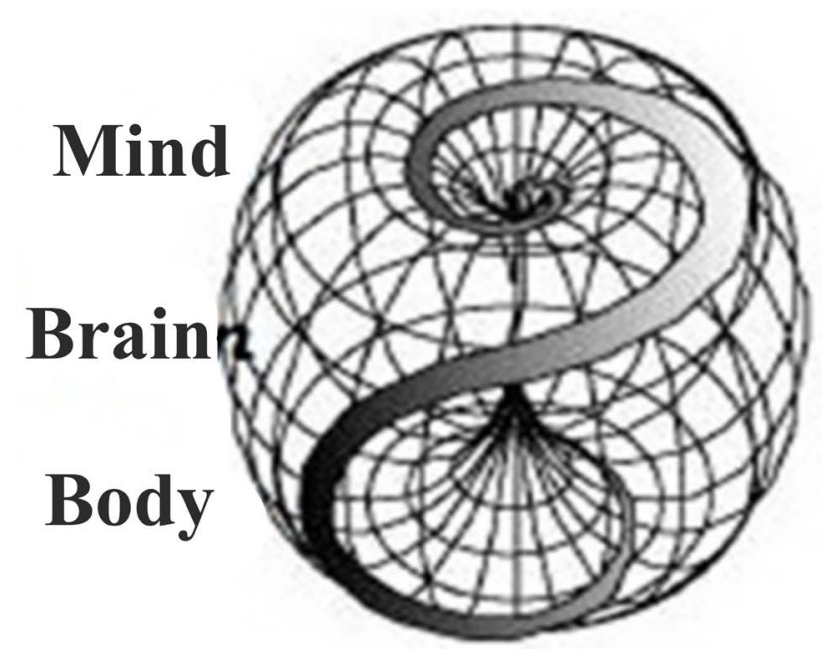

Figure 2. The human energy-tension basin of attraction, i.e. the mind-brain-body (de-localized) system, reproduces the fundamental structure characterizing the EEQ-GD, which in turn reproduces the fundamental structure of the ET-GD [45]. The fundamental structure of the EEQ-GD is a unitary fractal and resonant object, a toroidal-like cavity resonator, consisting of an outer frequency weft (Phase Conjugate Dynamics $\rightarrow$ Body), aninner spin weft (Spin Conjugate Dynamics $\rightarrow$ Brain) and a tensorial nucleus (Mind $\rightarrow$ Tension Conjugate Dynamics). On the ET-GD level, the precursor of this fractal object, is a diffusetoroidal vortex consisting of spin-internal motion (zitterbewegung, self-dynamism not dependent upon external factors), a stationary and fractional (non-quantized) entity within which the motion impulse is only latent. Subjected to a torque ${ }^{40}$ and rotational action (induced by reflection symmetry breaking) this diffuse toroidal vortex transform into a bi-polar quantum-relativistic object, named Twisted-Pinched Hysteresis Loop (T-PHL), in which the spin-internal motion is de-symmetrized in spin-up and spin-down. The final outcome (EEQ-GD) is a continuous and non-uniform cyclotronic motion of dextrorotatory vs levorotatory spin-angular momentum, that in passing through the de-localized point of self-intersection of the loop (the eight-like strip highlighted in the picture) decussate and reverses sign, moving seamlessly from negative values to positive values. The mirror symmetry breaking leading to the biological T-PHL is at the basis of the enantioselective absorption mediated by biological water, which determines biological homochirality.

the object of his experimentation, between the territory of energy and the territory of tension, between the body dimension and the mind dimension, and the continuous change of mental images could be compared to the passage of a relational trajectory from one basin of attraction to another. That is, this flow could be compared to a dynamic, fluid, non-linear chaotic type system that self-organizes around the prescriptive but not descriptive action of a basin of attraction, whose contextualization in subjective time makes it appear as fragmented into a multiplicity of integrated basins of attraction, where: even the smallest variation in flow performance or the smallest perturbation that acts on it, mediated by the selective and discriminative action of the neurological filter, is able to interfere

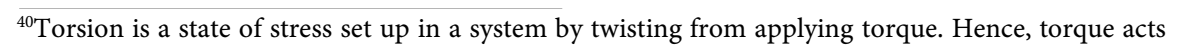
as a force and torsion as a geometric deformation. In our case tension super-symmetry acts as a twisting-force and torque as the fundamental stress of space-time geometry. 
in a not negligible way with the overall behaviour of the system.

In the phase space of a non-linear dynamical system of the chaotic type, the orbits ofits basin of attraction undergo a continuous stretching and bending process that mixes the orbits in an unpredictable way, giving the system an intrinsic character of uncertainty. The stretching and bending operation of a chaotic attractor systematically transforms the initial in-formation and integrates it with the following in-formation: the stretch amplifies the uncertainty on a small scale, the bending approaches trajectories (orbits) very far from each other and hides the in-formation on a large scale. The result of this continuous amplification and overlapping of the various oscillations that constitute the overall motion of the system, is precisely the unpredictability of its future behavior, the modus operandi (the problematization of solutions) of what in relation to phylogeny we call diversification, of what in relation to the neurological behavioral module we call overcoming of stereotypy and of what in relation to human behavior we call learning and creativity.

By their nature, all physical systems, and to an even greater extent biological systems, exercise their dynamism within limits of defined time and space, otherwise they would be eternal in time, and infinite in space. Establishing the limits of variability of time and space that delimit the existence of the system, these limits represent the limited variability of the system. Part of this variability, especially that which draws the ends (phase limits), does not manifest in the ordinary dynamism of the system. This portion of variability is called variability of reserves, and it coincides with the ability of a dynamical system to adapt and/or react to stress limits' evolution and perturbation (as during a NDS and a NDLS). That is, total variability (limited variability and variability of reserves), demonstrates the limit of the system's ability to behave not randomly (deterministic chaos), and sets the stress limits, specific to the system, beyond which its dynamic is not governed but random.

In an oscillating system in continuum (continuous rhythmic system), as a biological system:

1) the variability of reserve of the system identifies itself with the stress limits of each rhythmic property of the oscillating system,

2) limited variability coincides with its oscillatory ability,

3) oscillatory ability corresponds to the modulation ability of the rhythmic property of the system.

The modulation ability of the rhythmic property of the system includes:

1) tonic modulation or modulation of the average oscillatory level,

2) amplitude modulation,

3) phase modulation,

4) frequency modulation.

It follows that when the stress limits of a continuous rhythmic system are exceeded, i.e. when we have an over-modulation of one or more of the oscillatory properties, the continuous rhythm of the oscillating system loses its non-randomly 
cyclic behavior, to enter an irregular, messy, random dynamism.

In normal conditions, the cyclical processes activated by the species-specific basin of attraction are kept on a level of self-similarity, and scale invariance, a necessary condition for the functioning and morphology of processes and structures of a biological system, in order to remain functional in their assigned role, even though they transform (e.g., in the process of ageing structures and processes change, but always remain similar to themselves). For this purpose the riddled basin of attraction undergoes constant transformation from strange attractor (deterministic chaos) to limit cycle attractor ${ }^{41}$ (almost periodic) to fixed point attractor (periodic), and viceversa ${ }^{42}$.

As long as the degree of uncertainty of the species-specific basin of attraction and its syntropic function (ability to modulate the rhythmic properties of a system) are preserved (deterministic chaos), its self-transformation is reversible (e.g. physiological processes, processes of self-repair and healing), but when the degree of uncertainty and/or effectiveness of the syntropic function are compromised by internal or external causes (alteration or loss of ability to maintain the rhythmic properties of the system), the process of self-transformation can cease at the level of the limit cycle attractoror at the fixed point attractor's limit, with a relapse on the processes and/or structures that are under its action (as during a NDS and a NDLS).

Beyond a certain limit (irreversible exceeding of stress limit), the loss of uncertainty and/or of the syntropic ability of the system determines a local or a widespread reduction in functional freedom (structural and/or functional permanent damage), or a loss of one or more functions, potentially leading to the decline of the biological system, eventually to death (or to a temporary and reversible NDS or NDLS).

It is within this line of research, that we are going to introduce a possible explanation of the so-called Out of Body Experience (OBE) and of the so-called Electromagnetic After-Effect (EAE), leaving at Section 4 the discussion on the so-called Energy Healing Abilities (EHA) and psi phenomena.

\subsection{NDS' out of Body Experience}

I was floating in the very air, rigidly horizontal, a few feet above the bed [...] I

${ }^{41}$ The limit cycle principle (Mitchel J. Feigenbaum) indicates the limit beyond which a dynamical system in a continuous cycle, such as cellular dynamics, can pass (phase transition) from a chaotic state to an ordered state, and vice versa. In the transition between the two states, the strange attractor that governs the dynamics of the system is called a limit cycle attractor.

${ }^{42}(\ldots)$ The stable limit cycle of the original trimolecular two-variable model becomes unstable (and the system diverges) if the velocity constant of the autocatalytic reaction is sufficiently small. If one simply adds a further intermediate variable as a storage substance, the stable limit cycle exhibits a period doubling cascade into chaos when lowering the autocatalytic reaction rate. If the rate is lowered even further, the stable chaotic attractor becomes unstable and the system diverges again. In contrast, in system we observed the same (two-dimensional) behaviour than in the originas two-variable model. Of course the period doubling and the chaotic region in the parameter space could be so narrow that we did not find it numerically, but it seems that the trajectories of the whole three-dimensional model are in fact confined to a two-dimensional surface in the long time limit [97]. 
was moving toward the ceiling, horizontal and powerless [...] I managed to turn around and there [...] was another "me" Lying quietly upon the bed. Sylvan Muldoon, The Projection of the Astral Body, 1929

An OBE [98] [99] [100] can be defined as the presence of the following three phenomenological elements [16]: the feeling of being outside one's physical body (or disembodiment); the presence of a distanced and elevated visuo-spatial perspective (or perspective); and the seeing of one's own body (or autoscopy) from this elevated perspective.

NDErs commonly report the occurrence of OBEs with veridical perception (VP) [101] (VP in NDEs is when a person acquires verifiable information which they could not have obtained by any ordinary means) both locally (i.e., reporting about the immediate surroundings, such as resuscitation efforts) and non-locally (i.e., reporting being in another place, such as describing what relatives were doing and/or saying at a physical distance, as in a waiting room).

As introductory note to the topic at hand, it should be noted thatto qualify experiences such as those we are going to deal with as Out of Body Experience, is specious, since it arbitrarily assigns a spatial collocation to something, namely mind endowed with consciousness, which does not have any spatial collocation. The reason for resorting to the qualification of such experiences as Out of Body Experiences, mostly derives from the religious conditioning exercised on the location and interpretation of phenomena linked to death and dying. As for Western thought, the most emblematic example of such specious approach is given by the Christological approach to the worldwide mythological hierophany of resurrection ${ }^{43}$ :

Between the sixth and the ninth hour of 14 of Nisan, Hebrew year 3791, that is between midday and three o' clock PM of 7 April of 30 D.C. (Christian year), it gets dark over the whole Earth. At the age of 36 - 37 Jesus of Nazareth ${ }^{44}$, the Man ${ }^{43}$ In this regard, see e.g [102].

${ }^{44}$ The traditional date of birth of Christ is $1 \mathrm{AD}$, reconstructed, but incorrectly, by Dionysius the Little (around $523 \mathrm{AD}$ ), and then adopted as the beginning of the Christian era by the monk Bede the Venerable in $725 \mathrm{AD}$. Most historians, however, believe that Christ's birth date should be $4-7$ years earlier than that calculated by Dionysius the Little, going back to 7 - $6 \mathrm{BC}$, that is, before the death of Herod the Great, in accordance with the Gospel narrative according to which the Child is born when Herod is still alive, so as to enable him to order the massacre of the innocents (Matthew 3, 16). Historically, of Herod we know the date of his death, which occurred around 4 BC, a few days before an eclipse of the Moon that manifested itself on the coordinated Palestine, exactly on March 13 of 4 BC. In a chapter of the Gospel of Luke, moreover, we read that Jesus was born during a census ordered by Quirino (future) Governor of Syria, in the year 6 (....), but also this date was the subject of confusion, because Quirino was yes Governor, but in $6 \mathrm{AD}$, and actually took a census that year. Recent studies make it clear that the same Quirino as early as $6 \mathrm{BC}$, when he was not yet Governor but a simple official, made a census, alongside Sanzio Saturnino. Also on the basis of this historical reconstruction the birth of Jesus must be prior or contemporary to the census of $6 \mathrm{BC}$. To conclude, the story about the Nativity tells us only what Matthew writes, when in his Gospel he describes a bright star in the sky. In reality it was not a star but two exceptional events: 1) an astronomical phenomenon that occurs every 854 years, namely the conjunction between Jupiter and Saturn, which by light diffraction becomes five times brighter than the sum of the two bright sources individually; 2) the long-awaited, for that time, the coming of the Three wise-priests-astronomers Kings, of Babylonian origin, the Magi, custodians of the sacred fire of Zarathustra. 
of YHWH ${ }^{45}$, the Logòs who became flesh ${ }^{46}$, emits the Spirit to the Father, putting an end to his earthly essence-existence, surrendering himself to the mystery of a symbol, the Cross ${ }^{47}$ : death and redemption, the ultimate beginning and end, creation and destruction, the Alpha and the Omega. And after Three days He resurrected to Sky.

Soour question is:

Can the consciousness of a dying persondetach from the body, look at it from afar and take a ride around?

At present, "There is general agreement that the seat of consciousness is the brain" [81], meaning that there is general misunderstanding about what con-

${ }^{45}$ Between the $7^{\text {th }}$ and $6^{\text {th }}$ centurie BC, under the siege of the Assyrian Empire, the priestly authority of the Kingdom of Judah, with its capital Jerusalem, for the purpose of coagulating the souls and forces of the Semitic communities (which worshiped different deities), around the common objective of defending themselves from the Assyrian invasion, it made of the Kingdom of Judah the first kingdom in history to venerate in a single temple a single god: the Abramitic god Yahweh, He IS, He who $I S$, divine Sinaitic figure conceived by the Semitic priestly caste during the second half of the $2^{\text {nd }}$ millennium BC (when the Semitic peoples of Sinai invent alphabetic writing), as theological synthesis between the Sumerian cosmic triad Anum-Enlil-Enki (which also includes the female counterpart of Anum, Ki, and the female feminine divinity, Ninhunsag), and the Akkadian one composed by Marduk-Ishtar-Shamash. A divine figure inspired by İ́vara, one of the many names given to Krishna (a divine figure who inspired the hierophany of Chris-t-os, which comes from the ancient Greek chrisos, gold, by interposing the letter T or the Tau of the Egyptian, Phoenician and Hebrew alphabets, symbol of death and resurrection-distinctive characteristics of Krishna-, of the solar cross or light, along with the musical scale $G-A-D$ ), one of the many names, the $57^{\text {th }}$, given to Vishnu, a Vedic masculine divinity, one of the personifications of Tat. According to Genesis (the final drafting of the Book of Genesis, was completed between the $6^{\text {th }}-5^{\text {th }}$ century BC in Judea, by unknown authors), Yahweh gave to man (which in Hebrew is called Adam and corresponds to Sky, while Woman is called Issah and corresponds to Earth) this commandment (Genesis, 2: 16-17): From any tree of the garden you may eat freely; but you must not eat from the tree of the knowledge of good and evil, for when you eat from it you will certainly die. Having he eaten and having survived, so it continues (Genesis, 3: 22): Behold, man has become like one of us in the knowledge of good and evil. Now, lest he reach out his hand and take also of the Tree of Life and eat, and live forever. And Yahweh drove out the male-female couple (Sky-Earth, the primordial antinomy) from Paradise (the absence of antinomies) and in doing so He condemned him to work for living (to obtain artifacts from the rough object) and her to give birth with pain, both to pay with human suffering for the violation of the Divine Law. Implicitly, the punishment imposed recognizes a fact now acquired (Iron Age): technological know-how tied to work, and scientific knowledge linked to knowledge, can change, for better or for worse, the condition of human existence.

${ }^{46}$ The patristic solution, canonized by the Christological Councils, describes in Christ a person with two natures, one divine and one human. Human nature has all the properties (idioms) that distinguish it, and so also the divine one. The will, the strength, the love, the free will, the wisdom, the knowledge, etc., belong to the idioms. Since there are two natures, the human and the divine, in Christ there are two wills (the human and the divine), two forces (the human and the divine) and so on. The idioms belong to the human nature and do not pass from one nature to another, otherwise the two natures would mingle. In Christ, the divine and the human nature are pericorphic, that is, one inside the other, neither mixed nor confused. Christ, having two wills, possesses both the divine and the human will.

${ }^{47}$ The Christian symbolism of the cross is the geometrical transposition of the cosmic spiral decomposed into four cardinal points (quadrature of the circle, historical man), arranged on the horizontal plane (Earth, analytical thought and synthetic thought), and united on the sagittal plane (Axis Mundi) in the central point, the fifth point (whose projection on the circle gives shape to the five-pointed star, whose esoteric meaning will be repeated by the pentagram of the Vitruvian Man by Leonardo da Vinci), in which their projections are joined, the immobile point (the Unmoved Mover of Aristotelian memory) intersected by the vertical axis Zenith-Nadir (Cosmic man, symbolic thought), around which the Spiral of Life and Death orbits. 
sciousness and mind are.

As discussed so far, consciousness, like mind, do not inhabit into the brain or elsewhere. Consciousness is an expression of the state of coherence of the system that supports it, the mind-body correlation system and its mnemopoietic state. This condition could be synthesized by resorting to a metaphorical relationship of equivalence between mind and water, where Mind is a Coherent Domain belonging to the territory of tension, while water is a Coherent Domain belonging to the energy/ matter territory.

- Water is to biological water as mind is to mind endowed with consciousness.

- Water (solid, liquid and gaseous state) responds to the laws of thermodynamics.

- Biological water (super-coherent state of liquid water) responds to quantum electrodynamics laws.

- Mind (neuro-physiological tension state) responds to tension-dynamics laws.

- Mind endowed with consciousness (super-coherent state of mind) responds to psychodynamics of non-equilibrium laws.

As pointed out at paragraph 1, consciousness, and awareness too, does not follow the law of all or nothing, that is to say that its function it can vary with the variation of the state of integrity of the whole correlative system supporting it, which may involve:

1) the state of synergy between poietic/syntropic/mnemotropic action;

2) the correlative dynamics of the poietic function;

3) the correlative dynamics of the syntropic function;

4) the correlative dynamics of the mnemotropic function;

5) the correlative dynamics of the holographic frame that supports the interaction between the functions.

It follows that the typology, degree and extent of 1) a transitory or permanent, reversible or irreversible, altered state of awareness, occurring during an NDS' zeroing state of consciousness, 2) a transitory or permanent, reversible or irreversible, altered state of consciousness, occurring during an NDLS' depressed-distorted state of awareness and consciousness, 3) a transitory or permanent, reversible or irreversible, impaired state of awareness and consciousness, occurring during a state of suspension of awareness and consciousness, such as coma [103], varies with the varying of the cause, the duration and severity of the damage and impairment of the mind-body correlations system, first and foremost those associated to bio-electromagnetic conversion and to energy-tension neuronal transmutation.

Accordingly, during a NDS is not the cognitive state of consciousness to be retrospectively recalled and reconstructed as detached but the psychic $\rightarrow$ mental state of awareness. For there to be a cognitive state of consciousness, there must be available a stream of conscious awareness a subject chooses to focus on when making self-directed efforts to modify and/or modulate the quality and beam of attention. During an NDS this availability is denied, there is no conscious aware- 
ness, no conscious application of effort, no emotional self-regulating strategies available.

During the objective (temporary and reversible) state at the phase boundarybetween life and death, that is during an NDS, the ordinary flow of the relationship of continuity (continuous rhythmic system) implicated in the apparent discontinuity elapsing between the experiencer and the object of her/his experience, between the territory of energy and the territory of tension, between the body dimension and the mind dimension, between her/his inner-insight (embodied) and her/his outer-outsight (embedded), loses its feed-forward dynamics (basin of attraction's self-transformation) to be hanged on a feed-back loop dynamics (almost periodic or periodic). The degree of uncertainty/effectiveness of the syntropic function exerted by the strange attractor's basin of attraction on the dynamics of the whole system is compromised (loss of ability to maintain the rhythmic properties of the system), the process of self-transformation cease at the level of the limit cycle attractor or at the fixed point attractor's limit, with the system's (reversible) exceeding of stress limit, requiring the activation of the available variability of reserve, with a relapse on the processes and/or structures that are under its action.

The selective and discriminative action exerted by the neurological filter and sensory channels are suppressed with the exception of one: the EM sensory module, which continues to be guaranteed by the capacity of biological water to 1) retain electronic charges, in the form of vortical excitations of quasi-free electrons, storable as energetic reserve; 2 ) induce an electronic and protonic long-range and long life excitation of the different molecular species available, enabling their selective activation and mutual attraction; 3) convert mechanical vibrations (phonons) in quantum of electromagnetic energy (photons) and viceversa (piezoelectric effect) [63].

The dissociation (disconnection) state elapsing between bodily (energy) and mental (tension) dimension occurring during an NDS, may induce the subject to experience a (quasi)sensed (not-perceived), floating, unintentional, devoid of space-time coordinates, passive, oneiric-like disembodied and disembedded state of detached awareness (a mind-body regime disengaged from the ordinary Frequency-Phase Correlative Dynamics). Asubmerged, depressed mental state in which 1) all ordinary cognitive functions (including consciousness) are zeroed, 2) the Nervous System tuning (via Phase Conjugate Dynamics; Spin Conjugate Dynamics; Tension Conjugate Dynamics) gives way to the biochemical and biophysical processes that allow the temporary survival of cells, organs and systems in the suppression of neurological activity, 3) while the mind-body system activity it is coupled (on the left-alone subject's neuro-psycho-physiological event-horizon) to Tension-Tension Correlative Dynamics (tensorial interconnection) involving domains of tension coupled to the subject's psychic $\rightarrow$ mental inner-insight and domains of tension coupled to the subject's outer-outsight. In such a state, the subject's egoic psychic complex or self is forced to engage in- 
finding symmetries between body and surrounding, i.e. in finding groundedness coordinates to counteract its drift, only via the EM sensory module, e.g. viaprocesses that could present affinities with (self-) Ghost Imaging reconstruction [104] [105], or (self-) tissues magnetic texture and spin-waves interplay reconstruction (like in holographic spin-wave interference pattern recognition [106] [107]), as to become somehow grounded with respect to the context (this is also the role of the self).

The result of the pre-mortem deprivation of consciousness and ordinary sensory channels, is then the deprivation of the coordinates on which are engaged the symmetries connecting the body with the surrounding, pushing the self adrift and forcing it towards a state of de-symmetrization which is doubled, on the left-alone subject's neuro-psycho-physiological event-horizon, by a Doppler-like effect, in a spatially mirrored reality, where the body is reflecting the mind as a doubledentity.

This reality (quasi) sensed by an adrift-ungrounded self, is retrospectively recalled-reconstructed, through memory processes (retention of the absent) that draw on mnesic material both antecedent and contextual (polarized hysteresis domains $\leftrightarrow$ standing mnesic-like processes reconstruction) to the NDS, as a floating, unintentional, devoid of space-time coordinates, passive, oneiric-like locally veridical perception.

So the answer to the first part of the posed question Can the consciousness of a dying person detach from the body (and) look at it from afar?, is definitely NO, this (as any other) is not the case.

And what about the second part of the same question: and take a ride around?

During an NDS, as stated above, the MBS activity it is coupled (on the left-alone subject's neuro-psycho-physiological event-horizon) to Tension-Tension Correlative Dynamics (tensorial interconnection) involving domains of tension coupled to the subject's psychic $\rightarrow$ mental inner-insight and domains of tension coupled to the subject's outer-outsight. The latter, may include also an outer-outsight spatially afar from the subject, as is the case of a waiting room far from the subject's surrounding, but still reachable and traceable by the correlative dynamics elapsing between EM sensory module's Phase-Tension Correlative Dynamics and Tension-Tension Correlative Dynamics. Retrospectively, this experience is recalled-reconstructed through the same memory processes proposed above, in the form ofnon-locally veridical perception.

Things looks different when not an OBE but, now yes, a (quasi) perceived, floating, intentional, devoid of space-time coordinates, active, oneiric-like disembodied and disembedded state of (altered state of) consciousness, is experienced during a self-induced NDLS.

\subsection{NDLS' out of Body Experience}

Summarizing what stated so far, some basic main differences distinguishing NDLS from NDS can be posed: 
1) during an NDLS the subject is not clinically dead;

2) cognitive functions and consciousness are not zeroed but altered;

3) ordinary sensory channels are not zeroed but altered;

4) ordinary Phase Conjugate Dynamics and Spin Conjugate Dynamics are not (quasi) zeroed but altered;

5) the riddled basin of attraction that governs the mind-body system does not completely lose its ability to self-transform;

6 ) engaging in finding it's coordinates, the self has available, to some extent and in addition to the EM sensory module, also ordinary sensory channels, cognitive functions and a state of consciousness which can provide it with a certain degree, even if distorted, of groundedness, so to avoid getting a drift-ungrounded.

The result is a condition in which an altered state of consciousness is added to a state of distortion of the self, with the subject having available, in some degrees and extent, the possibility to have access toa stream of conscious awareness, to choose to focus on in making self-directed efforts to modify and/or modulate the quality and beam of attention and emotion.

NDLSs can therefore be divided into two groups, based on the possibility, ability and willingness of the subject experiencing it, to make use of the available stream of conscious awareness:

1) a group composed by subject that does have the possibility or ability or willingness to make use of the available stream of conscious awareness, and

2) a group composed by subject that doesn't.

The first group includes all cases of self-induced NDLS, such as self-induced catalepsy (self-hypnotic, voluntary, mediumistic, shamanic), altered states of consciousness induced by deep meditation, or by the intake of psychotropic substances, or by pulmonary hyperventilation, and (eventually) tanatosis or simulated death. The second group includes all the other NDLS cases.All OBEs retrospectively reported by subjects belonging to the latter, can be treated by resorting to the same explanations suggested for OBENDS correlated, with the difference that, for the above outlined reasons, an OBE NDLS correlated refers to a (quasi) perceived, floating, unintentional, devoid of space-time coordinates, passive, oneiric-like disembodied and disembedded state of (altered state of) conscious awareness ( $\rightarrow$ de-symmetrization of the altered state of the self coupled to the altered state of conscious awareness), and not to a (quasi) sensed (not-perceived), floating, unintentional, devoid of space-time coordinates, passive, oneiric-like disembodied and disembedded state of detached awareness as is the case of a OBENDS correlated.

OBEs retrospectively reported by subjects belonging to the first groupdo NOT refer to OBE, i.e. to a de-symmetrized mind-body system experience at the phase boundary between life and death, but to a self-induced suspension of the mind-body correlative dynamics, leading to a Mind-Body Self-Decoupling Experience (MBSDE), i.e. to a (quasi) perceived, floating, intentional, devoid of space-time coordinates, active, oneiric-like disembodied and disembedded state of (altered state of) con- 
sciousness, whose explanation adds some more element to the understanding of mind-body dynamics in states close to the phase limit.

For this purpose, however, it is important to keep in mind that there is nothing of what we can experience through the mind and the body, which is devoid of physical consistency. A gesture, an action, a behavior, a posture, a sound emission, an attitude of the gaze, possess, as much as an object or a physical phenomenon do, a physical consistency that can be traced back to the dynamics of a tension gradient distribution coupled, via Spin Conjugate Dynamics, to the dynamics of an oscillating system. Because of this, they possess their own interferential properties and capacities, which performs on the energy plan. The same thing can be said for a thought or a stream of thoughts (a thought never comes alone), mental images, dreams, feelings, perceptions, intuitions, emotions, memories, psychic functions, psychic complex, conscious and unconscious. They all possess a physical consistency that can be traced backto the dynamics of a tension gradient distribution carryingwhat we experience as neuro-psycho-physiological system (psychodynamics), and possess their own interferential properties and capacities, which performs on the tension plan (Tension Conjugate Dynamics).

As stated in Section 2, each localized (in space and/or in time) form of confinement (tensorial, energetic, massive, subatomic, atomic, supra-atomic, biological, cosmological), i.e. delimited by a boundary, is a tenso-vibrational micro-environment and corresponds to an oscillator or a resonant cavity (cavity resonator), a stationary system organized around a particular tensorial/frequential configuration of perturbations (tensions/oscillations), existing thanks to the relationships of interference it has with the endogenous and exogenous tenso-vibrational environment. That is to say that together, the physicality of the mind and the physicality of the body, form a dynamic, multi-composite and multi-levels system of relationships elapsing between cavity resonators. What is related to the body can be treated as a cavity resonator for energetic phenomena, while what is related to mind can be drawn as a cavity resonator for tensorial phenomena (it must be stressed out that a "cavity resonator for tensorial phenomena" is referred to, as it will be discussed in Section 4, a coherent tension gradients distribution devoid of space-time collocation, i.e. non-localized, whose topological collocation can be traced back, for convenience, to the imaginary space dimension of a four-dimensional space [108] [109] [110], or, as suggested by string theory, of an $n$-dimensional space).

In addition, as we have seen: Phase Conjugate Dynamics and Tension Conjugate Dynamics interfere via Spin Conjugate Dynamics, which means that the interaction between the massive plane "body" and the tensorial plane "mind", takes place thanks to the interface established by quantum dynamics.

What kind of experience is then the one referred to as related to a (quasi) perceived, floating, intentional, devoid of space-time coordinates, active, oneiric-like disembodied and disembedded state of (altered state of) consciousness?

Most likely, during experiences like these what may undergo variations is not 
the (inexistent) mind-body spatial (inside-outside) relationship but the coupling relationship elapsing between the two. That is, mind and body coupling relationship can be temporarily and voluntarily suspended, allowing the mind endowed with consciousness to enter into coupling relationship with other energetic and/or tensorialcavity resonators (since the MBS itself is a cavity resonator, it can in turn be involuntarily hooked, or voluntarily let itself be hooked, by a coupling relationship, possibly manifesting dramatic reactions, as is the case of so-called possession phenomena).

This process, full of pitfalls for the psycho-physical integrity of those who experience it, can be realized only if the mind-body system is voluntarily pushed close to its phase limit (self-induced NDLS). Close to the phase boundary between life and death, normal fish and soluble fish disappear living place to the pond of tension gradient distribution, where instead of separate or quantized entities that interact with each other, there are tensive-images constantly formed and dissolved as in a kaleidoscope, tensive-vortices constantly formed and dissolved as in the current of a river. Vortices and images exist only temporarily and are only a reality to some extent fictitious (virtual) and arbitrary, cut out, remixed and stretched in the turbulent tension gradients floating.

What is eventually experienced in this condition, has nothing to do with the existence of parallel worlds, hidden dimensions, life after death, spirits, ethereal entities and the like (all of this stuff is nothing more but a mind product induced by disembodied and disembedded Tension Conjugate Dynamics).

All that is there, are just the effects of the relationship that is established on a plane of reality (the pond of tension gradient distribution) devoid of spatio-temporal coordinates, namely the subject's neuro-psycho-physiological event-horizon, between the cavity resonator mind endowed with an altered state of consciousness and other cavity resonators coupled to a (or several) living subject's neuro-psycho-physiological event-horizon. That is, any psychic complex dissolved in the neuro-psycho-physiological event-horizon coupled to a living person or community of persons (or even a neuro-psychic complex coupled to a minded or proto-minded neurologic organisms) can interfere with the cavity resonator mind endowed with an altered state of consciousness of the subject experiencing a self-induced NDLS.

The result is a MBSDE, a (quasi) perceived, floating, intentional, devoid of space-time coordinates, active, oneiric-like disembodied and disembedded state of (altered state of) consciousness.

\subsection{NDS-NDLS Electromagnetic After-Effects}

Due to NDS or NDLS the subject who has experienced it can develop a hyper-sensitivity to electromagnetic fields (EHS), a phenomenon known as electro-sensitivity, also called Electromagnetic After-Effect (EAE) [111] [112] [113] [114], which manifests itself as the tendency of the subject to interfere with potentially anything electro-magnetic (natural and/or artificial) in the environment 
(EMEnt). Frequently, EHS is one of a larger complex of sensitivities to which NDSrs (Near Death State experiencers) and NDLSrs (Near Death Like States experiencers) are prone (e.g. allergies, chemical sensitivities, geomagnetic sensitivities, psi phenomena). When this interference relationship is established, both the EAEr (Electromagnetic After Effect Experiencer) and the EMEnt (electro-magnetism of the environement) can be affected.

According to [115] when the interference is established by an apparent action exerted by the EAEr on the EMEnt, we refer to as EM action, inversely, when is established by an apparent action exerted by the EMEnt on the EAEr, werefer to as EM reaction. The deeper the NDS or NDLS it has been, the more likely the NDSer or NDLSer is to report EM actions and reactions, suggesting that the more severe the stressful action the MBS underwent, the deeper are the consequences suffered by cellular or tissue or organ or system's autopoietic processes.

EAErs can report sensing the presence of EMF in different ways (EM reaction), that is visually (e.g. seeing white light, halos, colors becoming blurry), auditorily (e.g. ringing in the ears, humming, crackling and crunching sounds), kinesthetically (e.g. heart poundings, body vibrating, goose bumps, tingling, physical cringes, little shocks across the body, skin crawling, pressure in the forehead, headaches, hairs raising, floating sensations, shaking hands), interoceptively (e.g. nausea, dizziness, lightheadedness, queasiness, pain, sleeplessness, anxiety, agitation, feeling "drained"), olfactorily (e.g. smelling aromas, a burnt smell, a sweet smell, a menthol breath).

Among the possible EM actionsthere are:

- computers and other electronics malfunctioning when near;

- lights dimming, flickering, or burning out when near;

- wrist watch batteries die quickly, or watches malfunctioning when worn.

The contextual onset of EHS in association with other types of sensitivities is manifesting with paresthesia and dysesthesia of various kinds and degrees, and it states for a ground impairment of the mind-body system's adaptive feedforward action-reaction towards environmental stimuli (stressors), calling into question, e.g., the integrity of the Psycho-Neuro-Immunological (PNI) system [116] [117] [118] [119] [120].

It is here assumed that the severely destabilizing conditions at or near the phase boundary between life and death, which goes to meet the subject experiencing an NDS or a NDLS, may vary permanently the phase boundary properties of the barrier that separates the inside from the outside of the whole organism, namely the skin, by varying 1) the dielectric constant of the interfacial water (biological water) coherent/incoherent phase (redox pile) of which skin's cells and layers are perfused, and, as a consequence 2) epidermis, dermis, hypodermis' dielectric constant (dielectric and electrophysical properties, i.e. dielectrophoresis profile), so to develop the EM actions and reactions we observe in EAV (it must be emphasized that we are not among the animals that specialized in the electro-receptor ability). 
Due to the chemical and physical processes that take place as part of normal bodily functions, tiny electrical currents exist within the body, responsible for the emission of a very weak EMF at Extremely Low Frequencies (ELF) and High Frequencies (HF).

So humans do give off electromagnetic radiation. Although the peak of radiation we give off is between 10 to 12 micrometer wavelength, that is in the infrared radiation wavelength (between 0.75 to 1000 micron; 1 micron $=10^{-6}$ meters), we also give off lots of radiation at around $100 \mathrm{GHz}$ ( 1 millimeter wavelength), while normally we don't give off any electric charge ${ }^{48}$.

Electrical currents correlated to cardiac electro-activity and neuro-electro-chemical activity are among the most studied electrical currents existing within the body (Figure 4). Nerves relay signals by transmitting neuro-electro-chemical impulses (soliton waves) down the spinal cord from the brain to the muscles, organs, glands, tissues. Brain waves, having no constant but changing frequency [121], are constantly expanding to the whole nervous system (through the perineural system) and to the whole organism.

The thalamus is a switching and integrating center of neuronal waves coming from sensory system, from the spinal cord and the brain stem to the cerebral cortex. The system is called thalamic rhythmic generator or pacemaker. Calcium ions are seeping slowly to particular thalami-cortical neurons, which are oscillating $1.528 \mathrm{sec}$. and are triggering brain waves. Then, brain waves propagate up to the cerebral cortex. If the targeted neuronal areas are saturated with calcium ions, the thalamic oscillations stop. The brain waves are idling during this silent

${ }^{48}$ Electric and magnetic fields are treated separately in the context of extremely low frequency fields. They have different effects on humans and biological systems. Electric fields and currents are induced inside the body by exposure to extremely low frequency magnetic fields. The fields and currents induced in the body interact with the body's own electric fields and currents, which play an important role in biological functions (e.g. in the conduction of nerve stimuli or in signal transduction at cell membranes via ion currents). Moreover, extremely low frequency electric fields affect electric currents on the body surface. In general, the effects of these fields depend on the strength of the fields, the frequency of the fields, the orientation of the body in the fields and the distance between the human and the field source. With growing distance the fields decrease. External extremely low frequency electric fields are distorted by the presence of a body and create an electric field strength on the body surface by charge transfer, whose strength depends on the size, shape, parts and grounding of the body and its orientation in the field. An electrical surface charging, like e.g. the skin surface and the hairs, is caused like in static fields. Depending on the electric field strengths, these surface effects can be perceptible due to micro-discharges and the movement of hairs, which repel each other and then straighten up. In addition to these surface effects, external extremely low frequency electric fields cause spatially variable charge densities inside the body by the movement of electrical charges (electrostatic induction). The induced electric field inside the body is several hundred thousand to million times weaker than the inducing external $50 \mathrm{~Hz} / 60 \mathrm{~Hz}$ field. Extremely low frequency magnetic fields penetrate the body practically without hindrance. The predominant possible effect is the stimulating effect of the magnetically induced electric fields and body currents (so called eddy currents) inside the tissues (Figure 3). The body's own fields, have current densities of up to $10 \mathrm{~mA} / \mathrm{m}^{2}$. Even higher current densities occur locally in the heart muscle and in the brain. Currents generated by external fields are only perceptible at current densities above $10 \mathrm{~mA} / \mathrm{m}^{2}$ by sensory receptors in the skin or eye and can lead to irritations or impairments in the case of repeated exposure. Acute health risks from nerve, skeletal muscle or heart muscle stimulation are only caused by locally induced fields with current densities of more than $100 \mathrm{~mA} / \mathrm{m}^{2}$. 


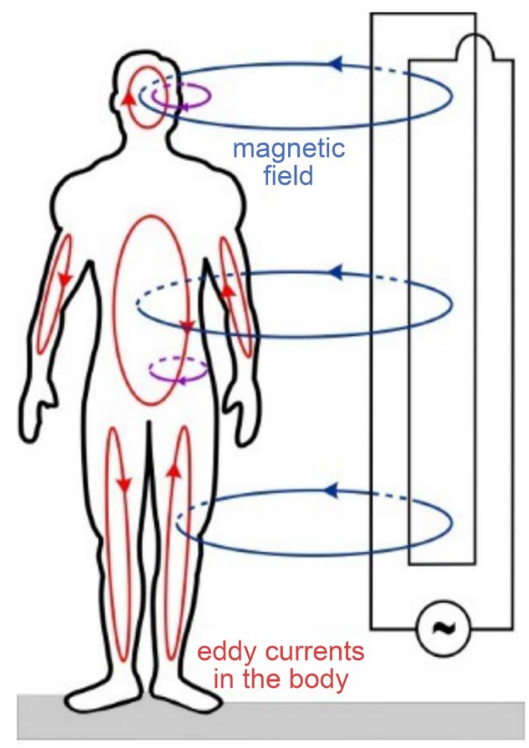

Figure 3. An external extremely low frequency magnetic field causes eddy currents in the human body. The field penetrates the body. The simplified figure shows the eddy currents of an alternating magnetic field perpendicular to the body axis. In healthy conditions the effects of induced fields and currents are not perceptible below the limit values of induced fields and currents in our everyday lives. [Image source: https://www.emf-portal.org/en/cms/page2/home/effects/low-frequency].

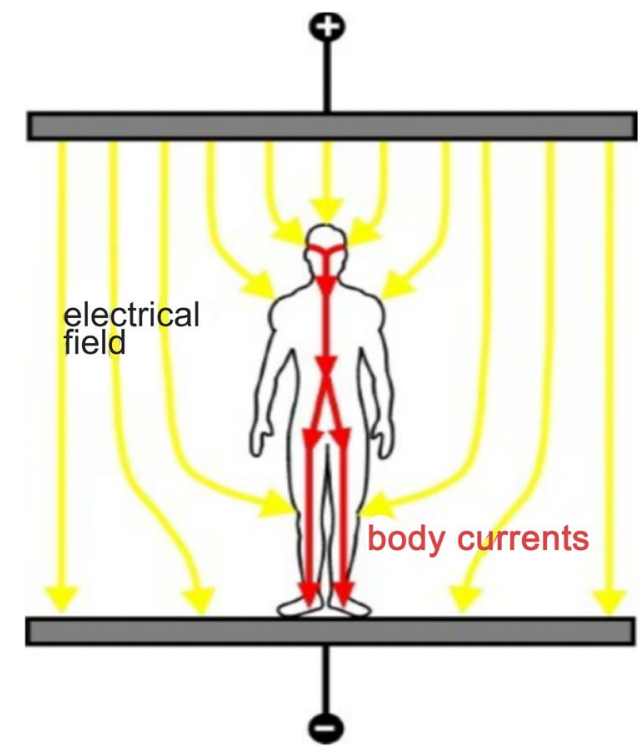

Figure 4. An external electric field causes an electrical charging of the body surface in humans and (in the case of an alternating field) very low internal body currents. The effects of the electric field are therefore generally limited to the body surface. [Image source: https://www.emf-portal.org/en/cms/page2/home/effects/low-frequency].

phase, lasting from 5 to $25 \mathrm{sec}$. The thalamic oscillations start again, when the calcium level in cells drops to the value allowing the neuron to oscillate again. So, the brain waves adjust sensitivity and activity of the whole NS. The time domain where the brain waves are not thalamus controlled is the most interesting 
part of the system. Then the brain field can be affected by electrical and magnetic rhythms from outside, natural or artificial. Geo-electromagnetic earthquake's approaching activity [122] [123] and Schumann resonance disturbance belongs to the former and hypersensitivity to geomagnetic field perturbation, also a possible NDS or NDLS aftereffect, can be triggered by such perturbations. Schumann resonance theory holds that the space between Earth surface and ionosphere behaves as a resonance cavity for terrestrial and extra-terrestrial magnetic and electromagnetic activity. Sun activity (e.g. solar magnetic storms) and thunderbolts, generate electromagnetic standing waves that propagate around the globe, disrupting the ionosphere while Schumann resonance trails off (Figure 5). The effects of these perturbations on the value of the Schumann resonance are felt by all biological systems [87] [124] [125] [126].

In humans, when the PNI system's conditions do not allow to effectively compensate for the effects induced by the perturbation (as it happen in case of hypersensitivity to geomagnetic field perturbation), the organism can manifest one or more adverse reactions.

Electromagnetic field generated in living cells and conditioning biological activity is a nature of life. In eukaryotic cells, the electromagnetic field is generated

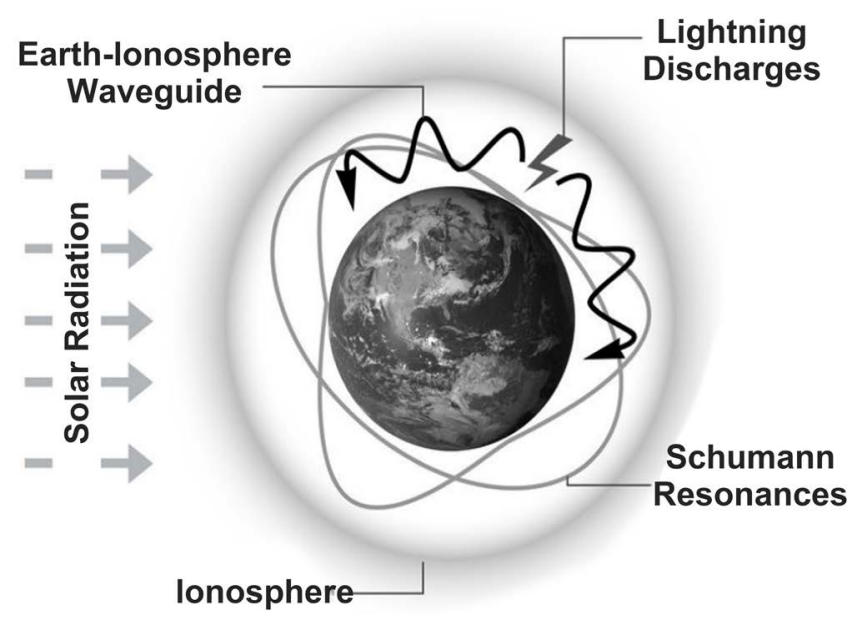

Figure 5. The space in between the surface of the Earth and the ionosphere, an approximately 50- to 375-mile thick layer of non-luminous plasma or charged atmospheric gas, forms an electromagnetic resonant cavity, with a potential resonant frequency range known as the Schumann Pulsed Electro-Magnetic Field Resonances (SPEMFRs). The SPEMFRs [vibrations] exists between the surface of the earth and the ionosphere, which is the border of the magnetosphere. The SPEMFRs are a series of potentiated frequencies, with a baseline of $7.83 \mathrm{~Hz}$, ascending in harmonic progression up the electromagnetic range to $14.3,20.8,27.3$ and $33.8 \mathrm{~Hz}$ (the first four modes). The higher the $\mathrm{Hz}$ value, the shorter the wavelength and the more energy dense the waveform. SPEMFRs occur because the cavity (space) between the surface of the Earth and the ionosphere behaves as a so-called closed wave guide. This means that the waves are behaving as if they stand still within this cavity. This cavity is naturally energized by electric currents which occur during lightning and sun activity. SPEMFRs are a kind of "map" of what is happening in the atmosphere; it is a mathematically derived prediction that says when the atmosphere is struck, it should produce frequencies in a certain range. 
by microtubules composed of tubulin heterodimers with a strong electric dipole. Energy transport, processing, parceling out into small bits and storing into adenosine triphosphate (ATP) and guanosine triphosphate (GTP) form a complex fermentative and oxidative pathway. High-energy electrons are transported down the respiratory chain in the mitochondrial inner membrane, and the released energy is used to pump protons across the inner membrane into the intermembrane space and cytosol. The electrochemical gradient around mitochondria is formed. In functional mitochondria, the actual electric inner membrane potential of the electrochemical gradient is about $-140 \mathrm{mV}$ and the $\mathrm{pH}$ gradient of about $-1 \mathrm{pH}$ unit. Resonant frequencies of microtubules are important parameters for assessment of the cellular functions and interactions with other cells in the tissue. Interaction between cells is mediated by cellular electromagnetic field in the near-infrared range. Microtubules are capable to generate electromagnetic field in a wide spectrum in classical frequency bands up to $20 \mathrm{GHz}$, at $20 \mathrm{THz}$, and in the UV range.

Coherent mechanical vibrations of living cells are measured by atomic force microscopy in the acoustic frequency range. Frequencies of the mechanical vibrations (phonons) and of the electromagnetic field (photons) generated by a cell are equal. External electromagnetic signals can be selectively damped by tissues, according to their being or not in phase with the possible oscillatory motion of the system's components. This specific phase-matching feature operates as a very selective mechanism, a sort of filter discriminating among perturbations and stimuli acting on the system, thus protecting it against any noisy perturbative background or even strong actions, which, however, are out of phase with the oscillatory motions allowed by the system's inner dynamics [127].

Phase-matching means oscillatory resonance. As stated so far, resonance and tension are intrinsic and essential properties the self-organization of the energetic phenomenon. Thanks to them the world can transform itself always remaining similar to itself. But if this is their primary and irreducible role, we would say "causative", their secondary, derived, role is no less important. Resonance and tension act at every level of observation of reality, both physical and psychical. Yet, the biological role of tension is underestimated or not well understood. In the living organism, e.g., every single atom is in an excited state. This is why a constant absorption of energy is required, partly to sustain this aroused state, and partly to transmit energy (radiation) and scatter it in the environment. For a new chain of atoms (i.e., a molecule to emerge), energy is required. In the chain there are free electrons circling, which is what sustains the chain. This only means "required" energy. But the cell also requires the electromagnetic space for the tension inside it to emerge and be preserved. A cell is only able to reproduce itself if it is given energy from the outside because reproduction requires the emergence of tension zones in the cell.

Similarly, the DNA also requires the electromagnetic space for the tension inside it to emerge and be preserved. DNA is only able to reproduce itself if it is 
given energy from the outside because reproduction requires the emergence of tension zones leading to superhelical tension in DNA supercoiling.

Dielectrophoretic attraction of dielectric particles to living cells is observed, and a corresponding frequency of oscillations is assessed in the frequency range 1.5 - $52 \mathrm{MHz}$. Recently, researchers have directed their efforts to distinguish between healthy and pathological state of cells utilizing a technique called dielectrophoresis (DEP), an analytical diagnostic and screening technique that uses the principles of polarization and the motion of bioparticles in applied electric fields [128], that is by detection of cells' dielectric and electrophysical properties ( $d i$ electrophoretic profile) [129] [130], managing to clarify the processes underlying cell membrane impairment due to electrical and mechanical stress (electro-deformation) [131]. DEP is the movement of particles by a trapping force in a non-uniform electric field when the particles and surrounding medium have different polarizabilities. According to the Maxwell-Wagner theory of conductivity in heterogeneous systems, there is a critical frequency that separates the low-frequency range, where ionic conductivity dominates, from the high-frequency range, where dielectric properties determine the system's behavior in the electric field.

Skin layers and different points of the same layer show different conductivity and different dielectric properties. One of the more important electrical properties of the skin is impedance. Electrical impedance is defined as the opposition that show the skin when a current through itself. It is widely accepted that the main electrical impedance resides in the stratum corneum (the uppermost layer of epidermis) while the impedance of the other layers is several orders of magnitudes lower [132].

This resistance is due to the water content of the stratum corneum is very low, not more than $20 \%$, compared to $70 \%$ in the underlying tissue. This means that the skin impedance is dominated by the passive electrical behaviour of the stratum corneum and significant differences in impedance values among different anatomical regions of normal skin have been found. The low frequency pathway is dominated by the appendages such as hair follicles and sweat ducts. Lipid lamellae are borderlines between very low conductivity (lipids) and high conductivity (electrolyte) forming a capacitor. There are two distinguishable pathways involving the lipid layers: a direct pathway through the corneocytes and a tortuous pathway using hydrated sites around the corneocytes. Technically, this it can be modeled as a resistor for the appendages and a resistor-capacitor combination for each capacitive pathway in parallel. Since the parameters of the capacitive pathways are distributed, the number of resistor-capacitor combination should be enormous. This combination system showed by the stratum corneum is very reactive and it shows more impedance than resistance. The skin capacitance is a measure of the charge storage capacity of the skin. Therefore, electroporation is known to dramatically change the electrical resistance of lipid-based barriers, and cell membranes. More recently, electroporation has been suggested 
as being responsible for the rapid and large electrical changes that occur because of "high-voltage" pulsing of tissues.

Skin's biologically active points (BAP; known also as acupuncture points) have much higher electric conductivity, that is the measure of the tissue's ability to conduct an electric current, and their refraction index is different from the points next to them [133].

Comparing with the value registered in ordinary points of the skin, the resistance of BAP is lower and the capacitance is higher, being also not only quantitative, but qualitative differences between the associated impedance spectra. The large dispersion of the in vivo impedance characteristics of the human body is also owed to the distributed presence of BAP.

Chemical bonds (including covalent, ionic, hydrogen and van der Waals types) have been commonly assumed to be dominating for biological organization and activity. However, these bonds represent forces acting at short distances in the $\mathrm{nm}$ region. Biological systems maintain coherence at every dimension scale.

Long-range coherence, large distance cooperation, and the whole body control are significant properties of biological systems.

Electromagnetic field generated in living cells can produce a surplus of water's coherence (coherence between coherent domains) [134].

At charged surfaces, water forms super-coherent layers (Super-Coherent State of Biological Water) with macroscopic thicknesses up to about $500 \mu \mathrm{m}$. The layers are called exclusion zones, as charged particles are excluded from them. Formation of a super-coherent state is related to a transformation mechanism of water molecules. The process is driven by collection of low-grade energy and its transformation into high-grade energy which is able to produce electronic excitations. Energy of thermal fluctuations is transformed into energy of the super-coherent state. The energy of a molecule in the super-coherent state is lower than its energy in the uncoherent state (forming a redox pile, see below). In presence of dysfunctional mitochondria, the surrounding biological water's layer reorganize with a reversed orientation of the electric field, which enables transport of electrons released into cytosol. As biological water occupies $70 \%$ of the cell volume, it is capable of releasing a huge amount of electrons into the cytosol. Free electrons increase conductivity which causes damping of electromagnetic field. The mechanism of damping electromagnetic oscillations generated by microtubules may explain the disturbed organization in cells with dysfunctional mitochondria [135] [136].

As I discussed in [63], QED has introduced the concept according to which the interaction between the vacuum EMF and liquid water induces the formation of large, stable coherent domains (CDs) of about $100 \mathrm{~nm}$ in diameter at ambient conditions, and these CDs may be responsible for all the special properties of water including life itself. The CD is a self-produced cavity for the EMF; the photon of the trapped EMF acquires an imaginary mass, and is therefore unable 
to leave the CD.

According to Giuliano Preparata ${ }^{49}$, Emilio Del Giudice ${ }^{50}$ and colleagues, the water $\mathrm{CD}$ is a quantum superposition of ground coherent state and excited state (in the proportion of 0.87 and 0.13 ). Liquid water is therefore a two-fluid system consisting of a coherent phase (about 40 percent of total volume at room temperature) and an incoherent phase. In the coherent phase, the water molecules oscillate between two electronic configurations in phase with a resonating EMF.

To understand the phenomenological two-phase model of water, the QED approach assumes that the cluster-forming $\mathrm{H} 2 \mathrm{O}$ molecules are in a ground state that differs from the ground state of the isolated molecule. In this new ground state, the $\mathrm{H} 2 \mathrm{O}$ electron cloud is deformed, the effective radius and the dipole moment are increased, so as to generate the hydrogen bonds which stabilize the clusters. In the clusters the water dipoles oscillate coherently, in-phase; the clusters are coherent domains (CDs). Thus pure bulk liquid water consists in two interspersed phases, coherent and incoherent, having widely different dielectric constants (that of the coherent phase is 160 , due to the high polarizability of the coherently aligned water molecules that are oscillating in concert; while the dielectric constant of the incoherent state is about 15). The incoherent phase comprises water molecules in the molecular ground state (as observed in the gas phase) packed in a highly dense state in the interstices around large clusters in which the water molecules perform hindered rotations and interact coherently with a large electromagnetic field.

The externally applied electric fields are therefore only felt in the non-coherent phase. Because coherent water is excited water with a plasma of almost free electrons, it can easily transfer electrons to molecules on its surface. The interface between fully coherent interfacial water and normal bulk water becomes a "redox pile".

Ion-specific effects are widespread, but nowhere are more critically manifested than at the fluid interfaces of biological structures. Action potentials, osmotic flows, energy transduction, and the stabilization of proteins are driven by ion concentration gradients across liquid films on hydrophobic biomaterials. Recent experiments [137] revealed that ions interact specifically at the prototype air-water interface over separations that vastly exceed the range of direct electrostatic forces in any dielectric medium. Such long-range specific ion effects may be triggered by electrostatic and electrodynamic forces, but they must be powered also by other mechanisms, such as the thermal fluctuations intrinsic to fluid interfaces.

In the past, experiments were performed in order to measure the interfacial water index of refraction at optical frequencies and the value measured at the interface was exactly equal to the bulk value. This result is expected when interfacial water is probed by electromagnetic radiation at a wavelength corresponding to the visible region [138].

${ }^{49}$ See: Giuliano Preparata https://en.wikipedia.org/wiki/Giuliano_Preparata

${ }^{50}$ See: Emilio Del Giudice https://en.wikipedia.org/wiki/Emilio_Del_Giudice 
Still, despite a massive amount of literature dedicated to the subject, the dielectric constant of interfacial water and its depth remain essentially unknown because measurements are challenging [139].

The biological water superstructure constitutes a detailed mold of the biomolecule generating molecular electric dipole interactions in the surrounding water environment. The long range dipole correlations issue by the skin's interfacial water are also acting as carrier waves and may "capture" in their coherent oscillations other neighboring molecules, cells, and biological units by phase-matching. Reciprocally, impurities present in the medium or sources of other kinds, external or endogenous, may operate as de-tuning agents, destroying or altering the coherence of the skin's CDs, and in a chain of effects, varying the interfacial water dielectric constant [138] [140] [141], and that of skin layers, and the propagation of the bio-electromagnetic field, with the final result of the appearance of skin pathologies and possibly hypersensitivity to EMF.

Remindingthat the human energy-tension basin of attraction, i.e. the mind-brain-body (de-localized) system, reproduces the fundamental structure characterizing the EEQ-GD, which in turn reproduces the fundamental structure of the ET-GD [45], and thatthe fundamental structure of the EEQ-GD is a unitary fractal and resonant object, a toroidal-like cavity resonator, consisting of an outer frequency weft (Phase Conjugate Dynamics $\rightarrow$ Body), an inner spin weft (Spin Conjugate Dynamics $\rightarrow$ Brain) and a tensorial core (Tension Conjugate Dynamics $\rightarrow$ Mind), we can hypothesize that after an NDS or an NDLS experience, Phase Conjugate Dynamics coupled to Spin Conjugate Dynamics may go under restoring-reorganization, leading toa permanent dielectric constant variation, both at the interfacial water level and at skin's layers level, so to alter the subject's way of emitting and absorbing EMF and develop the EM action and reaction we observe in $\mathrm{EAE}$.

\section{NDS-NDLS Energy Healing Abilities Aftereffect}

EAE is not the only aftereffect that can result from the hypothesized dielectric reorganization. A second one is the possible acquisition of the so-called energy healing abilities (EHA), such as pranotherapy and the like [142], namely the emission by the subject of an EMF at LFs (eventually tested via Gas Discharge Visualization (GDV) Technique [143] [144]) showing healing properties.

In such case, the subject may acquires the possibility to exploit its own long range dipole correlations (or/and to filter, channel and exploit natural electromagnetic oscillations present in the environment (Figure 6)) to "capture" (by phase-matching), in their coherent oscillations, the coherent portion of the perturbed oscillations' spectrum with which it comes to establish an interference relationship, acting on it as a "tuning agent". The possibility to intercept the subtle electromagnetic gradients of a perturbed system's hidden field and to act on it as a "tuning agent" (restoring coherence), is at the basis, e.g., of Chinese acupuncture, Qigong practice [145] [146], Japanese shiatzu, Homeophaty [147] and 


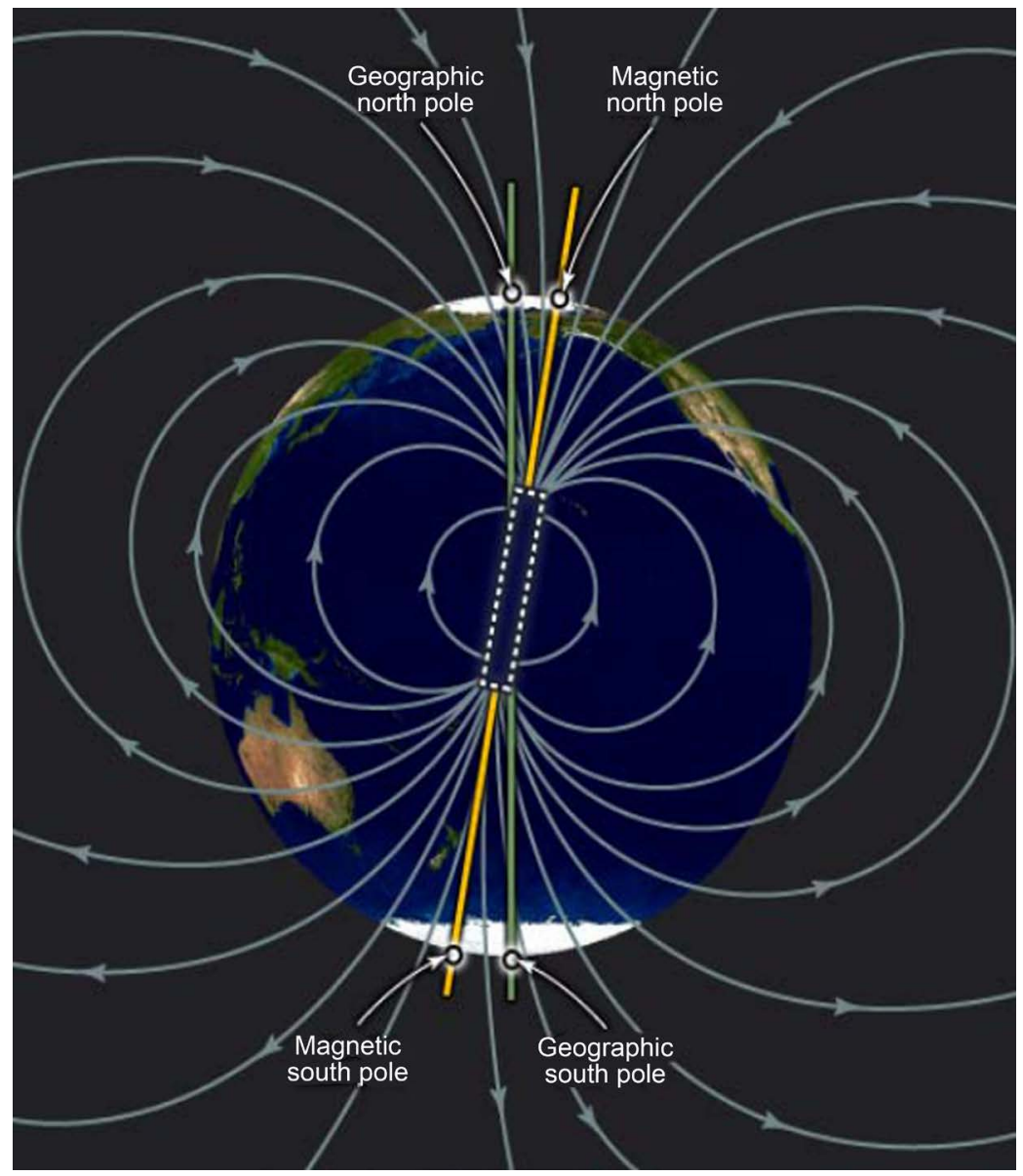

Figure 6. Schematic illustration of the invisible magnetic field lines generated by the Earth, represented as a dipole magnet field. In actuality, our magnetic shield is squeezed in closer to Earth on the Sun-facing side and extremely elongated on the night-side due to the solar wind. Earth's geomagnetic field is trapping a large volume of electrified gas-thus forming the magnetosphere-and forcing the solar wind to flow around it.

of all the apotropaic practices worldwide belonging to ethnomedicine, in the context of magical, exoteric and religious beliefs.

The possibility to intercept and trace the subtle geomagnetic gradients coupled to natural sources, e.g. water and mineral oil, or the subtle electromagnetic gradients coupled to hidden ELF or HF sources, e.g. man made artifacts or biological material,are at the basis of the so-called, respectively, dowsing for water (rhabdomancy) [148] [149] and for things (radiesthesia) [150].

These same possibilities can be effective to disrupt and destabilize a system (see footnote $\mathrm{n}^{\circ} 25$ ). Furthermore, they can be effectivenot only in relation to Phase Conjugate Dynamics of the type Frequency-Phase Correlative Dynamics andto Spin Conjugate Dynamics of the type Phase-Tension Correlative Dynamics, as so far discussed, but alsoin relation to Tension Conjugate Dynamics of the type Tension-Tension Correlative Dynamics.

This latter possibility (which science eventually dismiss as paranormal nonsense) introduces us to the topic of the present ending section. 


\section{NDS-NDLS Psi Phenomena Aftereffect}

The various concepts, too, being illusory, and none of them real, fade away accordingly. Thus, for example, everything postulated of the Whole, the Sangsara and Nirvana, arises from nothing more than mental concepts. Changes in one's train of thought [or one's association of ideas] produce corresponding changes in one's conception of the external world. Therefore, the various views concerning things are merely different mental concepts (....) In general, all things mentally perceived are concepts (....) As soon as one's mind is known to be of the Wisdom of the Voidness, concepts like good and evil Karma cease to exist. Padma-Sambhava, the spiritually endowed Teacher from Urgyan ${ }^{51}$

Apart from OBE, EAE, EHA and the like, possible NDS-NDLS aftereffects are the so-called psi abilities [151] [152], a general term introduced by British parapsychologists R. Thouless and B. P. Wiesner in 1948, that is pointing at all parapsychological phenomena, divisible in two main groups: 1) psychokinesis (the direct influence of mind on matter without any known intermediate physical energy or instrumentation) [153] [154], which includes telekinesis (the ability to move a stationary object without the use of any known physical force), which in turn includes psychic-teleportation (the ability to move an object, called apports, through other physical objects or over great distances) [155], and 2) Extra-Sensory Perceptions (ESPs) [156]-[164], a general term introduced by German parapsychologist Rudolf Tischner (1920) and made popular by American parapsychologist J.B. Rhine (1940), used to denote any manifestations of $p s i$ that appear to be analogous to sensory functions, such as:

a) telepathy (the ability to communicate via mental activity),

b) clairvoyance (the ability to visually perceive things not naturally visible),

c) precognition (the ability to perceive and/or behave in a way that is influenced by a future event that would not be anticipated through any known inferential process),

d) psychometry (the ability to gather information about events or persons from objects that have been handled during the event or handled by the person).

The lecture's abstract entitled Margins of Reality: The Role of Consciousness in the Physical World (from the homonymous book published in 1987), held by psychologist Brenda J. Dunne at the 2004 Lehigh Valley Campus Science Lecture Series (PA), could help us to frame our topic:

Since 1979, the Princeton Engineering Anomalies Research (PEAR) laboratory has been studying the interface of mind and matter through a body of rigorous experiments that address the questions of whether human intention can affect the behavior of random physical devices and systems, and whether human consciousness is able to access information about events that are spatially and temporally remote from its physical location. The answers to both these questions turn out to be surprisingly affirmative, raising profound intellectual and philo${ }^{51}$ Padma-Sambhava teachings, The Knowing of the Mind in Its Self-Identifying, Self-Realizing, Self-Liberating Reality

http://www.holybooks.com/wp-content/uploads/The-Tibetan-Book-of-the-Great-Liberation-Padma -Sambhava.pdf 
sophical challenges to many established scientific assumptions and laying the foundation for a new science of the subjective. How this new science will evolve is still unclear, but there is little doubt that it will require redefinition of such traditional concepts as reproducibility, objectivity, and causality, and will need to find a way to incorporate such intangible and variable features as human desire, expectation, and meaning in its methodology and models.

Scientifically controlled investigations of, and experiments on, psychokinesis (PK) and psychic-Teleportation (p-TP) like those conducted at the Princeton University Engineering Anomalies Research Laboratory by Robert G. Jahn (Dean Emeritus of the School of Engineering) [165], who reported that repeatedly consistent results in mentally affecting material substances has been demonstrated in the lab, have been held in several research centers around the world.

Neverthless, until the last quarter of $20^{\text {th }}$ century many cases that were studied, and the experiments that were performed, were undoubtedly due to fraud or trickery, and only few experiments have occurred under controlled conditions. Most of the credible, scientific (according to Western standards) reports onPK and p-Teleportation phenomenon and related (controlled) experiments have occurred since the end of the $20^{\text {th }}$ century,mostly held in themilitary field, in the USA [166], in the USSR and Russia [167] and in China [168] [169].

From the available scientific literature on the subject, the first emerging data is that the observed PK, p-TP and ESP phenomena, which however infrequent and evasive are documented, do exist and can't be explained by contemporary physics. Not surprisingly, a vast array of scientists and scholars who label (mostly a priori) these phenomena as "magic tricks" will continue to consider them as such, leaving room for exotic and whimsical explanations, which according to cultural orientations and beliefs can call into question the consciousness of matter, divine superpowers, UFOs, ghosts, deceased people, the "all explaining" zero point quantum field and so on and so forth.

Matter is a way of being of energy. Radiation is rarefied energy. Mass is condensed energy. Matter is a combination of the two (an atom is about 99.999999999\% radiation). Radiation and mass are a measure of the energy confinement state ${ }^{52}$. Energy (matter, radiation and mass) is a way of being of tension. Tension is what is left when energy, matter, radiation and mass are taken away, and what is left is a tensorial monopole, a state of non-homogeneous distribution of potential gradients (monopoles) coupled to dynamis (Aristotle), the potency correlated to the action (energheia), efficient cause connected to the motion (where the motion impulse is only prefigured) and its quantitative and qualitative effects, the inherentpotency or intrinsic possibility of a body to be translated into an action (energheia) that may be realized or not, a value of reality only possible with respect to the real action realized (entelecheia) [170].

Tension-dynamis is a value of reality with respect to the real action realized

\footnotetext{
${ }^{52} \mathrm{By}$ confinement state is meant every structuring process of a system of correlations endowed with a degree of subsistence (condition of resonance) such as to make it distinct and/or distinguishable (even when not observable) from the context of the relationships it forms part of.
} 
and viceversa, i.e. the possibility for one and the other to be a value of reality is linked to the contextualization of their relationship: the one's possibility of being is linked to the possibility of being of the other.

Just as water remains water in both the solid, liquid and gaseous state, tension remains tension in both the energy's rarefied and condensed state. The phase transition between one state to the other it occurs via symmetry breaking dynamics. Tension-dynamis is the basin of attraction that governs any energy-matter confinement process. Any energy-matter confinement process is organized around its tension-dynamis basin of attraction, which can be drawn as a cavity resonator for tensorial phenomena, a coherent tension gradients distribution devoid of space-time collocation, i.e. non-localized in space nor in time.

Each rarefied-condensative varieties (electrodynamic varieties, thermodynamic varieties, autopoietic varieties) derives from different correlations among anti-symmetrical/chiral composite quantum states (fermions) and symmetrical/achiral composite quantum states (bosons) on the one hand, and tensorial gradients on the other (tensorial monopole and tensorial dipole), and corresponds to a different coherent oscillatory configuration (domains of oscillatory coherence) that oscillate with a non-linear pattern to the rhythm impressed by a carrier frequency modulation.

Each oscillatory configuration corresponds to a figure of polarized interference in spaceinterfaced (entangled) with a tensorial figure polarized in time (quoting Einstein: Time has no independent existence apart from the order of events by which we measure it [171]), while the transitions between one oscillatory configuration and another correspond to the suppression of certain oscillatory modes or rhythms and certain tensorial gradients, which go from being $e x-$ plicated to implicated, and to the amplification of other oscillatory modes and tensorial gradients that go from being implicated to become explicated.

The hysteresis cycles of the quantum field register, on the quantum event horizon, the behaviour and space-time evolution (spin networks, space-time volumes, tensorial gradients) of the energy-matter systems in the form of inteference figures formed by interferences wave fronts within a certain spectrum of oscillation frequencies.

The interference figures are memorized by the mnemotropic state coupled to the rarefied-condensative variety, which the tension-dynamis basin of attraction/ cavity resonator transfers onto the quantum event-horizon in the form of holographic fractals of spin wave fronts accessible to systems with an isomorphic stereodynamic configuration with respect to the systems that have produced the interference figures ${ }^{53}$.

\footnotetext{
${ }^{53}$ All in-formation of human mind endowed with consciousness is carried, transformed and transmitted as holographic spin-wave interference patterns (carried by phonon and photons interference patterns) on the surfaces of higher order hyperspace fields (neuro-psycho-physiological event-horizon), which are non-locally resonant with the intermediate EM field of the brain. The entire universe, including all the visible and invisible structures within it, is essentially a hologram. According to the fundamental laws of electrodynamics, such in-formation can be transmitted from one fractal-involved field to the other by spin-phase conjugate adaptive resonance.
} 
On the quantum event-horizon, the fractalized wave fronts overlap in multiple dimensions, so that:

- the event-horizon functions as a holographic interface that conserves and transmits in-formation (degrees of freedom of the systems in terms of phase couplings, spin couplings and tension couplings), connecting systems of different dimensions (hyper $\leftrightarrow$ quantum $\leftrightarrow$ middle dimension) and different planes of tension-energy-matter structuring to one another.

The interaction of vessels with the sea surfaceit can be introduced as a metaphor of the above translation process. Fourier showed that any three-dimensional pattern can be analyzed into a set of regular, periodic oscillations that differ only in frequency, amplitude, and phase. Specific waveforms can be exact representations, namely Fourier-transforms, of spatio-temporal objects. When a vessel creates waves on the surface of the sea, it creates Fourier-transforms of its impact on the waters of the sea. It is shown [172] that the surface of the sea is surprisingly information-rich (in-formation, see footnote $\mathrm{n}^{\circ} 23$ ). When its wave-patterns are subjected to mathematical analysis, it discloses in-formation on the passage of ships, e.g. the direction of wind, the effect of shorelines, and other factors. The interfering wave-patterns may be conserved for hours and sometimes for days, after the vessels that created them have passed. Though ultimately they dissipate, eroded by the combined action of gravity, wind, and shorelines, as long as the wave-patterns persist, they provide in-formation on the events that occurred at the sea's surface. But the waves created by vessels on the surface do more than create in-formation regarding their own motion: they also in-form the motion of other vessels. All vessels that traverse the wake that spreads out behind a given vessel are rocked by those waves; in this sense the motion of the wave-creating vessel is translated into the motion of the wave-rocked vessels. The medium that transmits the effects is the surface of the sea: it interconnects the wave-creating with the wave-rocked vessels. And, as all vessels both create waves and are rocked by them, the sea interconnects the motion of all vessels on its surface.

This is what may happen when Phase Conjugate Dynamics and Spin Conjugate Dynamics trace their trajectories in their imaginary spatial dimensions: they leave their Fourier-transforms in their virtual quantum event horizon. Inasmuch as the imaginary spatial dimension interconnects the dynamics of the events that occur in space-time, it functions as a holographic event horizon that encodes the particulars of their becoming and shift them to in-form the becoming of other events.

We may model a mind-event (e.g. a flow of sensations, emotions, feelings, thoughts) in a hyperspace endowed with imaginary spatial dimensions [173] [174], where ordinary spatial dimensions are rotated beyond any intersection with the experiencer spatial frame, and whose time dimension is coincidental with the time dimension of the experiencer. In such $\mathrm{n}$-dimensional hyperspace (namely neuro-psycho-physiological event-horizon), if a tuning coherence is es- 
tablished so that a slight preference is given to one of the mind-events, then a loop between the tuner experiencer and the tuned mind-event is established.

Psi and ESP phenomena, where the intentionality of consciousness acts as a catalyst, are all mediated by Tension-Tension Conjugate Dynamics andcould be treated as follows:

- Psychokinesis. Since a mind-event belongs to the same category of phenomena to which all non-minded tensorial phenomena belong, and since all energetic phenomena possess and are governed by a tensorial core, anyone could, but only a few can, capture in her/his mind's tension-dynamis basin of attraction other's tensorial cores by tension-matching (Tension Conjugate Dynamics), and establish a psi relationship, acting as a psi establisher (PSIEr). After having established a loop with an external object, the tuner can govern (on the quantum event holographic-horizon interface) the relationship that binds the object to its materiality and act on it in various ways.

- Telekinesis. One of these ways consists in being able to reduce, by acting via Spin Conjugate Dynamics of the type Phase-Tension Correlative Dynamicson the correlations among fermions-bosons and tensorial monopole-tensorial dipole, the effect of gravity on the object (or on the PSIEr itself as in self-levitation), so to establish a slight preference to a mind-event which is able tomake movingthe object from one point to another in space.

- Psycho-Teleportation. A second one consists in being able to transferan object (inanimate as a crystal, or animate as an alive insect) from the inside (initial position) to the outside (end position) of a container, making the object disappear when it is still inside, to make it reappear outside (and as for the insect still alive) [155]. In this case, after having established a loop with the external object, the PSIEr 1) reduces (on the quantum event holographic-horizon interface) up to almost zero the material and energetic consistency of the object, 2) reduces up to zero the gravitational action on it, and 3) rotates the ordinary spatial dimensions (via spontaneous breakdown of symmetry, see below) of the object beyond any intersection with the container spatial frame. Having done this, the PSIE restablishes (neuro-psycho-physiological event-horizon's interference patterns) a slight preference to a mind-event which is able totransferthe object in-out without interfering with the walls of the container, and finally reconstructs its energy-matter consistency by following the reverse process.

Spontaneous Breakdownof Symmetry (SBS) it deserve a brief explanation. In QFT it is well known that the ordering of the elementary components of a system is achieved as a result of the SBS and constitutes the observable manifestation of coherence. According to QFT, the dynamics of a system is described by a set of field equations which are postulated to contain all the characteristic features of the system. The fields represent the elementary components of the system, e.g., the EMF, the atomic and molecular system's constituents, their electric charges, and dipole moments, etc. In general, one may consider transformations, 
e.g., rotations, translations, etc., of the fields such that the field equations do not change their form when the fields undergo the said transformations. The dynamics is then said to be invariant under the considered transformations, and these are named symmetry transformations of the dynamics. It may happen that under the action of some external perturbation (in our present case the tuner action exerted by the PSIEr), the state of minimum energy of the system (vacuum), is not symmetric under the symmetry transformations of the dynamics. Then, the symmetry is said to be spontaneously broken. Spontaneously means that the system is driven into the non-symmetric state by its own (internal) dynamics, not forced by the external perturbation which only acts as a trigger. SBS allows the transition from the microscopic scale of the elementary components to the macroscopic scale of the system behavior.

- Telepathy. Telepathy is the most common form of intercorrelation between Tension Conjugate Dynamics in the animal kingdom and is a phylogenetically prescribed way of shifting sensations (and emotions in humans). Telepathy it has nothing to do with mind reading or brain-to-brain messaging (role that is instead erroneously attributed to it by most of the discussions on the subject, see e.g. [175]). What happens between two minded or proto-minded systems during their telepathic correlation has nothing to do with communication, i.e. there is no transmission of information, no semantic content, which can possibly be attributed by association, when there is a sharing of meanings between certain sensations or emotions and their cause. Telepathy is a form of entanglement and occurs on the tensorial plane. The Tension Conjugate Dynamics, belonging to the tension-dynamis basin of attraction/cavity resonator, of two or more subjects can be entangled in a manner that makes it possible for anyone of them to sense-feel in real time what is sensed-felt by anyone of them.

- Clairvoyance. It is a type of correlation in part similar to that of telepathy, except that here the entanglement is not established with a shared mode between two or more subjects. The clairvoyant attempt to establish an entanglement correlation with the tensorial fingerprints of places, events, objects or people far in space or in time. To do so, the PSIEr can resort to divination, an ancient practice adopted in different forms in all cultures throughout the world, which, regardless of the modus operandi adopted, is a way of resorting to an empirically established rhythmic system, filled by psychic tension and subjectively experienced, able to establish an interference relation between different levels of manifestation. Anything that the clairvoyant can seein his/her vision is always conditioned by his/her personal interpretation, cultural beliefs and psychicarrangement.

- Precognition. Anticipatory systems are commune to all biological systems. The ability shown by natural anticipatory systems consists in being able to foresee, with a variable but still significant margin of uncertainty, to which environmental perturbations the biological system could undergo, and be- 
have accordingly. This same ability can be exploited by the PSIEr to make predictions about the future, predictions that however suffer from a margin of uncertainty that is anything but negligible.

- Psychometry. It is a variant of clairvoyance and it occurs similarly.

As discussed in [18] (see also footnote $\mathrm{n}^{\circ}$ ), it can be made a psychodynamic distinction between psychic function, mental function, cognitive function, as follows:

- The psychic function is the relational capacity, distinctive, involved in the phylogenetically prescribed arrangement of the human psychic environment (insight), i.e. its species-specific tensorial basin of attraction (the particular tensorial warp on which it can be weaved the energy weft characterizing the genus Homo) in whom are dissolved all the phase transitions (bifurcations) who went to meet the psychism (C.G. Jung) of the biological phenomenon in the course of phylogeny, passing through the neurological transition up to the bifurcation that led to the emergence of the genus Homo.

- The mental function is the explicated, modal and procedural, form of the psychic function. It refers to the process of in-formation who went to meet the human psychic environment in the relationship with the surrounding. De facto, the psychic function and the mental function have always been compresent (what varies is the prevalence of one over the other), pointing at the Jungian concept of archetype (psychic raw material) and to that of collective unconscious. From the psycho-dynamic point of view, these two functions qualify the constitutive arrangement, and dynamics, of the psychic $\rightarrow$ mental environment which precedes the psychological birth of the genus Homo (Middle Paleolithic).

- The cognitive function, is the relational capacity, gained from the particular differentiation, and subsequent arrangement, of the psychic $\rightarrow$ mental environment, following the psychological birth and, in particular, the development of oral language, together with the maturation of cognitive thinking $(\rightarrow$ bipolar psychic dimension thinking-feeling $\rightarrow$ logical-abstract thinking), to overcome stereotypy, which may be understood as the ability to program significant actions with respect to the ability to formulate meaningful purposes, by resorting to the exercise of ideation and ratiocinative discrimination.

In psychodynamic terms, all the so-called paranormal phenomena develop on the ground of the psychic function coupled to mental function, that is to say on the ground of the "bipolar psychic dimension sensing-intuition", and only secondarily, starting from Middle Paleolithic onward, are mediated by the "bipolar psychic dimension thinking-feeling".

It follows that any attempt to extrapolate these phenomena from their natural psychic $\rightarrow$ mental environment, namely bipolar psychic dimension sensing-intuition, is intended, at best, to generate preachers, charlatans, fetishes and psychopathologies fed by superstitions [176]. Similarly, any attempt to capture these phe- 
nomena in the meshes of the Galilean scientific method is intended, at best, to carry out experiments leading to controversial, uncertain results that will feed the widespread attitude to trivialize, ridicule and manipulate them.

Evidently, the expressions paranormal phenomenon and paranormal activity indicate respectively a phenomenon or an activity that significantly deviates from normality. Now, what should be understood by normality could be summarized by paraphrasing Einstein's cited consideration about time: Normality has no independent existence apart from the order of events by which we measure it. What may be considered normal by, e.g., a "woman of medicine" or by a "women shaman", or by the communities which consider normal to turn to these women in case of need, it is very likely not considered normal by us. Images, oral traditions, and historical descriptions show women as invokers, healers, herbalists, oracles and diviners, ecstatic dancers, shapeshifters, shamanic journeyers, and priestesses of the ancestors. The Chinese $W u$ were ecstatic priestesses who danced to the music of drums and flutes until they reached trance, receiving shen (spirits) into their bodies, healing and prophesying under their inspiration, speaking in tongues, swallowing swords and spitting fire. The power of the shen gathered around the whirling dancers was said to cause objects to rise into the air, to prevent wounds from forming when the dancers slashed themselves with knives. Today's worldwide technology consumersare trying to imitate these invokers, healers, herbalists, oracles and diviners, ecstatic dancers, shapeshifters, shamanic journeyers, and priestesses of the ancestors. The result, most of the time, is pathetic. If it happens that some of them manage to get in touch with the shen power, alleluia, the miracle! If it happens that some of the NDErs or NDLErs, as a consequence of their experience at or close to the phase boundary between life and death, manage to get in touch with paranormal activities, alleluia, the miracle!! What may be "normal", integrated and integrable by an individual and the community in which he/she lives, it can be dangerous, difficult to integrate and hardly integrable by an individual and by an entirely different community. This is the case with paranormal activities experienced or experienceable by a technology consumer that becomes a sci consumer, with or without an NDE or NDLE to tell.

Paranormal phenomena violate the basic principles of Western science, but the hundreds of thousands of humans generations that have migrated on this planet throughout the Paleolithic did not know it, so they integrated their paranormal abilities into their existence, discovering them capable of carrying an adaptive value that allowed the community to be one with its habitat and to survive through the millennia.

\section{Conclusions}

The decisive question for man is: Is he related to something infinite or not? That is the telling question of his life. Only if we know that the thing which truly matters is the infinite can we avoid fixing our interests upon futilities, and upon all 
kinds of goals which are not of real importance (...) If we understand and feel that here in this life we already have a link with the infinite, desires and attitudes change. Carl Gustav Jung ${ }^{54}$

The hypothesis of a life beyond death is a subject that fascinates and torments humanity since human beings began to maintain a relationship of contiguity with the world (psychological birth) [44].

One important thing that is often forgotten or undervalued in dealing with vast and delicate topics like this, it is the need to maintain separate, not to mix, the different logical plans involved, such as the one pertaining to immanence and that pertaining to transcendence, not to mix the different relational modules, such as that pertaining to magical-symbolic thinking and the one pertaining to logical-abstract thinking, not to mix the different categories of thought, rational vs irrational. If we use, as too often happens, these non-interchangeable strategies and perspectives leading to internal representation of external reality, as ingredients to cook a tantalizing soup, at most we will eat a hybrid Truth, half transcendent and half immanent, imbued with quantum physics and exoteric symbols, destined to assume the figure of the man-machine hybrid, the cyborg, as the symbol of an ideal post-gender society, where the engineering and marketing of women's procreative function is declared, by the frenzy of male omnipotence, scientifically outdated, in favour of a hybrid world, declined to the masculine.

Garbage in garbage out say the Anglo-Saxons.

For about a million years, long before the psychological birth of human being took place, the burden of death and the experience of dying were meaningless, i.e. they lacked an interpretation. The advent of the psychological birth changed everything, starting from the relationship with existing. At first, the burden of death and the experience of dying were assimilated to a return to the womb of Great Mother Earth (Mother Goddess Worship). Then, went exorcised in the invocations fed by the fire (libido) of the megalithic sorcerer, wrapped in the haunting aura of the magic word. Then, went suspended and interpreted in the furnace fueled by the Metallurgist Man's fire, immortalized in the folds of written language (carved in letters of fire), leading to aworldwide mythological hierophany: resurrection. Then, reworked and synthesized with the fire of the Techniques of Ecstasy, of Alchemy, of Yoga and the like. Then they changed shape and character in the steam engines, fueled by the fire of Homo Tecnologicus. And finally, were removed in the maze of nuclear reactors and research laboratories, fueled by the will to power of Homo Artificialis.

The current 4.0 version of the Tree of Knowledge of Good and Evil and of the Tree of Life, has its roots in this psychological, historical and cultural heritage. To seize the forces of Nature, to decipher its secrets, was and remains the humus from which it has drawn its vigor.

${ }^{54}$ Cited in: Sandoval, J. M., Knapp, J. C. (2017) Psychology as the Discipline of Interiority, Taylor \& Francis, p. 71. 
At our times, we have to deal with a huge neuro-psycho-marketing operation that attempts to impose a new high-bio-tech body paradigm. The organic spare parts market is expanding (at present interspecies blastocyst complementation offers the possibility of generating human organs in animals ${ }^{55}$ ).

The Positivist Technophilous soul has taken the clear upper hand over his soul mate, the Naturophilous soul [29], who rebels and rises up resorting to some of the arguments (revisited in an all explaining quantum key) that are most dear to it, the paranormal activity and the return to Good Mother Nature.

Are the narratives related to Near Death Experiences in line with this trend?

\section{Conflicts of Interest}

The author declares no conflicts of interest regarding the publication of this paper.

\section{References}

[1] Groth-Marnat, G. (1989) The Near-Death Experience. A Review and Critique. Journal of Humanistic Psychology, 29, 109-133.

http://journals.sagepub.com/doi/abs/10.1177/0022167889291008 https://doi.org/10.1177/0022167889291008

[2] Becker, E. (2010) Birth and Death of Meaning, Chapter IV. Simon and Schuster, Riverside.

[3] Timmermans, S. (1999) Sudden Death and the Myth of CPR, Introduction. Temple University Press, Philadelphia, 6.

http://www.temple.edu/tempress/titles/1388_reg.html

[4] Penny Sartori, R.G.N., et al. (2006) A Prospectively Studied Near-Death Experience with Corroborated Out-of-Body Perceptions and Unexplained Healing. Journal of Near-Death Studies, 25, 69-84.

http://inicia.es/de/luisfountain/archivos/a-prospectively-studied-nde.pdf

[5] Hou, Y., et al. (2013) Infrequent near Death Experiences in Severe Brain Injury Survivors-A Quantitative and Qualitative Study. Annals of Indian Academy of Neurology, 16, 75-81. https://www.ncbi.nlm.nih.gov/pmc/articles/PMC3644787/ https://doi.org/10.4103/0972-2327.107715

[6] Hardham, V. (1996) Embedded and Embodied in the Therapeutic Relationship: Understanding the Therapist's Use of Self Systemically. In: Flaskas, C. and Perlsez, A., Eds., The Therapeutic Relationship in Systemic Therapy, Chapter 4, Karnac, London, 71-89.

https://www.scribd.com/document/128470559/The-Therapeutic-Relationship-in-Sy stemic-Therapy-Systemic-Thinking-and-Practice-Series

[7] Mays, R.G. and Mays, S.B. (2017) NDEs and the Neural Correlates of Consciousness. International Association for Near-Death Studies Conference, Westminster, Colorado.

\footnotetext{
${ }^{55}$ Researchers have successfully grown sheep embryos that contain human cells, a breakthrough that may someday save the lives of many patients who need an organ transplant. Pablo Ross, from the University of California, Davis, and colleagues announced at the 2018 American Association for the Advancement of Science meeting in Texas that they have grown sheep embryos with human cells. Researchers have been using genome editing technique to produce animal embryos that do not develop pancreas. The objective is that human cells introduced to these embryos would grow to replace the missing organ (see e.g. [177]).
} 
https://www.researchgate.net/publication/319875969_NDEs_and_the_Neural_Corr elates_of_Consciousness_-_IANDS_2017_Conference

[8] Greyson, B. (1983) The Near-Death Experience Scale. Construction, reliability, and Validity. The Journal of Nervous and Mental Disease, 171, 369-375.

http://www.newdualism.org/nde-papers/Greyson/Greyson-The\%20Journal\%20of\% 20Nervous\%20and\%20Mental\%20Disease_1983-171-369-375.pdf https://doi.org/10.1097/00005053-198306000-00007

[9] Lange, R., Greyson, B. and Houran, J. (2004) A Rasch Scaling Validation of a 'Core' Near-Death Experience. British Journal of Psychology, 95, 161-177.

http://citeseerx.ist.psu.edu/viewdoc/download?doi=10.1.1.533.9592\&rep=rep1\&type $=\mathrm{pdf}$ https://doi.org/10.1348/000712604773952403

[10] Varela, F. (2002) Consciousness in the Neurosciences-A Conversation of Sergio Benvenuto (1) with Francisco Varela. European Journal of Psychoanalysis, 14. http://www.psychomedia.it/jep/number14/varela.htm

[11] Aspell, J.E. and Blanke, O. (2009) Understanding the Out-of-Body Experience from a Neuroscientific Perspective. In: Murray, C.D., Ed., Psychological Scientific Perspectives on out of Body and Near Death Experiences, Chapter 5, Nova Science Publishers, New York, 73-88.

https://infoscience.epfl.ch/record/154802/files/2009_Aspell(chap)_understanding\% 20the $\% 20$ obe $\% 20$ from $\% 20 \mathrm{a} \% 20$ neuroscientific\%20perspective.pdf

[12] Van Lommel, P. (2006) Near-Death Experience, Consciousness, and the Brain. World Futures, 62, 134-151. http://deanradin.com/evidence/vanLommel2006.pdf https://doi.org/10.1080/02604020500412808

[13] Shemie, S.D., et al. (2014) International Guideline Development for the Determination of Death. Intensive Care Medicine, 40, 788-797.

https://www.ncbi.nlm.nih.gov/pmc/articles/PMC4028548 https://doi.org/10.1007/s00134-014-3242-7

[14] DeGrazia, D. (2017) The Definition of Death. The Stanford Encyclopedia of Philosophy, Edward N. Zalta (ed.). https://plato.stanford.edu/archives/spr2017/entries/death-definition/

[15] Parnia, S., et al. (2014) AWARE-AWAreness during REsuscitation-A Prospective Study. Resuscitation Journal, 85, 1799-1805.

http://www.horizonresearch.org/Uploads/Journal_Resuscitation_2_.pdf https://doi.org/10.1016/j.resuscitation.2014.09.004

[16] Blanke, O. and Mohr, C. (2005) Out-of-Body Experience, Heautoscopy, and Autoscopic Hallucination of Neurological Origin. Implications for Neurocognitive Mechanisms of Corporeal Awareness and Self Consciousness. Brain Research Reviews, 50, 184-199. https://doi.org/10.1016/j.brainresrev.2005.05.008 https://pdfs.semanticscholar.org/4c20/7c40a83847faea92d35a8135f8907fc560f1.pdf

[17] Ward, D. and Stapleton, M. (2012) Es Are Good: Cognition as Enacted, Embodied, Embedded, Affective and Extended. In: Pagliari, F., Ed., Consciousness in Interaction. The Role of the Natural and Social Context in Shaping Consciousness, Advances in Consciousness Research, John Benjamins Publishing Company, Philadelphia, 89-104. https://doi.org/10.1075/aicr.86.06war https://www.academia.edu/648508/Es_are_Good_Cognition_as_Enacted_Embodie d_Embedded_Affective_and_Extended

[18] Messori, C. (2016) Intelligence vs. Artificial Intelligence: The King Is Naked. Open Access Library Journal, 3, 1-37. https://doi.org/10.4236/oalib.1103115 
https://www.scirp.org/Journal/PaperInformation.aspx?PaperID=72003

[19] Kelly, E.W. (2001) Near-Death Experiences with Reports of Meeting Deceased People. Death Studies, 25, 229-249. https://doi.org/10.1080/07481180125967 https://med.virginia.edu/perceptual-studies/wp-content/uploads/sites/267/2015/11/ KEL13-NDEwithReports-of-Meeting-Deceased-People.pdf

[20] SHI TRO, Commentary by Ven. Khenchen Palden Sherab Rinpoche and Khenpo Tsewang Dongyal Rinpoche to the Text by Karma Lingpa, The Profound Dharma of Self-Liberation through the Mind of the Peaceful and Wrathful, Part III, The Bard of Meditation-The Bard of the Moment of Death, Translation by Thupten Nyima. https://www.scribd.com/document/20435024/SHI-TRO-parte-III-Il-bardo-della-me ditazione-e-il-bardo-del-momento-della-morte-I-parte

[21] Van Lommel, P., et al. (2001) Near-Death Experience in Survivors of Cardiac Arrest: A Prospective Study in the Netherlands. The Lancet, 358, 2039-2045. http://www.thelancet.com/journals/lancet/article/PIIS0140-6736(01)07100-8/fulltext https://doi.org/10.1016/S0140-6736(01)07100-8

[22] Pollack, R.A., et al. (2018) Impact of Bystander Automated External Defibrillator Use on Survival and Functional Outcomes in Shockable Observed Public Cardiac Arrests. Circulation, 137, 2104-2113.

http://circ.ahajournals.org/content/early/2018/02/21/CIRCULATIONAHA.117.030 $\underline{700}$ https://doi.org/10.1161/CIRCULATIONAHA.117.030700

[23] Maehle, A.-H. (2014) The Powers of Suggestion: Albert Moll and the Debate on Hypnosis. History of Psychiatry, 25, 3-19. http://journals.sagepub.com/doi/pdf/10.1177/0957154x13500596 https://doi.org/10.1177/0957154X13500596

[24] Ring, K. (1980) Life at Death: A Scientific Investigation of the Near-Death Experience. Coward, McCann \& Geoghenan, New York.

[25] Lutz, A., Greischar, L.L., et al. (2004) Long-Term Meditators Self-Induce High-Amplitude Gamma Synchrony during Mental Practice. The Proceedings of the National Academy of Sciences USA, 101, 16369-16373. https://doi.org/10.1073/pnas.0407401101 https://www.ncbi.nlm.nih.gov/pmc/articles/PMC526201/

[26] Lagopoulos, J., et al. (2009) Increased Theta and Alpha EEG Activity during Nondirective Meditation. The Journal of Alternative and Complementary Medicine, 15, 1187-1192. https://doi.org/10.1089/acm.2009.0113 http://online.liebertpub.com/doi/abs/10.1089/acm.2009.0113?journalCode $=\mathrm{acm}$

[27] Holde, J.M., et al. (2011) Teaching about Near-Death Experiences: The Effectiveness of Using 'The Day I Died'. OMEGA, 63, 373-388.

http://academic.csuohio.edu/kneuendorf/c32117/Holdenetal11.pdf https://doi.org/10.2190/OM.63.4.e

[28] Pasricha, S. (1993) A Systematic Survey of Near-Death Experiences in South India. Journal of Scientific Exploration, 7, 161-171.

https://www.scientificexploration.org/docs/7/jse_07_2_pasricha.pdf

[29] Messori, C. (2017) From Enlightenment to Cyborgs. Open Access Library Journal, 4, 1-46. http://www.oalib.com/articles/5290767\#.WpptIB3OXMw

[30] Tiryakian, E.A. (1972) Toward the Sociology of Esoteric Culture. American Journal of Sociology, 78, 491-512. https://doi.org/10.1086/225361 https://www.jstor.org/stable/2776304?newaccount=true\&read-now=1\&seq=1\#meta data_info_tab_contents

[31] Greyson, B. (2006) Near Death Experiences and Spirituality. Journal of Religion \& 
Science, 41, 393-414. https://doi.org/10.1111/j.1467-9744.2005.00745.x http://spiritualscientific.com/yahoo_site_admin/assets/docs/GreysonNDEandSpirit uality.79194349.pdf

[32] SHI TRO, Commentary by Ven. Khenchen Palden Sherab Rinpoche and Khenpo Tsewang Dongyal Rinpoche to the text by Karma Lingpa, The Profound Dharma of self-liberation through the Mind of the Peaceful and Wrathful, Part IV, Translation by Thupten Nyima.

http://it.scribd.com/doc/29441669/SHI-TRO-parte-IV-Il-bardo-del-momento-della -morte-II\%C2\%B0-parte

[33] Persinger, M.A. and St-Pierre, L.S. (2011) The Biophysics at Death: Three Hypotheses with Potential Application to Paranormal Phenomena. NeuroQuantology, 9, 36-40. https://www.neuroquantology.com/index.php/journal/article/view/388 https://doi.org/10.14704/nq.2011.9.1.388

[34] Bòkkon, I. and Salari, V. (2012) Hypothesis about Brilliant Lights by Bioluminescent Photons in Near Death Experiences. Medical Hypoteses, 79, 47-49.

https://www.ncbi.nlm.nih.gov/pubmed/22543076 https://doi.org/10.1016/j.mehy.2012.03.028

[35] Dotta, B.T., et al. (2011) Photon Emissions from Human Brain and Cell Culture Exposed to Distally Rotating Magnetic Fields Shared by Separate Light-Stimulated Brains and Cells. Brain Research, 1388, 77-88.

http://www.5mp.eu/fajlok/bokkon-brain-imagery/persinger_2011_brain_res_www. 5mp.eu_.pdf https://doi.org/10.1016/j.brainres.2011.03.001

[36] Hu, H. and Wu, M. (2011) Important Photon Emission Results Reported by Michael Persinger's Group. Journal of Consciousness Exploration \& Research, 2, 1555-1556. http://file://C:/Users/eeePC/Downloads/192-592-1-PB.pdf

[37] Primas, H. (2009) Complementary of Mind and Matter. In: Primas, H. and Atmanspacher, H., Eds., Recasting Reality, Springer, Berlin, 178.

https://www.researchgate.net/publication/252490173_Recasting_Reality https://doi.org/10.1007/978-3-540-85198-1_9

[38] Pollack, R. (2015) Eugenics Lurk in the Shadow of CRISPR. Science, 348, 871. http://www.columbia.edu/cu/biology/pdf-files/faculty/pollack/2015\%20Pollack\%20 R.\%20_CRISPR\%20eugenics_\%20Science.pdf https://doi.org/10.1126/science.348.6237.871-a

[39] Goldenfeld, N., Kadanoff, L. P. (1999) Simple Lessons from Complexity. Science, 284, 87-89. http://guava.physics.uiuc.edu/ nigel/articles/complexity.html https://doi.org/10.1126/science.284.5411.87

[40] Witze, A. (2018) Signs of Buried Lake on Mars Tantalize Scientists. Nature, 560, 13-14. https://doi.org/10.1038/d41586-018-05795-6 https://www.nature.com/magazine-assets/d41586-018-05795-6/d41586-018-05795-6 .pdf

[41] Tartèse, R., et al. (2018) Insights into the Origin of Carbonaceous Chondrite Organics from Their Triple Oxygen Isotope Composition. Proceedings of the National Academy of Sciences, 115, 1-5. https://doi.org/10.1073/pnas.1808101115 https://www.researchgate.net/publication/326843842_Insights_into_the_origin_of_ carbonaceous_chondrite_organics_from_their_triple_oxygen_isotope_composition

[42] Rosenblum, B. and Kuttner, F. (2000) The Observer in the Quantum Experiment. https://arxiv.org/ftp/quant-ph/papers/0011/0011086.pdf

[43] Schwartz, J.M., Stapp, H.P. and Beauregard, M. (2005) Quantum Physics in Neu- 
roscience and Psychology: A Neurophysical Model of Mind-Brain Interaction. Philosophical Transactions of the Royal Society B, 360, 1309-1327.

https://arxiv.org/ftp/q-bio/papers/0401/0401019.pdf https://doi.org/10.1098/rstb.2004.1598

[44] Messori, C. (2016) From Continuity to Contiguity. On the Genesis of Consciousness, Culture and Oral Language (Part I-II-III-IV). Journal of Consciousness EXploration \& Research, 7, 163-228.

https://www.researchgate.net/publication/295857703_From_Continuity_to_Contig uity_On_the_genesis_of_consciousness_culture_and_oral_language_Part_I_of_IV

[45] Messori, C. (2012) A Cosmogonic Model of Human Consciousness: Part I-II-III-IV. Journal of Consciousness Exploration \& Research, 3, 1149-1208.

https://www.researchgate.net/publication/255696726_A_Cosmogonic_Model_of_H uman_Consciousness

[46] Messori, C. (2000) Il Sole e la Luna. Sulla Natura dei Simboli e della Mente Umana. Federico Ceratti Editore, Milan, Italy.

[47] Messori, C. (2004) Le Metamorfosi della Meraviglia. Riflessioni sui Percorsi della Conoscenza dall'Età del Bronzo ad Oggi. Maremmi Editori Firenze Libri, Florence, Italy.

[48] Messori, C. (2011) Cells, Neurons, and Qualia: The Holographic Strange Attractor Model. Journal of Consciousness Exploration and Research, 2, 1417-1437. https://www.researchgate.net/publication/255696668_Cells_Neurons_and_Qualia_ The_Holographic_Strange_Attractor_Model

[49] Messori, C. (2012) Dalla Facoltà Acustico-Musicale alle Origini del Linguaggio Orale Fino al Predominio della Cavità Orale che Genera il Mondo sulla Cavità Uterina che Genera la Vita. Il Minotauro, Persiani Editore, Bologna, 2, 6-43.

[50] de Sá-Nogueira Saraiva, R. (2006) Classic Ethology Reappraised. Behavior and Philosophy, 34, 89-107. http://behavior.org/resources/184.pdf

[51] de Sá-Nogueira Saraiva, R. (2010) Evolutionary Psychology of the I/Me and the Idea of the Immortal Soul. Revista Portuguesa de Filosofia, 66, 945-962. http://static1.1.sqspcdn.com/static/f/811206/21057453/1353682695240/Evolutionary +Psychology.pdf?token=o\%2FBPxfwbz5zuBG\%2BBp5zO9LYe4KM\%3D

[52] Ilya Prigogine, I. (1981) From Being to Becoming: Time and Complexity in the Physical Sciences. W. H. Freeman \& Co., New York.

[53] Di Corpo, U. and Vannini, A. (2012) Syntropy: The Energy of Life. Kindle Edition.

[54] Vannini, A. (2005) Entropy and Syntropy. From Mechanical to Life Science. NeuroQuantology Journal, 3, 88-110.

https://www.neuroquantology.com/index.php/journal/article/view/64/64

[55] Maturana, H.R. and Poerksen, B. (2004) From Being to Doing: The Origins of the Biology of Cognition. Carl Auer, Heidelberg.

[56] Maturana, H.R., Mpodozis, J. and Letelier, J.C. (1995) Brain, Language, and the Origin of Human Mental Functions. Biological Research, 28, 15-26.

[57] Rosen, R. (1978) Fundamentals of Measurement and Representation of Natural Systems. North Holland, New York.

[58] Rosen, R. (1991) Life Itself: A Comprehensive Inquiry into the Nature, Origin, and Fabrication of Life. Columbia University Press, Columbia.

[59] Rosen, R. (2012) Anticipatory Sistems. Philosophical, Mathematical and Methodological Foundations. 2nd Edition, Springer, Berlin.

[60] King, C.C. (1989) Dual-Time supercausality. Physics Essays, 2, 128-151. 
https://pdfs.semanticscholar.org/c91b/930170ccda8e63aab2e4790fe6d27291553d.pdf https://doi.org/10.4006/1.3035859

[61] King, C.C. (1996) Fractal Neurodyamics and Quantum Chaos: Resolving the Mind-Brain Paradox through Novel Biophysics. In: Fractals of Brain Fractals of Mind, Advances in Consciousness Research 7, John Benjamins Publishing Company, Amsterdam, 179-234.

https://pdfs.semanticscholar.org/8238/e016a219eb04b5eb08e2db9cb74d8672eb0d.p df

[62] Varela, F.J., Thompson, E. and Rosch, E. (1992) The Embodied Mind: Cognitive Science and Human Experience. The MIT Press, Cambridge.

https://monoskop.org/images/b/b2/Varela_Thompson_Rosch_-_The_Embodied_M ind_Cognitive_Science_and_Human_Experience.pdf

[63] Messori, C. (2018) The Super-Coherent State of Biological Water. Labirinto d'Acque 2018-International Conference and Exhibition, Parma, 21-24 March 2018. https://www.researchgate.net/publication/326225414_The_super-coherent_state_of biological_water

[64] Jung, C.G. (2015) Collected Works of C. G. Jung. Routledge, Abingdon-on-Thames.

[65] Huelga, S.F. and Plenio, M.B. (2013) Vibrations, Quanta and Biology. Contemporary Physics, 54, 181-207. https://arxiv.org/abs/1307.3530 https://doi.org/10.1080/00405000.2013.829687

[66] Streltsov, A., et al. (2015) Measuring Quantum Coherence with Entanglement. Physical Review Letters, 115, 1-8. https://arxiv.org/pdf/1502.05876.pdf

[67] Collini, E., et al. (2010) Coherently Wired Light-Harvesting in Photosynthetic Marine Algae at Ambient Temperature. Nature, 463, 644-647. https://doi.org/10.1038/nature08811

[68] Engel, G.S., et al. (2007) Evidence for Wavelike Energy Transfer through Quantum Coherence in Photosynthetic Systems. Nature, 446, 782-786.

https://doi.org/10.1038/nature05678

[69] Peters, J.F., Tozzi, A. and Eva, D. (2017) Towards Equations for Brain Dynamics and the Concept of Extended Connectome. SciFed Journal of Neuroscience, 1, 1-9. http://www.scifed.com/open-access/towards-equations-for-brain-dynamics-and-the -concept-of-extended-connectome.pdf

[70] Faure, P. and Korn, H. (2001) Is There Chaos in the Brain? Concepts of Nonlinear Dynamics and Methods of Investigation. Life Sciences, 324, 773-793. http://fge.if.usp.br/ reynaldo/verao/caos2.pdf

[71] Freeman, W.J. and Vitiello, G. (2015) Matter and Mind Are Entangled in EEG Amplitude Modulation and Its Double. University California, Berkeley, USA.

[72] Aihara, K. (1997) Chaos in Neural Networks. In: Grebogi, C. and Yorke, J.A., Eds., The Impact of Chaos on Science and Society, United Nations University Press, Tokyo, $394 \mathrm{p}$.

http://www.nzdl.org/gsdlmod?e=d-00000-00---off-0cdl--00-0----0-10-0---0---0direc t-10---4------0-11--11-en-50---20-about---00-0-1-00-0-0-11-1-0utfZz-8-00-0-0-1110-0utfZz-8-00\&a $=\mathrm{d} \& \mathrm{c}=\mathrm{cdl} \& \mathrm{cl}=\mathrm{CL} 4.178 \& \mathrm{~d}=\mathrm{HASH} 014 \mathrm{~b} 485 \mathrm{~d} 6 \mathrm{~b} 46632 \mathrm{c} 9 \mathrm{aaa} 4 \mathrm{~d} 26.7 \#$ HASH014b485d6b46632c9aaa4d26.7

[73] Freeman, W.J. and Vitiello, G. (2010) Vortices in the Brain Waves. International Journal of Modern Physics B, 24, 3269-3295. https://arxiv.org/pdf/0802.3854.pdf

[74] Zerroug, A., Terrissa, L. and Faure A. (2013) Chaotic Dynamical Behavior of Recurrent Neural Network. Annual Review of Chaos Theory. Bifurcations and Dynamical Systems, 4, 55-66. http://arctbds.com/volume4/arctbds_submission_26.pdf 
[75] Rosen, R. (1985) Anticipatory Sistems. Philosophical, Mathematical and Methodological Foundations. 2nd Edition, Springer, Berlin.

[76] Viana, R.L., et al. (2009) Riddled Basins in Complex Physical and Biological Systems. Journal of Computational Interdisciplinary Sciences, 1, 73-82. https://doi.org/10.6062/jcis.2009.01.02.0007

[77] Ott, E., et al. (1994) The Transition to Chaotic Attractors with Riddled Basins. Physica D: Nonlinear Phenomena, 76, 384-410. https://doi.org/10.1016/0167-2789(94)90047-7

[78] Bohm, D. (1996) Universo m.ente material. [Wholeness and the Implicate Order.] Red Edizioni, Italy, 11-16.

[79] Tozzi, A., Peters, J.F., et al. (2017) Topodynamics of Metastable Brains. Physics of Life Review, 21, 1-20. https://doi.org/10.1016/j.plrev.2017.03.001 https://www.sciencedirect.com/science/article/pii/S1571064517300520?via\%3Dihub

[80] Thornburg, J. (2007) Event and Apparent Horizon Finders for $3+1$ Numerical Relativity. Living Reviews in Relativity, 10, 3. http://arxiv.org/pdf/gr-qc/0512169v2.pdf https://doi.org/10.12942/lrr-2007-3

[81] Adamski, A.G. (2011) Bioplasma Concept of Consciousness. NeuroQuantology, 9, 681-691. https://doi.org/10.14704/nq.2011.9.4.382

http://citeseerx.ist.psu.edu/viewdoc/download?doi=10.1.1.841.4586\&rep=rep1\&type $=\mathrm{pdf}$

[82] Haas, A.S. (2011) The Nature of the Relation between Psychology and Physics: An Argument for a Central Role of Electromagnetism in Thought and Behavior. NeuroQuantology, 9, 892-909. https://doi.org/10.14704/nq.2011.9.4.487 https://pdfs.semanticscholar.org/e098/f0082be5250df78dfcf078811e13a90abc67.pdf

[83] Belova, N.A. and Acosta-Avlos, D. (2015) The Effect of Extremely Low Frequency Alternating Magnetic Field on the Behavior of Animals in the Presence of the Geomagnetic Field. Journal of Biophysics, 2015, Article ID: 423838. https://www.hindawi.com/journals/jbp/2015/423838/

[84] Fels, D. (2016) Physical Non-Contact Communication between Microscopic Aquatic Species: Novel Experimental Evidences for an Interspecies Information Exchange. Journal of Biophysics, 2016, Article ID: 7406356. https://www.hindawi.com/journals/jbp/2016/7406356/ https://doi.org/10.1155/2016/7406356

[85] Vanderstraeten, J. and Gillis, P. (2010) Theoretical Evaluation of Magnetoreception of Power-Frequency Fields. Bioelectromagnetics, 31, 371-379.

https://docslide.net/documents/theoretical-evaluation-of-magnetoreception-of-pow er-frequency-fields.html https://doi.org/10.1002/bem.20568

[86] Zhadin, M.N. (2001) Review of Russian Literature on Biological Action of DC and Low-Frequency AC Magnetic Fields. Bioelectromagnetics, 22, 27-45.

https://onlinelibrary.wiley.com/doi/abs/10.1002/1521-186X\%28200101\%2922\%3A1 \%3C27\%3A\%3AAID-BEM4\%3E3.0.CO\%3B2-2\# https://doi.org/10.1002/1521-186X(200101)22:1<27::AID-BEM4>3.0.CO;2-2

[87] Galland, P. and Pazur, A. (2005) Magnetoreception in Plants. Journal of Plant Research, 118, 371-89. https://doi.org/10.1007/s10265-005-0246-y https://www.readbyqxmd.com/read/16283069/magnetoreception-in-plants

[88] Puthoff, H. and Targ, R. (1974) Psychic Research and Modern Physics. In: Mitchell, E.D. and White, J., Eds., Psychic Exploration: A Challenge for Science, G. P. Putnam's \& Sons, New York, 536-538. 
[89] Amoroso, R.L. (1996) The Production of Fröhlich and Bose-Einstein Coherent States in Vitro Paracrystaline Oligomers Using Phase Control Laser Interferometry. Bioelectrochemistry and Bioenergetics, 41, 39-42.

https://www.researchgate.net/publication/228914684_The_production_of_Frohlich _and_Bose-Einstein_coherent_states_in_in_vitro_paracrystalline_oligomers_using phase_control_laser_interferometry https://doi.org/10.1016/0302-4598(96)01925-3

[90] Reimers J.R., et al. (2009) Weak, Strong, and Coherent Regimes of Fröhlich Condensation and Their Applications to Terahertz Medicine and Quantum Consciousness. PNAS, 106, 4219-4224. http://www.pnas.org/content/106/11/4219

[91] Preto, J. (2012) Semi-Classical Statistical Approach to Fröhlich Condensation Theory. arXiv: 1203.2006.

[92] Mesquita, M.V., Vasconcellos, A.R. and Luzzi, R. (2004) Considerations on Undistorted-Progressive X-Waves and Davydov Solitons, Fröhlich-Bose-Einstein Condensation, and Cherenkov-Like Effect. Biosystems Brazilian Journal of Physics, 34, 489-503. http://www.sbfisica.org.br/bjp/files/v34_489.pdf

[93] Banerjee, A., et al. (2015) Proximate Kitaev Quantum Spin Liquid Behaviour in $\alpha$-RuCl3. Nature Materials, 15, 733-740. https://arxiv.org/abs/1504.08037

[94] Wessels, P., et al. (2016) Direct Observation of Isolated Damon-Eshbach and backward Volume Spin-Wave Packets in Ferromagnetic Microstripes. Scientific Reports, 6, Article ID: 22117. https://www.nature.com/articles/srep22117

[95] Brizhik, L.S., Musumeci, F. and Ho, M.-W. (2003) Energy and Information Transfer in Biological Systems: How Physics Could Enrich Biological Understanding. Proceedings of the International Workshop. Acireale, Catania, Italy.

[96] Dulbecco, R. (1950) Experiments on Photoreactivation of Bacteriophages Inactivated with Ultraviolet Radiation. Journal of Bacteriology, 59, 329-347. https://jb.asm.org/content/jb/59/3/329.full.pdf

[97] Wilhelm, T. and Hanggi, P. (1999) What Can Be Stated by the Glansdorff-Prigogine Criterion Concerning the Stability of Mass-Action Kinetic Systems? Journal of Chemical Physics, 110, 6128-6134. https://pdfs.semanticscholar.org/2805/88ec818194b1350e9fcf70fdb0fad2964769.pdf

[98] Rousseau, D. (2011) Near-Death Experiences and the Mind-Body Relationship: A SystemsTheoretical Perspective. Journal of Near-Death Studies, 29, 399-435. https://pdfs.semanticscholar.org/4c47/1c5a6c722ce4728a48153cdb87f25195e751.pdf

[99] Fracasso, C. and Friedman, H. (2011) Near-Death Experiences and the Possibility of Disembodied Consciousness. NeuroQuantology, 9, 41-53.

https://www.google.it/url?sa=t\&rct=j\&q=\&esrc=s\&source=web\&cd=13\&ved=0ahUKEwjAht rU0NnZAhVBzaQKHf6FCQ44ChAWCDcwAg\&url=http\%3A\%2F\%2Fneuroquantology.c om\%2Findex.php\%2Fjournal\%2Farticle\%2Fdownload\%2F389\%2F378\%2526h\%253DHAQ FDYrTn\&usg=AOvVaw2vNM8cE7a8N8XogPzbeU2U https://doi.org/10.14704/nq.2011.9.1.389

[100] Sartori, P., Badham, P. and Fenwick, P. (2006) A Prospectively Studied Near-Death Experience with Corroborated Out-of-Body Perceptions and Unexplained Healing. Journal of Near-Death Studies, 25, 69-84. http://inicia.es/de/luisfountain/archivos/a-prospectively-studied-nde.pdf

[101] Mays, R.G. and Mays, S.B. (2015) The Importance of Veridical Perceptions and Veridical Information in Near-Death Experiences. International Association for Near-Death Studies Conference, San Antonio, Texas. https://www.researchgate.net/publication/285270551_The_Importance_of_Veridica 
1_Perceptions_and_Veridical_Information_in_Near-Death_Experiences

[102] Martini, C.M. (1980) Il Problema storico della risurrezione negli studi recent. [The Historical Problem of Resurrection in Recent Studies.] Gregorian Biblical BookShop, Analecta Gregoriana, 104, 32.

[103] Auxéméry, Y. (2013) The "Near-Death Experience" during Comas: Psychotraumatic Suffering or the Taming of Reality? Medical Hypotheses, 81, 379-382. http://www.medical-hypotheses.com/article/S0306-9877(13)00250-8/fulltext

[104] Zhu, R., et al. (2018) Spatial Multiplexing Reconstruction for Fourier-Transform Ghost Imaging via Sparsity Constraints. Optics Express, 26, 2181-2190. http://proxy.osapublishing.org/DirectPDFAccess/6A92418B-C645-DE81-37CF76C3 C2971DD4_380954/oe-26-3-2181.pdf?da $=1 \& \mathrm{id}=380954 \&$ seq $=0 \&$ mobile $=$ no https://doi.org/10.1364/OE.26.002181

[105] Zhang, Y., et al. (2016) A Novel Compressed Sensing Method for Magnetic Resonance Imaging: Exponential Wavelet Iterative Shrinkage-Thresholding Algorithm with Random Shift. International Journal of Biomedical Imaging, 2016, Article ID: 9416435. https://www.hindawi.com/journals/ijbi/2016/9416435/

[106] Carvalho-Santos, V.L., Elías, R.G. and Nunez, A.S. (2015) Spin Wave Vortex from the Scattering on Bloch Point Solitons. Annals of Physics, 363, 364-370.

http://repositorio.uchile.cl/bitstream/handle/2250/136368/Spin-wave-vortex.pdf;seq uence $=1$ https://doi.org/10.1016/j.aop.2015.10.005

[107] Gertz, F., et al. (2014) Magnonic Holographic Memory. IEEE Transactions on Magnetics, 51, 1-12. https://doi.org/10.1109/TMAG.2014.2362723

http://citeseerx.ist.psu.edu/viewdoc/download?doi=10.1.1.896.668\&rep=rep1\&type $=\mathrm{pdf}$

[108] Tozzi, A., Peters, J.F. and Jaušovec, N. (2018) EEG Dynamics on Hyperbolic Manifolds. Neuroscience Letters, 683, 138-143.

https://www.researchgate.net/publication/323343253_THE_MANIFOLD_OF_LIFE _THE_MATH_SUBTENDING_LIVING_BEINGS'_DYNAMICS https://doi.org/10.1016/j.neulet.2018.07.035

[109] Tozzi, A. (2016) Borsuk-Ulam Theorem Extended to Hyperbolic Spaces. In: Peters, J.F., Ed., Computational Proximity. Excursions in the Topology of Digital Images, Springer-Verlag, Berlin, 169-171.

[110] Tozzi, A. and Peters, J.F. (2016) Towards a Fourth Spatial Dimension of Brain Activity. Cognitive Neurodynamics, 10, 189-199. https://doi.org/10.1007/s11571-016-9379-z

[111] Nouri, F.M., et al. (2008) Electromagnetic After-Effects of Near-Death Experiences. Journal of Near-Death Studies, 27, 83-110.

https://digital.library.unt.edu/ark:/67531/metadc799170/m2/1/high_res_d/vol27-no 2-83.pdf

[112] Nouri, F.M. (2008) Electromagnetic Aftereffects of Near-Death Experiences. https://digital.library.unt.edu/ark:/67531/metadc9054/m2/1/high_res_d/dissertation .pdf

[113] Fracasso, C. and Friedman, H. (2012) Electromagnetic Aftereffects of Near-Death Experiences: A Preliminary Report on a Series of Studies Currently Under Way. Journal of Transpersonal Research, 4, 34-55.

https://www.researchgate.net/profile/Cheryl_Fracasso/publication/278965261_Electromagnet ic_Aftereffects_of_Near-Death_Experiences_A_Preliminary_Report_on_a_Series_of_Studies _Currently_Underway/links/5588412008ae8c4f340639c2/Electromagnetic-Aftereffects-of-Ne 
ar-Death-Experiences-A-Preliminary-Report-on-a-Series-of-Studies-Currently-Underway.pd $\mathrm{f}$

[114] Greyson, B., et al. (2015) Electromagnetic Phenomena Reported by Near-Death Experiencers. Journal of Near-Death Studies, 33, 213-243. https://pdfs.semanticscholar.org/6c57/8065533b927d4e609718de9a2572299bc817.p df

[115] Blalock, S., et al. (2016) Electromagnetic and Other Environmental Effects Following Near-Death Experiences: A Primer. Journal of Near-Death Studies, 33, 181-211.

[116] Ziemssen, T. and Kern, S. (2007) Psychoneuroimmunology-Cross-Talk between the Immune and Nervous Systems. Journal of Neurology, 254, II/8-II/11. https://www.researchgate.net/publication/6329468_Psychoneuroimmunology_-_Cr oss-talk_between_the_immune_and_nervous_systems https://doi.org/10.1007/s00415-007-2003-8

[117] Tausk, F., et al. (2008) Psychoneuroimmunology. Dermatologic Therapy, 21, 22-31. http://www.google.it/url?q=http://onlinelibrary.wiley.com/doi/10.1111/j.1529-8019. 2008.00166.x/pdf\&sa=U\&ved=0ahUKEwjorfqDs8DdAhWRuIsKHTjLBLMQFgglM Ak\&usg=AOvVaw0HSntxHMFXzYHmBvLGeS2M https://doi.org/10.1111/j.1529-8019.2008.00166.x

[118] Honeyman, J.F. (2016) Psychoneuroimmunology and the Skin. Acta Dermato-Venereologica, 217, 38-46.

http://www.google.it/url?q=https://www.medicaljournals.se/acta/content_files/dow nload.php\%3Fdoi\%3D10.2340/00015555-2376\&sa=U\&ved=0ahUKEwjorfqDs8DdA hWRuIsKHTjLBLMQFggSMAE\&usg=AOvVaw2VOIKDYjpedj-hqQzIok0b

[119] Hillert, L. (2001) Hypersensitivity to Electricity; Symptoms, Risk Factors and Therapeutic Interventions. Karolinska University Press, Sweden.

http://citeseerx.ist.psu.edu/viewdoc/download?doi=10.1.1.1000.5868\&rep=rep1\&ty pe $=$ pdf

[120] Tseng, M.-C.M., et al. (2011) Prevalence and Psychiatric Comorbidity of Self-Reported Electromagnetic Field Sensitivity in Taiwan: A Population-Based Study. Journal of the Formosan Medical Association, 110, 634-641.

https://core.ac.uk/download/pdf/82734017.pdf https://doi.org/10.1016/j.jfma.2011.08.005

[121] Tozzi, A. and Peters, J.F. (2016) Brain Low- and High-Frequencies Are Linked through Power Laws and Solidstate Physics. Technical Report.

https://www.researchgate.net/publication/293651250_Brain_low-_and_high-_frequ encies_linked_through_power_laws_and_solid-state_physics

[122] Freund, F. and Stolc, V. (2013) Nature of Pre-Earthquake Phenomena and their Effects on Living Organisms. Animals, 3, 513-531.

http://www.mdpi.com/2076-2615/3/2/513 https://doi.org/10.3390/ani3020513

[123] St-Laurent, F., et al. (2006) Earthquake Lights and the Stress-Activation of Positive Hole Charge Carriers in Rocks. Physics and Chemistry of the Earth Parts $A / B / C, 31$, 305-312. https://doi.org/10.1016/j.pce.2006.02.003

https://www.researchgate.net/publication/223675852_Earthquake_lights_and_the_s tress-activation_of_positive_hole_charge_carriers_in_rocks

[124] Peñuelas, J., et al. (2004) Diamagnetic Susceptibility and Root GrowthResponses to Magnetic Fields in Lens Culinaris, Glycine Soja, and Triticum Aestivum. Electromagnetic Biology and Medicine Journal, 23, 97-112.

http://www.creaf.uab.es/global-ecology/Pdfs_UEG/penuelas-ebm-Diamagneticsusce ptibilityr.pdf 
https://doi.org/10.1081/LEBM-200032772

[125] Kondrachuk', A.V. and Hasenstein, K.H. (2001) The Effects of HGMFs on the Plant Gravisensing System. Advances in Space Research, 27, 1000-1005.

https://www.academia.edu/26681046/The_effects_of_HGMFs_on_the_plant_gravis ensing_system

https://doi.org/10.1016/S0273-1177(01)00167-3

[126] Pazur, A., Schimek, C. and Galland, P. (2007) Magnetoreception in Microorganisms and Fungi. Central European Journal of Biology, 2, 597-659.

https://www.researchgate.net/publication/215658422_Magnetoreception_in_microo rganisms_and_fungi

[127] Brizhik, L., et al. (2018) Modeling Meridians within the Quantum Field Theory. Journal of Acupuncture and Meridian Studies, In Press, 8 p.

https://www.jams-kpi.com/article/S2005-2901(18)30076-1/pdf

[128] Rahman, N.A., et al. (2017) Dielectrophoresis for Biomedical Sciences Applications: A Review. Sensors (Basel), 17, 449. https://doi.org/10.3390/s17030449 https://www.ncbi.nlm.nih.gov/pmc/articles/PMC5375735/

[129] Pethig, R., et al. (2010) Dielectrophoresis: A Review of Applications for Stem Cell Research. Journal of Biomedicine and Biotechnology, 2010, Article ID: 182581. https://www.researchgate.net/publication/44618612_Dielectrophoresis_A_Review_ of_Applications_for_Stem_Cell_Research https://doi.org/10.1155/2010/182581

[130] Dukhin, A.S., et al. (2014) Peculiarities of Live Cells' Interaction with Micro- and Nanoparticles. In: Colloid and Interface Science in Pharmaceutical Research and Development, Elsevier, Amsterdam, 193-222. https://doi.org/10.1016/B978-0-444-62614-1.00010-7

[131] Du, E., et al. (2018) Erythrocyte Membrane Failure by Electromechanical Stress. Applied Sciences, 8, 174. https://doi.org/10.3390/app8020174 https://www.ncbi.nlm.nih.gov/pmc/articles/PMC5909407/

[132] Lu, F., et al. (2018) Review of Stratum Corneum Impedance Measurement in Non-Invasive Penetration Application. Biosensors, 8, 31. https://www.ncbi.nlm.nih.gov/pmc/articles/PMC6023082/ https://doi.org/10.3390/bios8020031

[133] Salceanu, A., et al. (2013) Approaches on Measurements of Human Skin Electrical Resistance. Buletinul Institutului Politehnic Din Iasi, LIX (LXIII), 67-78. http://www.bulipi-eee.tuiasi.ro/archive/2013/fasc.4/p6_f4_2013.pdf

[134] Tedeschi, A. (2010) Is the Living Dynamics Able to Change the Properties of Water? International Journal of Design \& Nature Ecodynamics, 5, 60-67. https://www.witpress.com/Secure/ejournals//papers/D\&NE050108f.pdf

[135] Pokorný, J., et al. (2017) Warburg Effect-Damping of Electromagnetic Oscillations. Electromagnetic Biology and Medicine, 36, 270-278.

https://emmind.net/openpapers_repos/Endogenous_Fields-Mind/General/EM_Can cer/2017_Warburg_effect_damping_of_electromagnetic_oscillations.pdf https://doi.org/10.1080/15368378.2017.1326933

[136] Pokorný, J. (2014) Cancer-Pathological Breakdown of Coherent Energy States. Biophysical Reviews and Letters, 9, 115-133.

https://www.researchgate.net/profile/Jan_Vrba/publication/263805231_CANCER-PAthologi cal_breakdown_of_coherent_energy_states/links/57207e6308aed056fa236c4d.pdf?disableCov erPage $=$ true https://doi.org/10.1142/S1793048013300077 
[137] Enami, S. and Colussi, A.J. (2013) Long-Range Specific Ion-Ion Interactions in Hydrogen-Bonded Liquid Films. The Journal of Chemical Physics, 138, Article ID: 184706.

https://pdfs.semanticscholar.org/1917/fadacf2b318da1d31b95c489483dd33ab30d.pd $\mathrm{f}$

[138] Teschke, O., et al. (2001) Interfacial Water Dielectric-Permittivity-Profile Measurements Using Atomic Force Microscopy. Physical Review E, 64, Article ID: 011605.

http://repositorio.unicamp.br/bitstream/REPOSIP/60423/1/WOS000169907100061. pdf

[139] Fumagalli, L., et al. (2018) Anomalously Low Dielectric Constant of Confined Water. Science, 360, 1339-1342. https://doi.org/10.1126/science.aat4191 https://arxiv.org/ftp/arxiv/papers/1806/1806.04486.pdf

[140] Bonthuis, D.J., et al. (2015) Interfacial Layer Effects on Surface Capacitances and Electro-Osmosis in Electrolytes. Philosophical Transactions of the Royal Society, 374, Article ID: 20150033. http://rsta.royalsocietypublishing.org/content/374/2060/20150033

[141] Bonthuis, D.J. (2014) Dielectric Profiles and Ion-Specific Effects at Aqueous Interfaces. Electrostatics of Soft and Disordered Matter, 129-142.

http://www-thphys.physics.ox.ac.uk/people/DouweBonthuis/documents/2014_Bont huis_bookchapter.pdf https://doi.org/10.1201/b15597-13

[142] Moga, M.M. (2014) Magnetic Field Activity during Psychic Healing: A Preliminary Study with Healing Touch Practitioners. Journal of Nonlocality, III, 1-23.

http://journals.sfu.ca/jnonlocality/index.php/jnonlocality/article/view/50/53

[143] Korotkov, K.G., et al. (2010) Application of Electrophoton Capture (EPC) Analysis Based on Gas Discharge Visualization (GDV) Technique in Medicine: A Systematic Review. The Journal of Alternative and Complementary Medicine, 16, 13-25. https://pdfs.semanticscholar.org/719b/b390a011237007a897142b1c37930e2d37c5.p df https://doi.org/10.1089/acm.2008.0285

[144] Kostyuk, N., et al. (2011) Gas Discharge Visualization: An Imaging and Modeling Tool for Medical Biometrics. International Journal of Biomedical Imaging, 5, Article ID: 196460. http://www.hindawi.com/journals/ijbi/2011/196460/

[145] Yan, X., et al. (2002) Certain Physical Manifestation and Effects of External Qi of Yan Xin Life Science Technology. Journal of Scientific Exploration, 16, 381-411. http://citeseerx.ist.psu.edu/viewdoc/download?doi=10.1.1.486.3229\&rep=rep1\&type $=\mathrm{pdf}$

[146] Yan, X., et al. (1999) Structure and Property Changes in Certain Materials Influenced by the External Qi of Qigong. Material Research Innovations, 2, 349-359. http://yanxinqigong.cn/papers/MRI90020349.pdf https://doi.org/10.1007/s100190050113

[147] Sagi, M. (2016) The New Homeophaty: A New Paradigm in Information Medicine. World Futures, 72, 1-16.

https://dokumen.tips/documents/information-medicine-the-new-homeopathy-a-ne w-paradigm-in-the-new-homeopathy.html

[148] Betz, H.-D. (1995) Unconventional Water Detection: Field Test of the Dowsing Technique in Dry Zones: Part 1. Journal of Scientific Exploration, 9, 1-43. http://citeseerx.ist.psu.edu/viewdoc/download?doi=10.1.1.458.6149\&rep=rep1\&type $=\mathrm{pdf}$ 
[149] Betz, H.-D. (1995) Unconventional Water Detection: Field Test of the Dowsing Technique in Dry Zones: Part 2. Journal of Scientific Exploration, 9, 159-189. http://citeseerx.ist.psu.edu/viewdoc/download?doi=10.1.1.558.6201\&rep=rep1\&type $=\mathrm{pdf}$

[150] Krick, E. (1955) Entropy Logic Theory and Expanding Horizons of Consciousness. In: Nesterov, V.I., Ed., Physical Basics of Informational Interaction: Collection of Articles, 31-41.

https://www.scancoaching.nl/wp-content/uploads/2014/10/Physical\%20Basics\%20o f\%20Informational\%20Interaction\%20pub.pdf

[151] Parker, A. and Brusewitz, G. (2003) A Compendium of the Evidence for Psi. European Journal of Parapsychology, 18, 33-52. https://psy.gu.se/digitalAssets/1338/1338645_parker--brusewitz-ejp-.pdf

[152] Helfrich, W. (2017) Statistical Parapsychology as Seen by an Applied Physicist. Journal of Scientific Exploration, 31, 387-418. https://www.scientificexploration.org/docs/31/jse_31_3_Helfrich.pdf

[153] Bosch, H. and Steinkamp, F. (2006) Examining Psychokinesis: The Interaction of Human Intention with Random Number Generators-A Meta-Analysis. Psychological Bulletin, 132, 497-523. https://doi.org/10.1037/0033-2909.132.4.497 https://pdfs.semanticscholar.org/62aa/e31a291f3cd08925cb4cafed1e355a0e83af.pdf

[154] Schmidt, H. (1982) Collapse of the State Vector and Psychokinetic Effect. Foundations of Physics, 12, 565-581. https://doi.org/10.1007/BF00731929 https://www.researchgate.net/publication/226735437_Collapse_of_the_state_vector _and_psychokinetic_effect

[155] Davis, E.W. (2004) Teleportation Physics Study. Special Technical Report for the Air Force Research Laboratory (AFRL)/Space and Missile Propulsion Division, Propellant Branch (PRSP), 1-78. https://doi.org/10.21236/ADA425545 https://www.researchgate.net/publication/235083700_Teleportation_Physics_Study

[156] Radin, D.I. and Nelson, R.D. (1989) Evidence for Consciousness-Related Anomalies in Random Physical Systems. Foundations of Physics, 19, 1499-1514. http://ww.w.leyline.org/papers/pdf/REG.meta-analysis.1989.pdf https://doi.org/10.1007/BF00732509

[157] Vernon, D.J. (2017) Exploring Precall Using Arousing Images and Utilising an On-LIine Memory Recall Practice Task. Journal of the Society for Psychical Research, 81, 66-79. https://www.spr.ac.uk/sites/spr.ac.uk/files/publications/JSPR\%2081.2.pdf

[158] Storm, L. and Tressoldi, P.E. (2017) Gathering in More Sheep and Goats: A Meta-Analysis of Forced-Choise Sheep-Goat ESP Studies, 1994-2015. Journal of the Society for Psychical Research, 81, 79-107. https://www.spr.ac.uk/sites/spr.ac.uk/files/publications/JSPR\%2081.2.pdf

[159] Baker, I.S., et al. (2017) A Controlled Study of Psychometry Using Psychic and Non-Psychic Claimants with Actual and False Readings Using a Mixed-Methods Approach. Journal of the Society for Psychical Research, 81, 108-122. https://www.spr.ac.uk/sites/spr.ac.uk/files/publications/JSPR\%2081.2.pdf

[160] Becker, C.B. (1990) Extrasensory Perception, Near-Death Experiences, and the Limits of Scientific Knowledge. Journal of Near-Death Studies, 9, 11-20. http://freedownloadb.com/pdf/bextrasensory-perception-b-near-death-experiencesand-the-33342326.html https://doi.org/10.1007/BF01074097

[161] Phillips, S.M. (1995) Extrasensory Perception of Subatomic Particles. Historical 
Evidence. Journal of Scientific Exploration, 9, 489-525.

http://freedownloadb.com/pdf/bextrasensory-perception-b-of-subatomic-particles-i -historical-4949678.html

[162] Tressoldi, P.E., Storm, L. and Radin, D. (2010) Extrasensory Perception and Quantum Models of Cognition. NeuroQuantology Journal, 8, 581-587.

[163] Laszlo, E. (2009) In Defense of Intuition: Exploring the Physical Foundations of Spontaneous Apprehension. Journal of Scientific Exploration, 23, 51-58.

http://citeseerx.ist.psu.edu/viewdoc/download?doi=10.1.1.596.9689\&rep=rep1\&type $=\mathrm{pdf}$

[164] Puthoff, H.E. and Targ, R. (1976) A Perceptual Channel for Information Transfer over Kilometer Distances: Historical Perspective and Recent Research. Proceedings of the Institute of Electronics and Radioengineers IEEE, 64, 329-354.

http://citeseerx.ist.psu.edu/viewdoc/download?doi=10.1.1.447.6775\&rep=rep1\&type $=\mathrm{pdf}$ https://doi.org/10.1109/PROC.1976.10113

[165] Dunne, B.J. and Jahn, R.G. (1995) Consciousness and Anomalous Physical Phenomena, Technical Note PEAR 95004. Princeton Engineering Anomalies Research, Princeton University, School of Engineering/Applied Science. https://www.greaterreality.com/pear/finalcap.pdf

[166] Hubbard, G.S. and May, E.C. (1982) Countermeasures: A Survey and Evaluation (U). Final Report for DIA, 1-19.

https://www.cia.gov/library/readingroom/docs/CIA-RDP96-00788R001300260001$\underline{0 . p d f}$

[167] Kembach, S. (2013) Unconventional Research in USSR and Russia: Short Overview. 1-23. https://arxiv.org/pdf/1312.1148.pdf

[168] Hin, O.K., Liang, S.H. and Choon, T.S. (2013) Information-Energy Equivalence in Qigong: Reviewing Dossey and Schwartz's “Therapeutic Intent/Healing Bibliography of Research" in Light of Pang Ming's Three Levels Theory of Matter. http://journals.sfu.ca/jnonlocality/public/journals/1/PREPRINTS/KeanII1020212.p df

[169] Zha, D. (1990) Parapsychology in the People's Republic of China: 1979-1989. Report for the CIA, 1-17.

https://www.cia.gov/library/readingroom/docs/CIA-RDP96-00789R002600290003$\underline{0 . p d f}$

[170] Sgarbi, M. (2012) Translatio Studiorum: Ancient, Medieval and Modern Bearers of Intellectual History. Brill, Leiden, 61-63.

[171] Barnett, L. (1948) The Universe and Dr. Einstein. William Sloane Associate, New York, 12.

[172] Pethiyagoda, R., et al. (2018) Time-Frequency Analysis of Ship Wave Patterns in Shallow Water: Modelling and Experiments. Ocean Engineering, 158, 123-131. https://arxiv.org/pdf/1702.06275.pdf https://doi.org/10.1016/j.oceaneng.2018.01.108

[173] Carter, P.J. (2014) Imaginary Physics. http://vixra.org/pdf/1210.0124v3.pdf

[174] Carter, P.J. (2014) Consciousness and Perception in Higher-Dimensional Quantum Space-Time. NeuroQuantology, 12, 46-75.

https://neuroquantology.com/index.php/journal/article/viewFile/724/639 https://doi.org/10.14704/nq.2014.12.1.724

[175] Grau, C., et al. (2014) Conscious Brain-to-Brain Communication in Humans Using Non-Invasive Technologies. PLoS One, 9, e105225. 
https://www.ncbi.nlm.nih.gov/pmc/articles/PMC4138179/ https://doi.org/10.1371/journal.pone.0105225

[176] Adebayo, S.O. and Ilori, O.S. (2013) Influence of Paranormal Beliefs on Psychopathology in a Cross-Cultural Society. IOSR Journal of Humanities and Social Science, 11, 63-70. https://doi.org/10.9790/0837-1126370

https://www.researchgate.net/publication/236963973_Influence_of_Paranormal_bel iefs_on_Psychopathology_in_a_Cross-Cultural_Society

[177] Francipane, M.G. and Lagasse, E. (2016) Towards Organs on Demand: Breakthroughs and Challenges in Models of Organogenesis. Current Pathobiology Reports, 4, 77-85. https://www.ncbi.nlm.nih.gov/pmc/articles/PMC5624548/ 